\title{
Diagonal and Off-Diagonal Anharmonicity in Hydrogen-Bonded Systems
}

\author{
Dissertation \\ zur Erlangung des mathematisch-naturwissenschaftlichen Doktorgrades \\ „Doctor rerum naturalium“ \\ der Georg-August-Universität Göttingen
}

im Promotionsprogramm Chemie

der Georg-August University School of Science (GAUSS)

vorgelegt von

Matthias Heger

aus Kiel

Göttingen, 2016 


\section{Betreuungsausschuss:}

Prof. Dr. Martin A. Suhm, Institut für Physikalische Chemie

Prof. Dr. Ricardo A. Mata, Institut für Physikalische Chemie

\section{Referent:}

Prof. Dr. Martin A. Suhm

\section{Korreferent:}

Prof. Dr. Ricardo A. Mata

\section{Weitere Mitglieder der Prüfungskommission:}

Prof. Dr. Burkhard Geil, Institut für Physikalische Chemie

Prof. Dr. Alec Wodtke, Institut für Physikalische Chemie

Prof. Dr. Konrad Koszinowski,

Institut für Organische und Biomolekulare Chemie

PD Dr. Thomas Zeuch, Institut für Physikalische Chemie

Datum der mündlichen Prüfung: 20. April 2016 
If your flight is going remarkably well, something was forgotten.

(Pilot wisdom) 



\section{Acknowledgment}

It is a popular notion that "no man is an island"; and of course, neither am I. Without the help and support by those around me, it would have been outright impossible for me to come this far in my life, and I would like to express my gratitude to the people who have accompanied and instructed me throughout the past years. First and foremost, my family and friends have been at my side through so many difficult times that I cannot even begin to describe how thankful I am for them; and I am deeply moved by the unconditional love and support that my parents have given me.

Martin Suhm and Ricardo Mata have been the most central figures to my scientific upbringing, and I would like to thank them for guiding me through the challenges of our joint theoretical and practical work. This further extends to Anja Poblotzki and Jonas Altnöder, for driving the methanol-anisole project; Jannik Mechau, for helping with the ethene measurements during his internship; and in general, all current and past members of the Suhm Group-especially Franz Kollipost, Katharina Otto, and Julia Zischangfor all the discussions, extended coffee breaks, and whatever else I annoyed them with. Hannes Gottschalk is assuming responsibility for the filet jet setup now, and I am sure that it will be in good hands with him. Beyond my colleagues in Göttingen, the fruitful collaboration with René Wugt Larsen and Jonas Andersen has played an important role for my work, and my short stay with them has spawned some valuable matrix spectra and scientific insights into the methanol dimer (let alone tuna pizza and frozen pastries).

Most of our research would be impossible without the staff of the institute and department workshops, and I would like to acknowledge the support of Volker Meyer, Reinhard Hildebrandt, Frank Rustemeier, Bernd Wichmann, Andreas Knorr and Annika von Roden. And I cannot go without an honorable mention of Werner Noack, who recently left the institute.

Finally, I want to thank the members of my thesis committee-Burkhard Geil, Alec Wodtke, Konrad Koszinowski and Thomas Zeuch-for taking the time to evaluate my work. 



\section{Contents}

$\begin{array}{ll}\text { Introduction } & \mathbf{1}\end{array}$

1 Experimental and Theoretical Methods 3

1.1 Vibrational Anharmonicity . . . . . . . . . . . . . 3

1.2 Experimental Methods . . . . . . . . . . . . . . 8

1.2.1 Supersonic Expansions . . . . . . . . . . . . 8

1.2.2 The "Filet" Jet . . . . . . . . . . . . . . . . . . . . 11

1.2.3 MAX-lab Matrix Isolation Setup . . . . . . . . . . . . . 16

1.3 Theoretical Methods . . . . . . . . . . . . . . . . . 16

1.3.1 Approximations in Electronic Structure Methods . . . . 17

1.3.2 Anharmonic Calculations . . . . . . . . . . . 21

1.3.3 Software Packages and Basis Sets . . . . . . . . . 23

2 Vibrational Dynamics in Methanol Monomers and Dimers 27

2.1 Torsion-Stretching Dynamics in Methanol Monomers . . . . . . 29

2.1.1 Torsional Localization Models . . . . . . . . . . . . . . 32

2.2 Jet- and Matrix-FTIR Spectroscopy . . . . . . . . . . . . . . 38

2.2.1 Filet-Jet Spectra . . . . . . . . . . . . . . . . 39

2.2.2 Matrix-FTIR Spectra . . . . . . . . . . . . . 41

2.3 Quantum Chemical Calculations ... . . . . . . . . 45

2.3.1 Anharmonic Calculations . . . . . . . . . 52

2.4 OH Dimerization Red Shift . . . . . . . . . . . . . 58

2.5 Summary ........................ 59

3 Extension to Weak and Strong Hydrogen Bonds 63

3.1 Methanol-Ethene . . . . . . . . . . . . . . . . . . 64

3.1 .1 Jet-FTIR Spectra . . . . . . . . . . . . 65

3.1.2 Quantum Chemical Calculations . . . . . . . . . 67

3.1.3 OH Dimerization Red Shift . . . . . . . . . . . 71

3.2 Concurrent Docking Motifs in Methanol-Anisole Dimers . . . . 73

3.2 .1 Spectroscopic Evidence . . . . . . . . . . . . 73 
3.2.2 Harmonic and Anharmonic Predictions . . . . . . . . 77

3.3 Related $\mathrm{OH}$... O Hydrogen Bonds in Aliphatic Alcohols . . . . 79

3.3 .1 Ethanol .................... 81

3.3 .2 tert-Butyl Alcohol . . . . . . . . . . . . . 82

3.4 Summary ....................... 83

$\begin{array}{lr}\text { Summary } & 85\end{array}$

$\begin{array}{lll}\text { Appendix A } & \text { Experimental Parameters } & 87\end{array}$

$\begin{array}{lll}\text { Appendix B Numeric Matlab Routines } & 91\end{array}$

B.1 Outline and Source Code Excerpts . . . . . . . . . . . . . 91

B.2 Parameters . . . . . . . . . . . . . . . . . . . 94

B.2.1 Methanol Monomer Torsion . . . . . . . . . . . . 94

B.2.2 OH Stretching Vibrations . . . . . . . . . . . 95

Appendix C MoLPro Inputs and LMOMO Calculations $\quad 97$

C.1 Explicit Correlation . . . . . . . . . . . . . . . . 97

C.2 Spin-Component Scaling . . . . . . . . . . . . . . . 98

C.3 LMOMO Calculations . . . . . . . . . . . . . . . . . . . 99

$\begin{array}{lll}\text { Appendix D VPT2 Stability Tests } & 101\end{array}$

$\begin{array}{ll}\text { Bibliography } & 105\end{array}$ 


\section{Introduction}

Supersonic expansions are well-established today as a tool to isolate monomers and small clusters of molecules and study their intra- and intermolecular interactions. ${ }^{1-3}$ In combination with vibrational spectroscopy, it is possible to detect characteristic changes in the potential energy hypersurfaces that are induced by the formation of non-covalent contacts. For the ubiquitous $\mathrm{X}-\mathrm{H} \cdots \mathrm{Y}$ hydrogen bond (where $\mathrm{X}$ and $\mathrm{Y}$ are $\mathrm{N}, \mathrm{O}, \ldots$ atoms), the distinct spectroscopic "red shift" induced in the donor $\mathrm{X}-\mathrm{H}$ hydride stretching frequency constitutes the most sensitive and widely-used probe for this sort of interaction. The importance of this effect is mirrored by the fact that it has been included in the most recent 2011 IUPAC definition of the hydrogen bond itself,, 4,5 and empirical relationships have been proposed to correlate the observable red shift and intensity modulation with the strength of the hydrogen bond. ${ }^{6-8}$ However, the underlying structural motifs of the molecular aggregates are typically encoded rather indirectly in their vibrational spectra, and one usually has to compare the observations to quantum chemical calculations in the hope of matching a specific spectroscopic response among a set of proposed conformers. However, the chosen vibrational signals may be too insensitive to discriminate between structural variations, and more sophisticated experimental methods may be called for in order to establish conformational selectivity.

One fundamental problem that arises on the theoretical side is the accuracy of the available quantum chemical models, which generally correlates steeply with the computational cost. This is further aggravated for accurate vibrational treatments by the need to sample large portions of the nuclear potential energy hypersurface, which in turn necessitates a large number of sufficiently high-level electronic structure calculations. ${ }^{9}$ As a compromise, one can either cut down on the accuracy of the vibrational part, the electronic part, or both. Despite a growing number of facilitating approximations being introduced to quantum chemical methods, even medium-sized systems are to this day most often treated by falling back to the harmonic vibrational model and cheaper semi-empirical electronic structure methods. Even for 
small systems, highly accurate anharmonic calculations are far from being routine. Of course, diminishing the accuracy of the calculations necessarily opens a gap to the experiment, and the predictions may soon fall short of the quantitative or even qualitative demands for spectroscopic interpretations and assignments.

Given the often intertwined constraints imposed by computational demand and spectroscopic complexity, experimental benchmarks of anharmonic predictions must rely on the study of small and relatively simple systems. One such candidate is the methanol molecule as the smallest saturated alcohol with a single spectrally isolated $\mathrm{OH}$ stretching mode. Its monomer and homodimer have been investigated experimentally to great detail, ${ }^{10-13}$ but gas-phase information on anharmonicity in the latter has only recently been obtained. ${ }^{14}$ The prototype character of the methanol molecule brings it into focus also for the present work, which will revisit it for high-level harmonic calculations ${ }^{15}$ and further anharmonic treatments in a vibrational subspace. ${ }^{16}$ In addition to the gas-phase experiments, a number of matrix spectra sheds light on some important vibrational dynamics in this system. ${ }^{17}$ As a weaker sibling to this moderately strong interaction, the first-ever experimental investigation of the hydrogen bond between methanol and ethene will be presented and likewise characterized computationally. ${ }^{16}$

Using these two prototypical $\mathrm{OH} \cdots \mathrm{O}$ and $\mathrm{OH} \cdots \pi$ hydrogen bonds as bracketing cases, an investigation on the complex between methanol and anisole has allowed for a definite structural assignment of the observed bands. In addition, the analysis of harmonic and anharmonic effects is extended in the last section of this work to the dimers of ethanol and tert-butyl alcohol, and some general conclusions will be drawn from the assembled data. 


\section{Chapter 1}

\section{Experimental and Theoretical Methods}

In this section, a short overview of the theoretical background on vibrational anharmonicity shall be provided, followed by a description of the experimental and theoretical methods employed throughout this work. Specifically, the use of supersonic expansions for the study of intermolecular interactions is reviewed, and its specific application in the "filet" jet apparatus outlined. In addition, a short description of the matrix-FTIR setup used for some of the methanol dimer results is included. On the theoretical side, the concepts behind local and explicit correlation are introduced, together with a short treatment of anharmonic vibrational calculations.

\subsection{Vibrational Anharmonicity}

In the harmonic approximation, the vibrational modes of a polyatomic system are described by quadratic potential functions which are completely decoupled from one another. The resulting term energies of each normal mode thus depend linearly on a single vibrational quantum number. The simplest picture beyond this approximation includes linear two-mode or quadratic one-mode contributions; based on second-order perturbational theory in up to quartic vibrational potentials, ${ }^{18,19}$ the term energies in a system of $N$ normal modes take the form

$$
G\left(\mathrm{v}_{1}, \mathrm{v}_{2}, \ldots, \mathrm{v}_{N}\right)=\sum_{i}^{N} \omega_{i}\left(\mathrm{v}_{i}+\frac{1}{2}\right)+\sum_{i \leq j}^{N, N} x_{i, j}\left(\mathrm{v}_{i}+\frac{1}{2}\right)\left(\mathrm{v}_{j}+\frac{1}{2}\right),
$$

where $\mathrm{v}_{i}$ and $\omega_{i}$ are the vibrational quantum number and harmonic wavenumber of mode $i$, respectively. For $i=j$, the "diagonal" anharmonic- 
ity constants $x_{i, i}$ describe the effect of a non-quadraticity in the vibrational potential of mode $i$, while the "off-diagonal" anharmonicity constants $x_{i, j}$ introduce pairwise potential couplings between different normal modes $i$ and $j$. ${ }^{*}$ For any given mode $i$, the wavenumber of the $n$-th transition originating in the ground state is thus

$$
(n \tilde{v})_{i}=n \omega_{i}+\left(n^{2}+n\right) x_{i, i}+\frac{n}{2} \sum_{j \neq i} x_{i, j}
$$

and more specifically for the fundamental and first overtone transitions,

$$
\begin{aligned}
\tilde{v}_{i} & =\omega_{i}+2 x_{i, i}+\frac{1}{2} \sum_{j \neq i} x_{i, j}, \\
(2 \tilde{v})_{i} & =2 \omega_{i}+6 x_{i, i}+\sum_{j \neq i} x_{i, j} .
\end{aligned}
$$

The diagonal anharmonicity content $x_{i, i}$ of the respective mode can be obtained from these two transitions by

$$
x_{i, i}=\frac{1}{2}(2 \tilde{v})_{i}-\tilde{v}_{i}
$$

For two-mode combination and hot bands with $\left(\mathrm{v}_{i}, \mathrm{v}_{j}\right)=(1,1) \leftarrow(0,0)$ and $(1,1) \leftarrow(0,1)$, respectively, one obtains

$$
\begin{aligned}
& \tilde{v}^{(1,1) \leftarrow(0,0)}=\tilde{v}_{i}+\tilde{v}_{j}+x_{i, j}, \\
& \tilde{v}^{(1,1) \leftarrow(0,1)}=\tilde{v}_{i}+x_{i, j} .
\end{aligned}
$$

Upon formation of an $\mathrm{X}-\mathrm{H} \cdots \mathrm{Y}$ hydrogen bond, several effects on the vibrational dynamics in the donor bond are noticeable. Polarization effects weaken the $\mathrm{X}-\mathrm{H}$ bond, decreasing its harmonic wavenumber and increasing the diagonal anharmonicity in magnitude. The latter can be probed by observing the first overtone of the stretching vibration in the cluster according to Equation (1.5).

The infrared absorption of a vibrational transition between two states $\mathrm{v}_{i}^{\prime} \neq \mathrm{v}_{i}^{\prime \prime}$ is governed by the associated transition dipole moment. In a quantum mechanical picture, this can be calculated as the expectation value of the dipole moment operator, $\hat{\mu}$. Dunham ${ }^{20}$ first proposed to expand the dipole moment $\mu$ in a Taylor expansion around the equilibrium geometry, which allows to express the expectation value in a shorthand notation as

$$
\langle\hat{\mu}\rangle_{i}^{\mathrm{v}^{\prime} \leftarrow \mathrm{v}^{\prime \prime}}=\left\langle\mathrm{v}_{i}^{\prime}|\hat{\mu}| \mathrm{v}_{i}^{\prime \prime}\right\rangle=\partial_{i} \mu\left\langle\mathrm{v}_{i}^{\prime}\left|Q_{i}\right| \mathrm{v}_{i}^{\prime \prime}\right\rangle+\frac{1}{2} \partial_{i i} \mu\left\langle\mathrm{v}_{i}^{\prime}\left|Q_{i}^{2}\right| \mathrm{v}_{i}^{\prime \prime}\right\rangle+\ldots
$$

\footnotetext{
*The denominations as "diagonal" and "off-diagonal" terms stem from the positions of the elements when arranged in a matrix.
} 
where $Q_{i}$ is the vibrational coordinate associated with the considered normal mode, $\mathrm{v}_{i}$ represents the vibrational wavefunction $\psi_{\mathrm{v}}\left(Q_{i}\right)$, and $\partial_{i}$ denotes the partial derivative $\partial / \partial Q_{i}$ (with analogous second derivatives $\partial_{i i}$ ). Without loss of generality, orthonormal wavefunctions can be assumed so that the zeroth-order term of the expansion-containing any permanent dipole moment-vanishes.

Following the Beer-Lambert law, the observable integrated absorbance of a vibrational band centered at $\tilde{v}_{i}$ is given by ${ }^{21}$

$$
\int A \mathrm{~d} \tilde{v}=\int \kappa(\tilde{v}) \mathrm{d} \tilde{v} \cdot c d
$$

Here, $c$ is the sample concentration, $d$ the optic absorption path length, and $\kappa$ the molar extinction coefficient which depends on the transition dipole moment as ${ }^{21}$

$$
\int \kappa(\tilde{v}) \mathrm{d} \tilde{v}=\frac{8 \pi^{3}}{3 h c\left(4 \pi \varepsilon_{0}\right)} N_{\mathrm{A}} \tilde{v}_{i}\left|\langle\hat{\mu}\rangle_{i}^{\mathrm{v}^{\prime} \leftarrow \mathrm{v}^{\prime \prime}}\right|^{2} .
$$

In more accurate treatments, the rotational structure of the band in question must be included by forming a sum over all contributing rovibrational transition dipole moments and multiplying the expression by appropriate Boltzmann factors. Likewise, contributions from hot bands that are spectrally close to the fundamental need to be included in the absorption intensity. However, the errors from neglecting both contributions can be assumed to be small, especially if a low population of excited vibrational states disfavors hot band transitions. ${ }^{22-24}$

In general, the polarizing influence of a hydrogen bond aids in the detection of an $\mathrm{X}-\mathrm{H}$ stretching fundamental band since the leading first dipole derivative, $\partial_{i} \mu$, drastically increases. Conversely, the intensity of the first overtone band, which is already inherently weaker than the fundamental itself, is often found to decrease even further due to the hydrogen bond. ${ }^{14,25}$ Intuitively, one might ascribe this to a diminishing in electric anharmonicity, i.e. the second derivative $\partial_{i i} \mu$, since this term is also responsible for lending the overtone transition its intensity in the first place. However, Di Paolo et $a l .{ }^{26}$ have investigated this trend based on second-order perturbational treatments in quartic potentials, using analytic transition dipole moment formulas as derived by Herman and Shuler. ${ }^{27}$ Their findings suggest that the observable low overtone intensity is not primarily due to a decreasing second dipole derivative; in fact, its contribution has to be of sizable magnitude to explain the overall effect. Rather, the resulting intensity expressions involve some compensation among the first and second derivatives. The increase in the first derivative-responsible for boosting the fundamental intensity-is in itself partly responsible for the overtone attenuation, and 
the second derivative must be of equal sign and non-vanishing to explain the observable effects (whereas it is typically of opposite sign in the absence of a hydrogen bond). This also implies opposite signs for the corresponding $\left\langle 2\left|Q_{i}\right| 0\right\rangle$ and $\left\langle 2\left|Q_{i}^{2}\right| 0\right\rangle$ integrals in Equation (1.8). Calculations on the corresponding terms in hydrogen-bonded complexes have been carried out by Kjaergaard et al. ${ }^{28}$ for various water aggregates and by Scharge et al. ${ }^{29}$ for trifluoroethanol, confirming this trend at least for strong hydrogen bonds. Within this work, the intensity effects will be exemplified for the methanol dimer throughout Chapter 2, and for the methanol-ethene aggregate in Section 3.1.

While the donor $\mathrm{X}-\mathrm{H}$ group exhibits a distinct weakening of its stretching force constant from the influence of the acceptor site, the opposite is true for its torsional motion perpendicular to the hydrogen bond. Excitation of this "librational" motion tends to break the hydrogen bond, diminishing the acceptor's grip on the donor bond and thus blue-shifting its stretching wavenumber towards the non-bonded case. ${ }^{\dagger}$ An experimental determination of this important coupling constant requires to observe either combination or hot bands, in addition to one or both fundamentals, as indicated by Equations (1.6) and (1.7). Unfortunately, the free torsion and confined libration bands appear at low wavenumbers in the far-infrared (FIR) region which is somewhat difficult to access experimentally. Observing the stretching-libration hot band according to Equation (1.7) would be favorable in this regard, since only the stretching fundamental is required to obtain the cross-coupling constant.

The transition dipole moments that drive the IR intensity of combination and hot bands can be expressed in a two-dimensional extension of Equation (1.8) when using linearly independent normal modes. With labels "s" and "l" for the stretching and libration bands, respectively, and bra-ket terms

\footnotetext{
†One may argue that the term "libration" describes the rotation of a quasi-rigid body against a restoring potential, and that for an organic alcohol, the vibration in question should rather be denoted a hindered torsion. In the present work however, the term "libration" will be retained for two reasons. First, these vibrations are typically found at energies around a few $100 \mathrm{~cm}^{-1}$, comparable to the "true" librational motion in e.g. water; conversely, the rigid counter-rotation lies much lower in energy and depends strongly on the molecular mass. Further, it allows an easy distinction from the much less-hindered $\mathrm{OH}$ torsion in the acceptor molecule.
} 
sorted by stretching terms first, then libration terms, one obtains

$$
\begin{array}{r}
\langle\hat{\mu}\rangle_{\mathrm{sl}}^{(1,1) \leftarrow(0,0)}=\partial_{\mathrm{s}} \mu\left\langle 1\left|Q_{\mathrm{s}}\right| 0\right\rangle\langle 1 \mid 0\rangle+\langle 1 \mid 0\rangle \partial_{1} \mu\left\langle 1\left|Q_{1}\right| 0\right\rangle+\frac{1}{2} \partial_{\mathrm{ss}} \mu\left\langle 1\left|Q_{\mathrm{s}}^{2}\right| 0\right\rangle\langle 1 \mid 0\rangle \\
+\langle 1 \mid 0\rangle \frac{1}{2} \partial_{\mathrm{ll}} \mu\left\langle 1\left|Q_{1}^{2}\right| 0\right\rangle+\partial_{\mathrm{sl}} \mu\left\langle 1\left|Q_{\mathrm{s}}\right| 0\right\rangle\left\langle 1\left|Q_{1}\right| 0\right\rangle, \\
\langle\hat{\mu}\rangle_{\mathrm{sl}}^{(1,1) \leftarrow(0,1)}=\partial_{\mathrm{s}} \mu\left\langle 1\left|Q_{\mathrm{s}}\right| 0\right\rangle\langle 1 \mid 1\rangle+\langle 1 \mid 0\rangle \partial_{1} \mu\left\langle 1\left|Q_{1}\right| 1\right\rangle+\frac{1}{2} \partial_{\mathrm{ss}} \mu\left\langle 1\left|Q_{\mathrm{s}}^{2}\right| 0\right\rangle\langle 1 \mid 1\rangle \\
+\langle 1 \mid 0\rangle \frac{1}{2} \partial_{11} \mu\left\langle 1\left|Q_{1}^{2}\right| 1\right\rangle+\partial_{\mathrm{sl}} \mu\left\langle 1\left|Q_{\mathrm{s}}\right| 0\right\rangle\left\langle 1\left|Q_{1}\right| 1\right\rangle .
\end{array}
$$

Orthonormalized wavefunctions can again be assumed without loss of generality so that all $\left\langle\mathrm{v}^{\prime} \mid \mathrm{v}^{\prime \prime}\right\rangle$ terms can be replaced by a Kronecker delta $\delta_{\mathrm{v}^{\prime} \mathrm{v}^{\prime \prime}}$, yielding

$$
\begin{aligned}
& \langle\hat{\mu}\rangle_{\mathrm{sl}}^{(1,1) \leftarrow(0,0)}=\partial_{\mathrm{s} l} \mu\left\langle 1\left|Q_{\mathrm{s}}\right| 0\right\rangle\left\langle 1\left|Q_{1}\right| 0\right\rangle, \\
& \langle\hat{\mu}\rangle_{\mathrm{sl}}^{(1,1) \leftarrow(0,1)}=\partial_{\mathrm{s}} \mu\left\langle 1\left|Q_{\mathrm{s}}\right| 0\right\rangle+\frac{1}{2} \partial_{\mathrm{ss}} \mu\left\langle 1\left|Q_{\mathrm{s}}^{2}\right| 0\right\rangle+\partial_{\mathrm{sl}} \mu\left\langle 1\left|Q_{\mathrm{s}}\right| 0\right\rangle\left\langle 1\left|Q_{1}\right| 1\right\rangle .
\end{aligned}
$$

Assuming a more or less symmetric vibrational potential for the torsional motion, the $\left\langle 1\left|Q_{1}\right| 1\right\rangle$ terms in Equation (1.12) will approximately vanish; the remainder of the expression then just reflects the strong stretching fundamental transition, $\partial_{\mathrm{s}} \mu\left\langle 1\left|Q_{\mathrm{s}}\right| 0\right\rangle+\frac{1}{2} \partial_{\mathrm{ss}} \mu\left\langle 1\left|Q_{\mathrm{s}}^{2}\right| 0\right\rangle$. Together with the similar wavenumbers for the stretching fundamental and the stretching-libration hot band (Equation (1.7)), their molar extinction coefficients as per Equation (1.10) should be roughly equal. However, the intensity of the librationally hot band depends directly on the population of the first excited state, and for a hypothetical wavenumber around $600 \mathrm{~cm}^{-1}$ at vibrational temperatures of 100 to $200 \mathrm{~K}$, the population relative to the ground state would be on the order of only $10^{-2}$ to $10^{-3}$. Thus, although the extinction coefficients of the hot band would favor it over the combination band for the determination of the anharmonicity constant $x_{\mathrm{s}, \mathrm{l}}$, its detectability may be greatly hampered under vibrationally cold conditions such as in supersonic jets or cryogenic matrices unless a significant amount of the sample can be prepared in the librationally excited state, e.g. through optical pumping.

Finally, it should be noted that the above expressions for the $\hat{\mu}$ expectation values are useful only if a second-order Taylor expansion provides a viable approximation to the dipole curves in the regions of space sampled by the participating wavefunctions. If higher orders are necessary to capture the essence of the dipole (hyper-)surface, numerical treatments across larger ranges of displacements may be required, and the highlighted intensity effects may be either attenuated or exacerbated. 


\subsection{Experimental Methods}

In 1951, Kantrowitz and Grey ${ }^{30}$ proposed the creation of molecular flow fields by means of supersonic expansions, where a gas flow ("jet") is driven along a large pressure gradient through a small nozzle. Such beam sources promised advantages over common effusive techniques in terms of higher flow intensities and thermal cooling of the beam while allowing much narrower velocity distributions. Indeed, these favorable properties soon led to supersonic expansions being accepted as a viable tool for the study of molecules and molecular clusters in cold and sparse environments. Below, a general description of the properties of supersonic beam sources is given, based on the accounts by Morse ${ }^{31}$ and Herman et al. $;^{3}$ yet another comprehensive review is provided by Arnó and Bevan. ${ }^{32}$ As a concise entry point to the topic, a brief yet informative paper by Smalley in Reference 2 can be recommended. Further studies by Hagena ${ }^{33,34}$ detail on the condensation behavior in supersonic beams.

\subsubsection{Supersonic Expansions}

In an effusive beam, atoms or molecules from a gas reservoir pass through a small opening in their confinement solely based on random thermal motion. This limiting case comes into effect at low sample pressures when the mean free path of the particles in the reservoir is much larger than the confining dimension of the opening. Conversely, increasing the stagnation pressure in the reservoir lowers the mean free path of the gas particles until it reaches a regime where they experience a large number of collisions while passing through the outlet. Their overall thermal energy is then partly redistributed into the directed motion through the opening, leading to an internal cooling of the gas sample. Ongoing collisions in the expanding beam further equalize the relative particle velocities, corresponding to a low translational temperature in terms of a Maxwell-Boltzmann distribution. Consequently, the speed of sound inside the particle ensemble effectively decreases until it is surpassed by its absolute velocity: the gas flow becomes supersonic.

Possibly the most common application of supersonic beams today is the stabilization of small molecular or atomic aggregates for spectroscopic investigation. One prominent example for a species first isolated in a jet expansion is the elusive helium dimer. ${ }^{35}$ The technique has a decisive advantage over thermostatted environments which are limited in their applicability by progressive aggregation of the analytes both to condensation nuclei and the sample cell walls. Typically, aggregation experiments in supersonic expansions are carried out in "seeded" beams in which a small amount of the 
analyte is diluted in a carrier gas-most often a rare gas-which serves as a spectroscopically silent collisional-cooling agent. The cooling of the analyte molecules in the heat bath of the carrier gas is more efficient than in pure beams, and rotational temperatures drop low enough so that the spectroscopic signatures become vastly simplified. The concentration and size distribution of the clusters can be controlled by the dimensions of the outlet nozzle, seeding fraction of the analyte, composition of the carrier gas, stagnation pressure in the reservoir, and temperature of the gas sample. Further, the geometry of the nozzle can be designed so as to restrict the gas flow after the expansion, increasing the number of many-body collisions and promoting cluster formation. A similar effect can be achieved by using an elongated slit instead of a pinhole nozzle, limiting the outwards gas flow from the beam and leading to a slower density fall-off along the expansion. The longer absorption pathway permitted by a slit-nozzle expansion zone is further advantageous for the application of spectroscopic techniques. However, the inherently low particle densities in supersonic beams remain a fundamental and lasting problem in their application, prompting for highintensity light sources and sensitive detection strategies.

Due to the non-uniformity of the collisional energy redistribution, supersonic expansions represent non-equilibrium environments in which the translational, rotational and vibrational temperatures of the ensemble increase as $T_{\text {trans }} \leq T_{\text {rot }}<T_{\text {vib. }}$. For $T_{\text {trans }}$, values as low as a few milli-Kelvin have been reported, ${ }^{3}$ and typical values can be assumed to be in the low Kelvin to sub-Kelvin regime. If the number of collisions in the expansion is not a limiting factor, rotational cooling may be as efficient as its translational counterpart, but small molecules may retain rotational temperatures of up to a few tens of Kelvins in supersonic expansions. ${ }^{36}$ For the vibrational degrees of freedom, estimates become more difficult due to their substantial energy content and incomplete inter-mode equilibration, and the vibrational temperature $T_{\text {vib }}$ becomes ill-defined. This non-uniformity comes into play for conformational interconversion processes which are initialized by appropriate large-amplitude backbone molecular motions. For the facile and tunneling-assisted gauche-trans torsion coordinate in ethanol, conformational temperatures down to $c a .50 \mathrm{~K}$ were found in a previous Raman-jet study. ${ }^{37}$ However, an analogous characterization of trifluoropropanol ${ }^{38}$ suggested that relevant interconversion motions may easily become kinetically "frozen" at least for high-barrier, tunneling-deficient motions, and similar disequilibration was found for the dispersion-driven folding and unfolding of long alkane chains. ${ }^{39}$ The observable, jet-cooled conformer distributions are hence governed by complicated thermodynamic and kinetic characteristics of both the employed setup and the gas sample. 
Further downstream of the nozzle, the initially large number of collisions drops due to the spreading of the gas beam and an equalization of the particle velocities. A "zone of silence" is established where the energy redistribution in the expansion largely comes to a halt and the Mach number of the gas flow reaches its maximum. Finally, the "over-expanded" low-density beam is recompressed by collisions with residual gases in the apparatus, forming a terminal shock wave in which its temperature and density discontinuously rise to background levels. Similar lateral shock waves blanket the sides of the expansion, encasing the cold supersonic flow in regions of warmer boundaries. In order to avoid interference from these shock waves, the cold core of the expansion can be isolated by placing appropriate skimmers in the beam and passing the selected gas into a secondary vacuum chamber where further manipulations and detection can be carried out.

Most commonly, intermolecular interactions in supersonic jets are studied by means of vibrational spectroscopy, probing subtle changes of the constituent's potential energy surfaces from the monomeric to the aggregated situation. Direct laser absorption techniques have been implemented, ${ }^{40}$ although many newer experimental approaches employ multi-resonance laser excitations and mass-detection schemes, adding the advantage of size- or even conformer selectivity in the experiments. ${ }^{41}$ However, the number of vibronic states that are involved in the probing schemes bereaves such techniques of direct intensity information and may further obfuscate the desired vibrational signatures. One such case is apparent in a cyclohexanol study by Léon et al. ${ }^{42}$ who observed broad hydrogen-bonded $\mathrm{OH}$ stretching bands in IR/UV and IR/IR multi-resonance experiments, as opposed to the typical narrow patterns that were found through direct IR absorption.

Jet experiments can be designed for either continuous or pulsed operation. ${ }^{43}$ Since the position of the terminal recompression shock waves in a supersonic expansion is governed by the square-root ratio of the stagnation pressure in the gas reservoir and the residual background pressure in the jet chamber, pulsed jets may provide more well-defined expansions and more pronounced cooling behavior than continuously operated nozzles if the backing vacuum system is adequately sized and pumped between pulses. Buffering the pressure increase in the system thus permits large gas throughputs during each pulse and consequently high optical densities in the probing zone. However, the maximum attainable pulse duration imparts some limitations for interferometric sampling techniques in terms of spectroscopic resolution and scanning speed if the full interferogram is to be recorded during a single gas pulse. These limitations can be circumvented by step scanning, i.e. sectionally recording the interferogram during subsequent gas pulses, at the obvious cost of an increased time demand for a full scan. ${ }^{44}$ 
Finally, two interesting features of seeded supersonic beams shall not go unmentioned. First, particles with higher velocity components perpendicular to the expansion centerline naturally remove themselves from the core of the gas stream. Thereby, the "perpendicular" translational temperature in the beam center decreases as the expansion progresses. Since the width of the Maxwell-Boltzmann distribution (and thus the fraction of faster particles) decreases with increasing mass, the heavier component in a seeded beam tends to enrich at the core of the expansion. ${ }^{45}$ Second, a large mass disparity in a seeded expansion can cause the acceleration of the heavier component to lag behind the lighter one. This "velocity slip" effect may be pronounced enough to allow supersonic beam sources to be used for isotope separation by means of velocity selection. ${ }^{46,47}$

\subsubsection{The "Filet" Jet}

\section{Overview}

The supersonic beam apparatus used throughout this work, nicknamed the "filet" jet, was constructed in the context of the $\mathrm{PhD}$ thesis of Nicole Borho ${ }^{48}$ in 2003. Over the years, the apparatus has seen the exchange of the connected FTIR spectrometer and an extension of its realistic spectral range to $200-8000 \mathrm{~cm}^{-1} \cdot 14,29,49,50$ A recent account of its features and operation is given in Reference 43, together with a demonstration of its capabilities for the measurement of $\mathrm{O}-\mathrm{H}$ stretching fundamental and overtone bands. The following description will thus be restricted to a brief overview of the setup, noting only important key points and changes to previously established routines.

The unmatched eponymous feature of the setup is the "fine, but lengthy" slit nozzle of $600 \times 0.2 \mathrm{~mm}^{2}$ dimensions with a throughput of up to $3 \mathrm{~mol} \mathrm{~s}^{-1}$, backed by a buffer volume of up to $23 \mathrm{~m}^{3}$ and a pumping system of up to $2500 \mathrm{~m}^{3} \mathrm{~h}^{-1}$. Six solenoid valves pass the sample gas mixture from a $67 \mathrm{~L}$ reservoir into a pre-expansion chamber which serves to ensure a spatially homogeneous feeding of the nozzle. The large absorption pathway through the elongated expansion zone allows convenient sampling at a variable downstream distance by the mildly focused beam of an unmodified, evacuated Bruker IFS 66v/S FTIR spectrometer and its built-in light sources. ${ }^{51}$ Measurements in the spectral $\mathrm{OH}$ and OD fundamental region of $c a$. 4000$2400 \mathrm{~cm}^{-1}$ are possible using a tungsten light source, $\mathrm{KBr}$ or $\mathrm{CaF}_{2}$ optics and beamsplitters, and an external $\mathrm{LN}_{2}$-cooled $3 \mathrm{~mm}^{2} \mathrm{InSb}$ detector; for the measurement of the respective stretching overtones between 7500 and $6400 \mathrm{~cm}^{-1}$, a $7 \mathrm{~mm}^{2}$ InGaAs detector is available. The mid- and far-infrared 


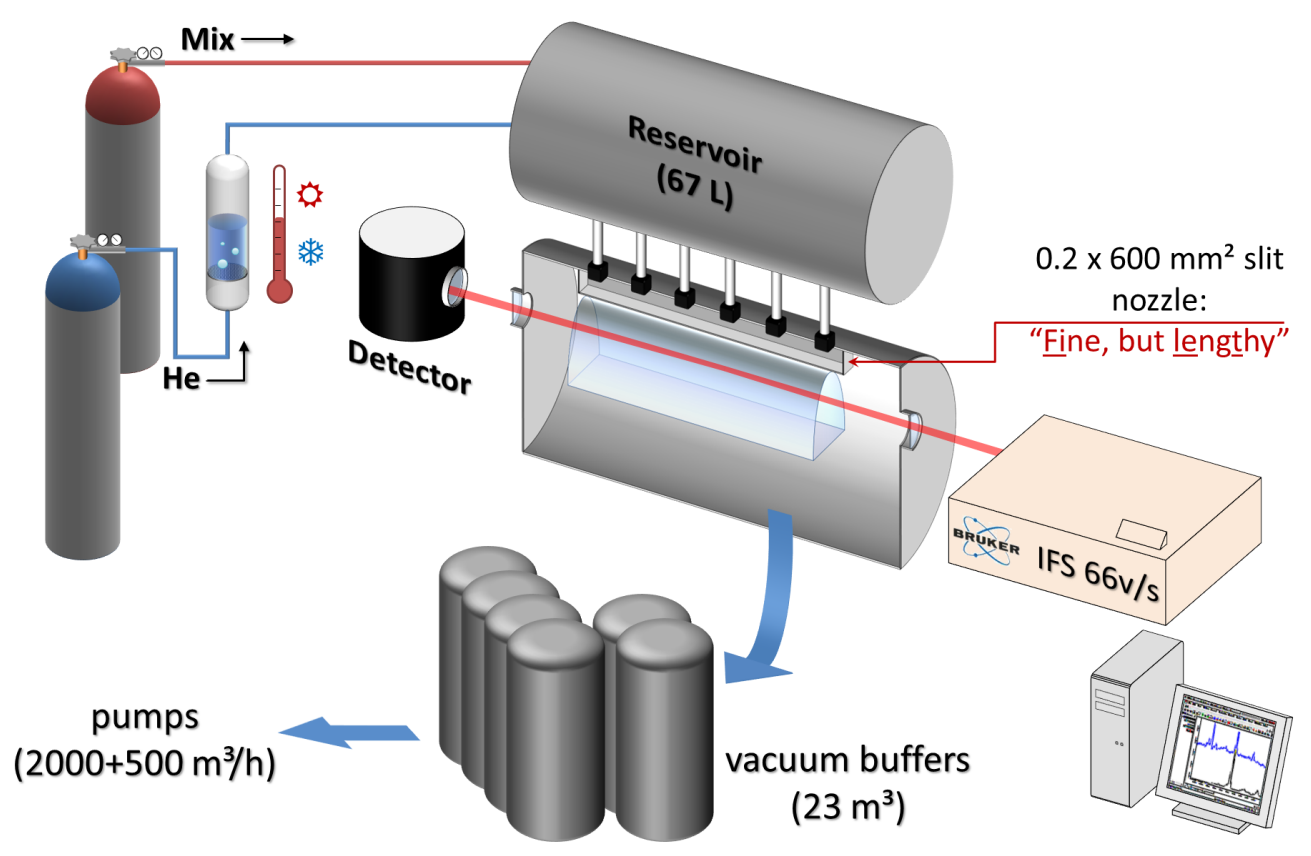

Figure 1.1: Schematic of the "filet" jet (not to scale).

(MIR and FIR) regions are accessible by MCT detectors or a liquid heliumcooled bolometer in conjunction with $\mathrm{KBr}$ or Mylar beamsplitters; these were, however, not put to use for the present work. In all cases, appropriate optical filters allow to reduce the bandwidth of the detector and increase the signal-to-noise ratio. The jet valves are synchronized to the rapid scans of the FTIR spectrometer, with typical pulse durations on the order of $150 \mathrm{~ms}$ followed by an evacuation period of 30 to $60 \mathrm{~s}$.

Sample preparation is carried out in thermostatted glass saturators by directing a stream of the carrier gas through the liquid or solid analyte. By varying the carrier gas pressure, saturator temperature, and opening/closing times of the feeding solenoid valves, the concentration of the sample can be controlled. For more concentration-sensitive measurements or gaseous analytes, a mixing line is available to prepare more well-defined sample mixtures in a $50 \mathrm{~L}$ gas cylinder which can be fed directly into the reservoir.

One advantage of the filet jet over its size- or even conformer-selective multi-resonance siblings is the reliance on direct infrared absorption, allowing band positions as well as intensity information to be extracted from the recorded spectra. Both quantities provide estimates for the strength of 
hydrogen-bonded stretching modes (see Section 1.1) and can serve as sensitive benchmarks for quantum chemical predictions. Observing the weak overtone bands of hydrogen-bonded stretching vibrations, however, proves somewhat elusive due to their inherent intensity penalty outlined above. Ultimately, this mandates the measurement times (or equivalently, the number of co-added jet scans) to be increased by about one order of magnitude as compared to the fundamental bands. Moreover, determining the intensity ratio between fundamental and overtone bands necessitates recording a number of additional panoramic spectra over their combined spectral regions, using the monomer bands as an internal standard for calibration of the different optical filters and detectors. The intensity ratio thus provides an additional, but somewhat remote means of assessing the interaction strength, and its determination is not a routine task even in the straightforward filet jet experiment. Within this work, the method has been put to direct use in ambiguous methanol-anisole experiments to validate the assignment of competing structural motifs, ${ }^{52}$ as will be presented in Section 3.2.

\section{Modifications}

Some minor changes to the setup were made during the course of this thesis. First, a Teflon coating was applied to the inside of the reservoir in order to prevent issues from corrosion of the stainless-steel tank. However, a certain amount of adsorption of the sample gas by the coating itself is detectable, and the reservoir must be thoroughly evacuated after measurements and before changing sample mixtures. Second, the standard $12 \mathrm{~V}, 50 \mathrm{~W}$ tungsten lamps employed previously were exchanged for higher-power $24 \mathrm{~V}, 150 \mathrm{~W}$ analogs in order to facilitate fundamental and overtone experiments. The resulting signal-to-noise capabilities of the two light sources can be judged from a standardized "NOTCH" ("Noise Test Challenge") noise level analysis. In this routine, the jet setup is run up to a normal measurement-ready state in the desired configuration. 1-minute background and sample scans are recorded through the empty jet chamber, and the resulting absorbance spectrum is sent to a custom-made FORTRAN program which calculates a quadratic fit to the spectral baseline and its root-mean-square error (RMSE) in a $50 \mathrm{~cm}^{-1}$ moving window. Its negative decadic logarithm is stored for each window center position as a figure of merit for the noise level in the according region, allowing direct comparison of measurement configurations and different spectrometers as well as long-term stability monitoring.

Figure 1.2 shows a set of NOTCH curves obtained using external InSb and InGaAs detectors in typical measurement configurations with $150 \mathrm{~W}$ and $50 \mathrm{~W}$ tungsten lamps. Two measurements were carried out for each, and 

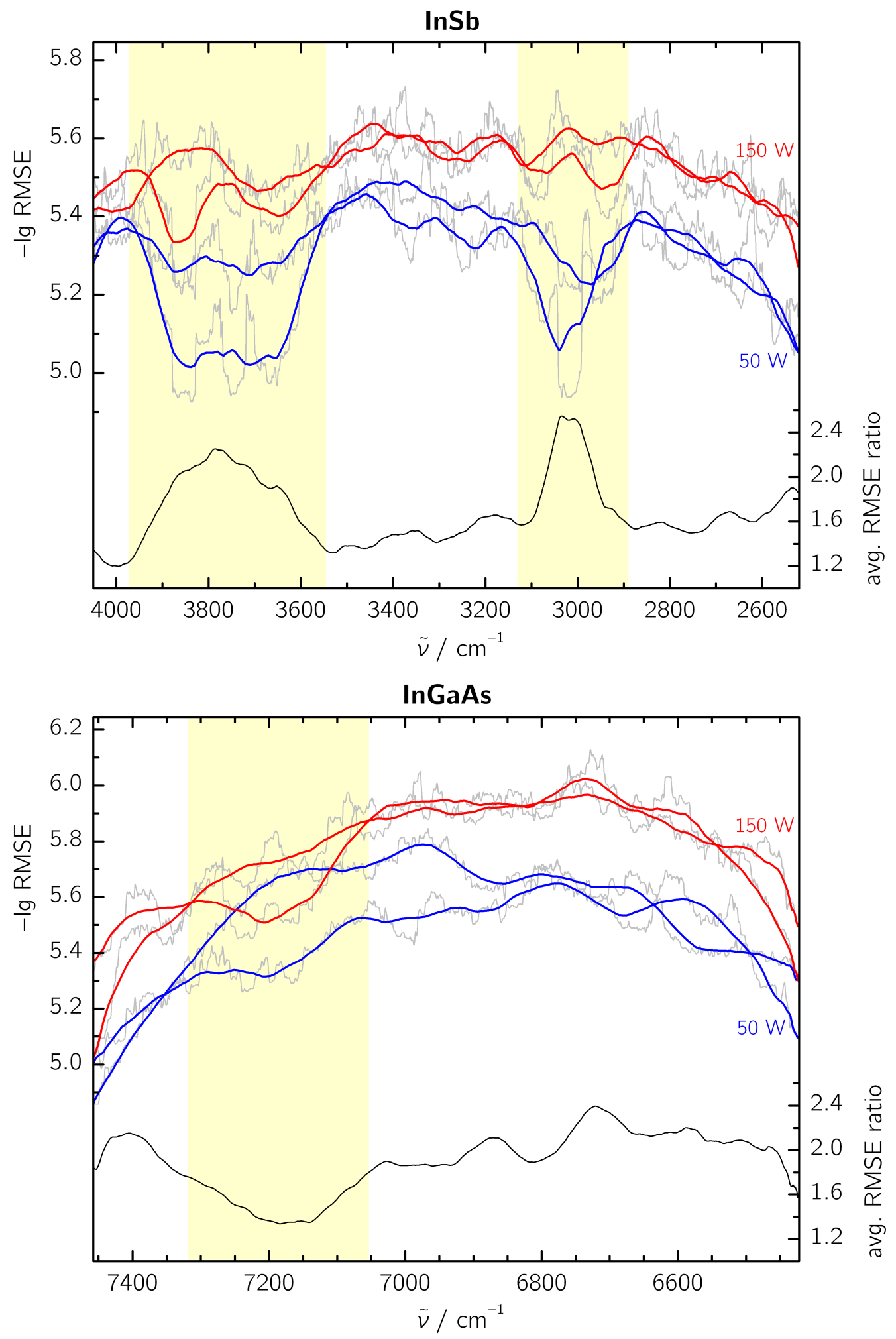
the original data (gray curves) were smoothed to a 200-point moving mean ( $\sim 100 \mathrm{~cm}^{-1}$, with red and blue curves for 150 and $50 \mathrm{~W}$ lamps, respectively). The yellow shading marks regions in which residual water and methane bands impair the analysis. The lower traces in each graph shows averaged $150 \mathrm{~W} / 50 \mathrm{~W}$ RMSE ratios and demonstrate the advantage of the stronger light source across both spectral regions by a factor of $\sim 1.5$ in the noise level. Additional details on these measurements are given in Appendix A.

The standard $80 \mathrm{kHz}$ velocity setting for the scanning interferometer mirror had previously been reduced to $60 \mathrm{kHz}$ in overtone measurements due to unfavorable noise impairments at the higher setting. For the overtone measurements conducted within this work, the $80 \mathrm{kHz}$ option was revisited with the $150 \mathrm{~W}$ tungsten source. Two independent error sources were isolated which produced excessive baseline noise in the near-infrared region through ill-defined compensation of water vapor bands between the background and sample scans. It can be assumed that the measures undertaken to eliminate these problems also take effect in the fundamental region, and they have thus been implemented permanently.

First, the scanning mirror is reset to an initial position by the controlling software at the beginning of each measurement cycle; its motion is then started, and a number of background scans is recorded. This stoppingstarting event apparently leads to mechanical vibrations of the mirror which spoil the first background scans, consequently causing artifacts in all absorbance spectra. To circumvent this problem, a "Wait 5000" command line was added to the top of the TRS routine that controls the synchronization of the spectrometer with the jet setup. This pre-scan delay leads to five seconds of "blind" mirror motion, thus allowing the disturbances to settle before commencing the data acquisition for the background scans.

Second, the front face of the nozzle base plate attached to the preexpansion chamber partially extends into the IR beam under typical experimental conditions. Although the respective part of the construction is blackened, a portion of this light still appears to be scattered either back into the interferometer or into the detector chamber. Upon releasing the jet

Figure 1.2 (opposite): NOTCH curves for $150 \mathrm{~W}$ (red) and $50 \mathrm{~W}$ (blue) tungsten lamp comparison using InSb (top) and InGaAs (bottom) detectors and appropriate optical filters. The lower traces in each graph show averaged $150 \mathrm{~W} / 50 \mathrm{~W}$ RMSE ratios. Yellow shading indicates regions where residual water and methane bands may impair the noise level analysis. 
gas pulse, the nozzle starts to vibrate, modulating this scattered light and leaving similar noise artifacts as described above. These artifacts vanish when removing the nozzle from the beam or reducing the vibrations by lowering the stagnation pressure. As a simple solution, it is sufficient to block the offending part of the IR beam from entering the jet chamber by partially covering the entrance window.

\subsubsection{MAX-lab Matrix Isolation Setup}

In addition to the jet-FTIR experiments conducted at the filet jet, a matrix isolation setup situated at the MAX-lab facility in Lund, Sweden, was employed in cooperation with the group of Wugt Larsen from the Technical University of Denmark in Copenhagen. Details about the setup can be found in References 53 and 54. In short, it features a gold-plated copper mirror cooled to $2.8 \mathrm{~K}$ by a helium cryostat. A wide spectral range is accessible using $\mathrm{KBr}$ and $\mathrm{CaF}_{2}$ beamsplitters together with InSb and MCT detectors. Further Mylar/bolometer options and different substrate window materials are available to sample the FIR region, but were not employed for the present work. The deposited matrix is sampled through a CsI window by a Bruker IFS 120 FTIR spectrometer at typical resolutions of 0.1 to $0.5 \mathrm{~cm}^{-1}$. Annealing is possible by resistively heating the matrix up to about $9 \mathrm{~K}$ in order to promote the mobility and conformational flexibility of the embedded guest molecules.

\subsection{Theoretical Methods}

One of the fundamental problems in theoretical chemistry is the often prohibitively steep scaling of their computational demand with respect to the attainable accuracy. Due to the many-body nature of all but the simplest quantum systems, their treatment must rely on iterative and approximative methods. This typically entails first building a Hartree-Fock reference wavefunction and subsequently recovering the electron correlation energy in dedicated calculations. The typical basis set expansions for the one-electron orbitals prompt for the handling of a large amount of data, and practical issues of memory allocation and disk swapping may become the bottlenecks of a calculation. Different approximations exist to widen these bottlenecks either by reducing the data quantity per se, or by facilitating its handling in a practical sense. A brief overview of three relevant techniques-Density Fitting, local and explicit correlation treatments-will be given in the first subsection of this chapter. Still, most standard implementations of $a b$ ini- 
tio methods are already prohibitively expensive for medium-sized molecular systems, let alone applications at the macromolecular scale.

Tackling vibrational dynamics introduces yet another range of problems. On grounds of the Born-Oppenheimer approximation, the overall molecular wavefunction can be separated into dedicated electronic and nuclear problems, and building a potential energy hypersurface entails solving the electronic Schrödinger equation (or some approximation thereof) at a large number of nuclear configurations. Even within only the harmonic approximation, this already imposes a quadratically-scaling computational demand for calculation of the Hessian matrix of a molecular system. Analytic implementations of energy gradient calculations help to alleviate this demand, but are not universally available across quantum chemical methods, as will be further discussed below. Worse, the harmonic approximation is clearly insufficient for direct quantitative and often even qualitative comparison to experimental results. The remaining gap must be closed by introducing anharmonic effects into the calculations, and although such treatments can often be truncated to some lower-order corrections, the resource and time demand becomes even more drastically dependent on the system size. Reduceddimensionality variational or, alternatively, perturbational approaches may relax these constraints somewhat. Still, thorough anharmonic treatments are far from being routinely applicable beyond rather small systems or simple electronic structure methods. The primary leverage in making larger molecular systems accessible to accurate vibrational calculations lies in reducing the computational demand of the underlying electronic structure problems, and some strategies to this end will be discussed below.

\subsubsection{Approximations in Electronic Structure Methods}

\section{Density Fitting}

During the evaluation of electron-electron interactions in electronic structure calculations, large amounts of four-index integrals over the molecular orbitals need to be calculated and transformed. One technique to reduce the memory demand of these steps is Density Fitting (DF) ${ }^{55-57}$ in which orbital products $\phi_{i} \phi_{j}$ are interpreted as an electron density and expanded in an auxiliary basis set. In this way, the integrals can be reduced to twoand three-index forms, which simplifies their handling. ${ }^{57,58}$ Usual implementations of the method rely on prefabricated basis sets for application in different methods, and a number of optimized sets are available for use in conjunction with popular atomic orbital (AO) basis sets.

Density Fitting is often synonymously called "Resolution of the Identity" 
(RI) due to its similarity to the mathematical procedure of the same name. However, RI expansions are of further importance in explicitly correlated methods (see below). To distinguish between both concepts, the present work follows the established nomenclature ${ }^{59}$ of using the term "Density Fitting" to refer to the general four-center integral handling described above, while "Resolution of the Identity" is reserved for more specific factorizations that are encountered in explicit correlation. A prefix "DF-" to a method's name can be used to indicate the use of density fitting, but is customarily dropped due to the high robustness and negligible errors in optimized implementations when calculating molecular properties and association energies.

\section{Local Electron Correlation}

Electron correlation in wavefunction-based quantum chemical calculations is introduced by "exciting" electrons from occupied into virtual orbitals of a reference wavefunction, and mixing the resulting corrections into the reference energy. This is usually done in a canonical picture where the occupied and virtual orbitals are taken directly from the preceding Hartree-Fock calculation. However, these symmetry-adapted orbitals may in general be delocalized over the entire molecular system, while the correlation energy itself is known to strongly depend on the overlap of the involved orbitals. ${ }^{60}$ As a result, an unfortunate number of significant, but "unspecific" correlation contributions must be computed. It is thus rewarding to localize the orbital space beforehand and preselect the theoretical rigor at which individual orbital pair contributions are treated on grounds of their spatial separation. ${ }^{61,62}$ A detailed account on such local correlation methods (indicated by a prefix " $L$ ") and their advantages is available in a comprehensive review by Korona et al. ${ }^{60}$ which is reproduced here in short.

Local correlation approaches allow significant computational savings by demoting a large number of less-relevant electron excitations to simpler computational levels, or even discarding them altogether. As an additional benefit, this largely eliminates the notorious basis-set superposition error $(\mathrm{BSSE})^{60,63}$ which is otherwise usually taken care of by counterpoise (CP) calculations at additional computational effort. ${ }^{64}$ Apart from more accurate electronic energy predictions, e.g. for internal conformational preferences $^{65}$ or intermolecular binding, harmonic vibrational frequencies have been shown to profit from the reduced BSSE. ${ }^{66,67}$ The accuracy gain of local over canonical methods seems to diminish for anharmonic vibrational treatments, ${ }^{68}$ but their performance advantages still uphold.

Looking at the drawbacks, local correlation methods are not as much of "black box" character as their canonical counterparts. First, the choice 
of localization method for the orbital space is not rigorously dictated, and different schemes may be applied. As it was found out, Pipek-Mezey localization $^{69}$ generally provides a robust description of the localized orbital space for most applications. ${ }^{70,71}$ In the established approach by Werner and co-workers which was employed throughout this work, the localized orbitals are subject to a population analysis to form "domains" of orbitals which represent localized electron pairs. ${ }^{71}$ While this provides a chemically intuitive picture of the molecular structure, it requires artificial charge cutoffs to be defined for building the domains. At ill-defined values, extensive mixing of low-charge contributions from distant orbital centers may bloat the domains and counteract the localization approach. ${ }^{\ddagger}$ Additional empirical distance thresholds must be introduced if long-range domain pairs are to be treated at a less rigorous level of theory than closer ones. Further, the localization procedure may be ambiguous in aromatic $\pi$ systems, or become inconsistent when sampling molecular structures along a wide range of internal coordinates. ${ }^{71,72}$ Freezing and merging procedures have been introduced for such cases to avoid discontinuities in the domain definitions (and thus the resulting energies) and correctly represent the underlying electronic structure in a localized picture. Still, the special care that such situations demand may exceed the scope of a wider user base.

The domain-localization approach allows to go even further than to only hierarchize the correlation treatment on grounds of distance aspects. In the LMOMO scheme, ${ }^{73}$ specific domains can be singled out to be treated at a certain level of theory while all others are reduced to some less-demanding default method. Changes in the chemical environment of some functional group can thus be treated in a strongly localized sense with little interference from the remainder of the system. Evidently, the degree to which the interactions are indeed localized in the selected domains determines the agreement with a full-scale treatment at the respective level of theory. Apart from the obvious computational savings, the LMOMO method can provide detailed insights into the quantum description of molecular systems, as it has been demonstrated for the methanol dimer. ${ }^{15}$

Combination with Density Fitting and explicit correlation (see below) has allowed the development of highly accurate local Møller-Plesset and Coupled Cluster correlation treatments, allowing down to linear scaling of the computational cost with the system $\operatorname{size}^{74}$ and extending their applicability to very large systems. ${ }^{75}$ As in the original suggestion by Saeb $\varnothing$ and Pulay, ${ }^{62}$ the virtual orbital space-which is less rigorously defined in a physical sense-

\footnotetext{
¥While generally unwanted, this effect was deliberately exploited herein to simulate canonical calculations in a local correlation formalism; see Appendix C for details.
} 
can be localized by using projected atomic orbitals (PAOs), which is also the approach undertaken in all local calculations within this work. Recently, the group of Neese, developing the ORCA program system, ${ }^{76}$ has devised an alternative approach based on Pair Natural Orbitals (PNOs) for the virtual space, ${ }^{77,78}$ spawning the first Coupled Cluster calculation on an entire protein of more than 600 atoms. ${ }^{79}$ Exploiting the sparsity of localized multielectron integral matrices promises further computational savings. ${ }^{80}$ Overall, it can be hoped that the physically justifiable and chemically intuitive concept of localizing electron correlation effects will advance the application of accurate quantum chemical methods throughout the chemical sciences.

\section{Explicit Correlation}

Electronic wavefunctions exhibit "cusps" for any two electrons approaching each other, reflecting their repulsive Coulomb interaction. ${ }^{81}$ However, the starting point for common ab initio methods is a Hartree-Fock calculation which, by construction, replaces the exact electron-electron interactions by a mean-field approximation. The correlation energy is then recovered based on excitations in the one-electron orbitals of the reference wavefunction, but these are not efficient to fully recover the Coulomb cusps. ${ }^{82}$ It is thus useful to model the correlation cusps in a more dedicated way during the calculations, which is done in "explicitly correlated" methods by Kutzelnigg and Klopper. ${ }^{83}$ The improved representation of the electronic wavefunction drastically reduces basis set incompleteness effects and allows to approach the method's complete basis set (CBS) limit much faster than in conventional calculations. Reviews of explicit correlation techniques have been given by Werner et al. ${ }^{82}$ and Klopper et al. ${ }^{59}$ from various perspectives and shall be reproduced here in brief.

In conventional correlation approaches, the interactions of two-electron "pair functions" with the virtual space play a leading role in the correlation energy. These pair functions are built as products $\phi_{i} \phi_{j}$ of one-electron orbitals from the reference wavefunction. In explicit correlation, their role is taken by "geminals" which additionally include some functional dependence on the inter-electron distance $r_{12}$. In the original formulation by Kutzelnigg and Klopper, ${ }^{83}$ a simple linear $r_{12}$ term was used; however, the most rewarding approaches have been found to be of exponential forms $\exp \left(-\gamma r_{12}\right)$ as first proposed by Ten-no. ${ }^{84}$ Historically, methods with a linear $r_{12}$ factor obtain a "-R12" suffix to their name, while any other form is denoted "-F12".

The conventional pair functions are, by virtue of the Hartree-Fock reference, inherently orthogonal to the rest of the wavefunction. To achieve the same for the geminals, projection operators must be applied before the cor- 
relation energies can be computed. Unfortunately, these projections produce three- and four-electron integrals in the correlation terms which are computationally difficult to approach. One technique to circumvent this problem is the approximative "CABS" (Complementary Auxiliary Basis Set) approach ${ }^{85}$ which uses a resolution-of-the-identity (RI) expansion of the wavefunction in an auxiliary basis set. This enables factorization of the offending integrals into two-electron forms, which are then significantly easier to handle. Corrections from the CABS treatment can further be patched into the HartreeFock reference energy, bringing it close to the CBS limit. ${ }^{82}$ For these reasons, CABS expansions today assume an important role for the projectors, and auxiliary "OPTRI" basis sets have been developed ${ }^{86}$ to accompany the dedicated explicit-correlation basis sets for the atomic orbitals. ${ }^{87}$

As noted previously, the RI method mentioned above must not be confused with Density Fitting (DF) which is commonly also called RI as well by some authors. Drawing a distinction between both approaches is of particular importance since DF expansions have been introduced to explicitly correlated methods as well in order to further improve their performance, in which case one auxiliary basis set must be provided for each expansion. Furthermore, the group of Werner ${ }^{74,82,88}$ has combined explicit and local correlation approaches, and their overall accuracy and performance make them highly attractive for application to large systems. In the context of Coupled Cluster theory, additional approximations must be introduced for calculating the excitation amplitudes. Throughout this work, the "-F12a" method by Adler et al. ${ }^{89,90}$ was adopted which provides some fortuitous and systematic error compensation in the correlation energies with small basis sets. $^{74,82}$ The perturbative triples included in CCSD(T)-F12 are not directly affected by explicit correlation, but one can include an approximative effect by scaling them according to the ratio of MP2 and MP2-F12 correlation energies, yielding "scaled triples" $\left(\mathrm{T}^{*}\right):^{90}$

$$
E\left(\mathrm{~T}^{*}\right)=E(\mathrm{~T}) \cdot \frac{E_{\mathrm{MP} 2-\mathrm{F} 12}}{E_{\mathrm{MP} 2}},
$$

This approximation was employed in all explicitly correlated CCSD(T) calculations throughout this work, and details on the practical implementation are given in Appendix C.

\subsubsection{Anharmonic Calculations}

\section{VSCF and Post-VSCF Methods}

The harmonic approximation represents the simplest starting point for the vibrational treatment of molecular systems. However, its restriction to de- 
coupled vibrational modes and purely quadratic potential functions leaves a wide gap to anharmonic reality. Closing this gap on a rigorous quantum mechanical basis requires to solve the vibrational Schrödinger equation in a higher-dimensional formulation, but this is again prohibited by the manybody problem. The strategies that have been developed to tackle this problem are in large parts analogous to those employed in electronic structure theories.

A first approximative solution to the Schrödinger equation is possible in a variational, self-consistent VSCF ("Vibrational SCF") picture. ${ }^{91-93}$ In this approach, the overall wavefunction of a system with $N$ vibrational modes remains separable as a product of $N$ single-mode functions. These so-called "modals" are coupled by a full $N$-dimensional potential, and the product ansatz allows to integrate out all but one of the coordinates. The remaining one-dimensional eigenproblem is solved in this effective potential, and the process is cycled through all modes until self-consistency is reached.

In principle, a full potential energy hypersurface in all internal degrees of freedom is required for the evaluation of the VSCF potential term. Creation of such a surface entails a large number of electronic energy calculations throughout the nuclear configuration space, which can obviously become prohibitively costly. The subsequent evaluation of the multidimensional potential integrals during the VSCF procedure poses another computational difficulty. Expanding the potential in a polynomial series provides some benefits in that only one-dimensional integrals have to be evaluated, and the task of calculating the potential hypersurface can likewise be broken down to a number of lower-dimensional subsurfaces. Still, this approach suffers from slow convergence of the solutions with respect to the polynomial order, especially in highly anharmonic, "floppy" systems. ${ }^{92,93}$ The choice of coordinates for the power expansion and VSCF calculation is often non-trivial as well and by far not solved exhaustively through normal coordinates. Alternatively, the potential term of the vibrational Schrödinger equation can be subdivided into individual $n$-mode contributions without the need for a polynomial representation. ${ }^{93}$ While this "multimode" approach by Bowman and co-workers further reduces the computational effort if low-dimensional contributions provide sufficient accuracy, it necessitates numerical techniques and still represents an effortful problem for anything but small molecules.

The anharmonicity effects contained in the VSCF solutions are largely implicit and approximate, since the true inter-mode couplings have been integrated out of the potential. Recovering these effects more accurately can be done by correlation approaches akin to the Møller-Plesset (VMP), Coupled Cluster (VCC) and Configuration Interaction (VCI) techniques found in electronic structure theory: ${ }^{92-94}$ by coupling different modals in the vibra- 
tional potential, energy corrections to the reference VSCF eigenstates are obtained. For a more in-depth view of VSCF and post-VSCF methods, a review by Gerber and Jung in Reference 92 is recommended.

\section{VPT2}

As an alternative to anharmonic (post-)VSCF calculations, the VPT2 method ("Vibrational Perturbation Theory to $2^{\text {nd }}$ Order") 95,96 provides estimates for anharmonic effects while avoiding the computational burden of building a full potential surface. Instead, a number of Hessian matrices at deflected geometries suffices to obtain the desired higher energy derivatives, and the vibrational Hamiltonian $\hat{H}$ is expanded as a Taylor series in the vibrational coordinates: ${ }^{95}$

$$
\begin{aligned}
\hat{H} & =\hat{H}^{0}+\hat{H}^{1}+\hat{H}^{2} \\
& =\frac{1}{2} \sum_{i} \omega_{i}\left(p_{i}^{2}+q_{i}^{2}\right)+\frac{1}{6} \sum_{i j k} \phi_{i j k} q_{i} q_{j} q_{k} \\
& +\frac{1}{24} \sum_{i j k l} \phi_{i j k l} q_{i} q_{j} q_{k} q_{l}+\sum_{\alpha} B_{\alpha}^{e} j_{\alpha}^{2}
\end{aligned}
$$

Here, $\omega_{i}, q_{i}$ and $p_{i}$ are the harmonic wavenumber, mass-weighted normal coordinate and associated momentum of mode $i$, respectively; the $\phi$ are the mass-weighted force constants-i.e., derivatives of the vibrational potential; and the last sum holds the equilibrium rotational constants $B_{\alpha}^{e}$ and vibrational angular momenta $j_{\alpha}$ around the rotational axes $\alpha$. Obviously, the term $\hat{H}^{0}$ represents the unperturbed harmonic problem, and anharmonic corrections come in from the third- and fourth-order terms contributed by $\hat{H}^{1}$ and $\hat{H}^{2}$, respectively (the latter further including the trailing rovibrational term). A perturbational calculation then results in the term expressions as given in Equation (1.1). As with all perturbation methods, however, this approach relies on the assumption that the anharmonic perturbation of the harmonic vibrational modes is relatively small. Significant anharmonic corrections to low-frequency motions can become problematic, possibly aggravated by inadequacies of the underlying electronic structure methods at treating shallow vibrational potentials.

\subsubsection{Software Packages and Basis Sets}

All calculations in this work were carried out using the GAUSSIAN $09^{97}$ and MOLPRO $2012^{98}$ program packages. While the former implements the efficient anharmonic VPT2 method, ${ }^{95,96}$ the latter software package was used for its implementation of efficient local and explicit correlation techniques. 
Dunning's correlation-consistent (aug-)cc-pVnZ basis sets were used throughout for the expansion of the atomic orbitals. Adhering to common practice, these basis sets will be abbreviated aVnZ. Diffuse functions on hydrogen atoms can often be dropped without significant impact on the accuracy, ${ }^{99}$ and such partially augmented basis sets are indicated by aug'-cc$\mathrm{pVnZ}\left(\mathrm{a}^{\prime} \mathrm{V} n \mathrm{Z}\right)$. However, spectroscopic properties of hydrogen-bonded structures may be sensitive to these types of basis functions. The present work thus also investigates the effects of selectively adding diffuse functions only to hydrogen atoms partaking in non-covalent contacts, and the resulting basis sets are called "selectively augmented", abbreviated saug-cc-pVnZ $(\mathrm{sVnZ})$. Since these augmentation patterns are somewhat arbitrary, they need to explicitly defined for each application, and their impact should be checked thoroughly against the corresponding partially $\left(\mathrm{a}^{\prime} \mathrm{V} n \mathrm{Z}\right)$ and fully $(\mathrm{aVnZ})$ augmented basis sets.

Besides the $a b$ initio methods used throughout this work, some B2PLYP and B3LYP calculations were conducted in GAUSSIAN 09 for more difficult and resource-demanding approaches, mostly anharmonic calculations. In all of these calculations, an ultrafine integration grid and tight geometry optimization criteria were invoked using the INT=ULTRAFINE and OPT=TIGHT options, respectively. All calculations include Grimme's D3 dispersion (D3), ${ }^{100}$ but spare Becke-Johnson damping due to implementation faults of analytic second derivatives in the D.01 revision of GAUSSIAN 09 (see below).

Density Fitting approximations were employed throughout in all MOLPRO calculations, using aVnZ/JKFIT ${ }^{56}$ auxiliary basis sets for Hartree-Fock Coulomb and exchange interactions and aVnZ/MP2FIT ${ }^{101}$ for MP2 and Coupled Cluster correlation energies. The "DF" prefix to the methods will be dropped throughout this work on grounds of the negligible errors introduced by the approximation. In explicitly correlated calculations, the VnZ-F12 AO basis sets by Peterson and co-workers ${ }^{87}$ were used in conjunction with the appropriate $\mathrm{aVnZ} / \mathrm{OPTRI}^{86}$ sets for the RI procedures.

Local Coupled Cluster calculations pose an additional problem when applied to intermolecular interactions due to their implementation in MOLPRO. Based on the default distance cutoffs for the classification of orbital pairs, any excitations across distinct molecules tend to be included only at a lower level of theory, typically MP2. This can be prevented by an INTERACT=1 command, which deliberately promotes all intermolecular orbital pairs to the highest level of theory irrespective of the distance criteria. This is indicated throughout by a suffix "int" to the method descriptors, and will be investigated further in the context of the systems studied herein.

Details on further input parameters for the MOLPRO calculations presented in this work can be found in Appendix C. 


\section{VPT2 Implementation in GAUSSIAN 09}

Several practical issues were discovered in the VPT2 implementation in GAUSSIAN 09, Revision D.01 (although possibly also present in other versions), which are discussed in Reference 17. Due to program bugs in Rev. D.01, analytic second derivatives are potentially unreliable when using Becke-Johnson damping for Grimme's D3 dispersion correction. ${ }^{102}$ Consequentially, all DFT-D3 results presented in this thesis were obtained with only zero-damping. Furthermore, the VPT2 results also exhibit a high sensitivity to the underlying molecular structure when combined with DFT methods, which leads to considerable variations in some band positions and anharmonicity constants upon varying the input structure to the preceding geometry optimization. Further scrutiny of the outputs revealed that this inconsistency lies in the Coriolis coupling contributions. Meanwhile, MP2 was found to be free of this instability, which suggests problems in the numeric DFT integration steps.

Beyond these DFT-specific inconsistencies, another problem was uncovered relating to the "checkpoint" files that are used by the program to store and access key results from the calculations. The VPT2 results were found to differ depending on whether the working molecular structure was used directly from a preceding geometry optimization, or retrieved from the checkpoint file of a separate calculation; this problem also impacts MP2 calculations. The effects are highly reproducible and hint towards possible accuracy errors in the storage of the optimized structural data.

In any way, stability and plausibility checks are necessary to extract useful estimates from DFT VPT2 calculations. Such a range of calculations has been presented in the Supplementary Information of Reference 17, and the results are reproduced in Appendix D.

Due to the large number of finite displacements, together with the perturbational approach, it was found to be numerically beneficial for VPT2 calculations to be conducted using basis sets without diffuse functions. ${ }^{103}$ This strategy was adopted throughout for the present work. 


\section{Chapter 2}

\section{Vibrational Dynamics in Methanol Monomers and Dimers}

Methanol represents the simplest aliphatic alcohol, offering a single hydroxy functionality for hydrogen bonding. The $\mathrm{O}-\mathrm{H}$ stretching motion is, in a coarse picture, decoupled from motions in the remainder of the molecule. However, the internal counter-rotation of the hydroxy and methyl groups experiences a small, threefold barrier, and the resulting tunneling effects have a significant impact on the structure of the $\mathrm{OH}$ stretching band. Although this torsion-stretching interaction is suppressed somewhat in alcohols with longer alkyl backbones due to the increased moment of inertia and decreased symmetry, they may exhibit a conformational variety which complicates studies of their aggregated forms. This comes into play already for ethanol, where the distinguishable trans and gauche monomer conformations allow for a total of up to 6 stable dimer structures with partially overlapping infrared bands. ${ }^{104,105}$ Again, methanol as a model system is advantageous in that it only forms a single detectable dimer structure. ${ }^{1,106,107}$

While the fundamental transition of the donor $\mathrm{OH}$ stretching motion in the methanol dimer (Figure 2.1) is well-established from an experimental perspective, other important vibrational motions have gone uncharacterized for a long time. This includes the librational motion, i.e. the torsion of the $\mathrm{OH}$ group in the donor molecule, which is transformed from the low-barrier, highly symmetric monomer situation into a much more confined and localized motion in a hydrogen bond. Moreover, information on the anharmonicity content of the stretching and libration motions, both in terms of their diagonal anharmonicity constants and mutual coupling, is difficult to obtain, since this demands the detection of overtone and combination transitions 


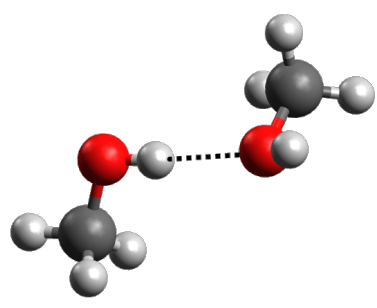

Figure 2.1: Structure of the methanol dimer.

in both modes. Only recently have the donor $\mathrm{OH}$ stretching overtone, ${ }^{14}$ the libration fundamental, ${ }^{103}$ and their combination band ${ }^{17}$ been observed in supersonic expansions and cryogenic matrix environments, providing access to some important anharmonicity constants for the dimer.

Approaching the stretching-libration dynamics in the methanol dimer from a theoretical side poses further difficulties, arising from the finding that many popular methods fail in quantitatively predicting the harmonic $\mathrm{OH}$ stretching red shift upon dimerization. ${ }^{15}$ Since this quantity is the most basic constituent in the experimentally observable dimerization shift, its accurate assessment is indispensable for a thorough quantitative analysis. Only with advanced computational methods, reliable predictions appear to be possible in this regard. Beyond the harmonic picture, variational anharmonic approaches to the methanol dimer remain problematic due to the large number of vibrational degrees of freedom, while the less demanding full-dimensional perturbational VPT2 approach has been found to systematically misestimate anharmonicity constants involving the librational motion. ${ }^{17}$ Furthermore, the substantial torsional tunneling effects in the methanol monomer are not honored by this type of calculations, since they only sample a single minimum of its threefold torsional potential.

In this chapter, the monomer reference will be discussed with respect to the $\mathrm{OH}$ stretching wavenumber and torsional tunneling effects, and attempts at a localization in the torsional subspace are discussed. Then, the experimental data from jet- and matrix-FTIR measurements for diagonal anharmonicity and the stretching-libration coupling are presented, followed by harmonic and anharmonic quantum chemical predictions. Lastly, the final section will attempt to explain the overall $\mathrm{OH}$ dimerization shift on grounds of the gathered data. 


\subsection{Torsion-Stretching Dynamics in Methanol Monomers}

Based on the rotational symmetry of the methyl group, the $\mathrm{OH}$ proton in the methanol monomer experiences a threefold potential for the torsional motion around the $\mathrm{C}-\mathrm{O}$ bond, with a rather low barrier on the order of $400 \mathrm{~cm}^{-1} .108$ Consequentially, pronounced tunneling interactions between the neighboring potential wells exist, splitting the otherwise triply-degenerate bound energy levels into states of A and E symmetry. This torsional behavior has been intensively studied (see, e.g., References 109, 108, 110, 111), together with the overall rotation of the molecular frame around the (pseudo-)figure $a$ axis that strongly interacts with it. ${ }^{112-114}$ In light of the cold conditions prevalent in supersonic expansions, and to facilitate comparison with simple theoretical models, the following discussion will be restricted to the rotational ground state where interactions with the torsional motion vanish. ${ }^{115,116}$ In addition, some resonances between rovibrational levels exist as detailed by Hunt et al. ${ }^{113}$ but are not considered to be relevant for the $J=0$ states investigated below. Throughout all following schemes, doubly degenerate levels will be shown as blue double lines, non-degenerate ones as red lines, and $v_{s}$ and $v_{t}$ denote the quantum numbers of the stretching and torsion modes, respectively.

Typical filet-jet FTIR spectra of the $\mathrm{OH}$ stretching fundamental and overtone bands are shown in Figure 2.2. Their intricate structure is partially lost to the low $2 \mathrm{~cm}^{-1}$ resolution in these spectra, but the overall expanse of the band systems due to residual rotational structure and offset $\mathrm{A} / \mathrm{E}$ band centers still becomes obvious.

Drawing from an extensive body of gas-phase data for the methanol monomer, ${ }^{108,113,117}$ some relevant stretching-torsion energy levels are assembled in Table 2.1 and on the left side of Figure 2.3 for torsion and stretching states each up to $\mathrm{v}=2$. Using Equation (1.6), the $\left(\mathrm{v}_{\mathrm{s}}, \mathrm{v}_{\mathrm{t}}\right)=(1,1) \leftarrow(0,0)$, $(1,0) \leftarrow(0,0)$ and $(0,1) \leftarrow(0,0)$ transitions yield stretching-torsion coupling constants of $x_{\mathrm{s}, \mathrm{t}}=+0.5 \mathrm{~cm}^{-1}$ for the A states and $+13.7 \mathrm{~cm}^{-1}$ for the E states. The analysis can be extended to the stretching overtones per Equations (1.3) and (1.4), with

$$
x_{\mathrm{s}, \mathrm{t}}=\frac{1}{2}\left[\tilde{v}^{(2,1) \leftarrow(0,0)}-(2 \tilde{v})_{\mathrm{s}}-\tilde{v}_{\mathrm{t}}\right],
$$

yielding very similar results of $x_{\mathrm{s}, \mathrm{t}}=+0.1 \mathrm{~cm}^{-1}$ and $+13.2 \mathrm{~cm}^{-1}$ for the A and $\mathrm{E}$ states, respectively.

The finite barrier height of the torsion potential implies that at sufficiently high excitation, the energy levels of the torsional motion will resem- 


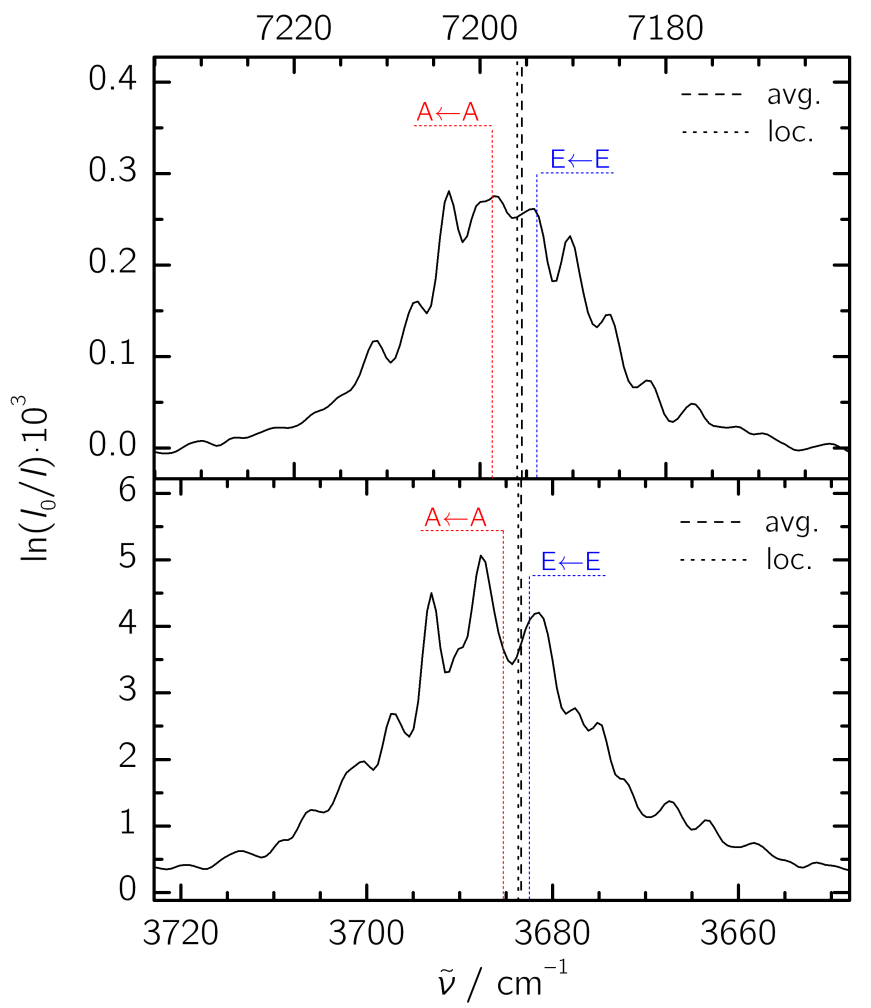

Figure 2.2: $\mathrm{OH}$ fundamental (bottom) and overtone (top) stretching bands of the methanol monomer in the filet jet (from Ref. 14, courtesy of F. Kollipost). Annotated are the band centers of $\mathrm{A} \leftarrow \mathrm{A}$ and $\mathrm{E} \leftarrow \mathrm{E}$, weighted-average ("avg."), and plateau-localized ("loc.") transitions; see Sec. 2.1.1 for details on the two latter schemes.

ble those of a free rotor rather than a bound particle. ${ }^{109}$ To model the torsionrotation problem, a Hamiltonian of the form ${ }^{118}$

$$
\hat{H}=F\left(j_{\alpha}+\rho J_{a}\right)^{2}+V_{\text {tors }}
$$

can be used, where $j_{\alpha}$ is the torsional momentum operator around the C$\mathrm{O}$ bond, and $\rho J_{a}$ introduces couplings to the rotational motion of the entire molecular frame projected onto the $a$ axis. Since the present treatment is concerned only with $J_{a}=0$ states, the latter term vanishes. As will be shown below, good agreement between theory and experiment is obtained in this formulation. The proportionality constant $F=\hbar /(4 \pi c \cdot I)$ contains the effective moment of inertia $I$ associated with the torsional motion. 


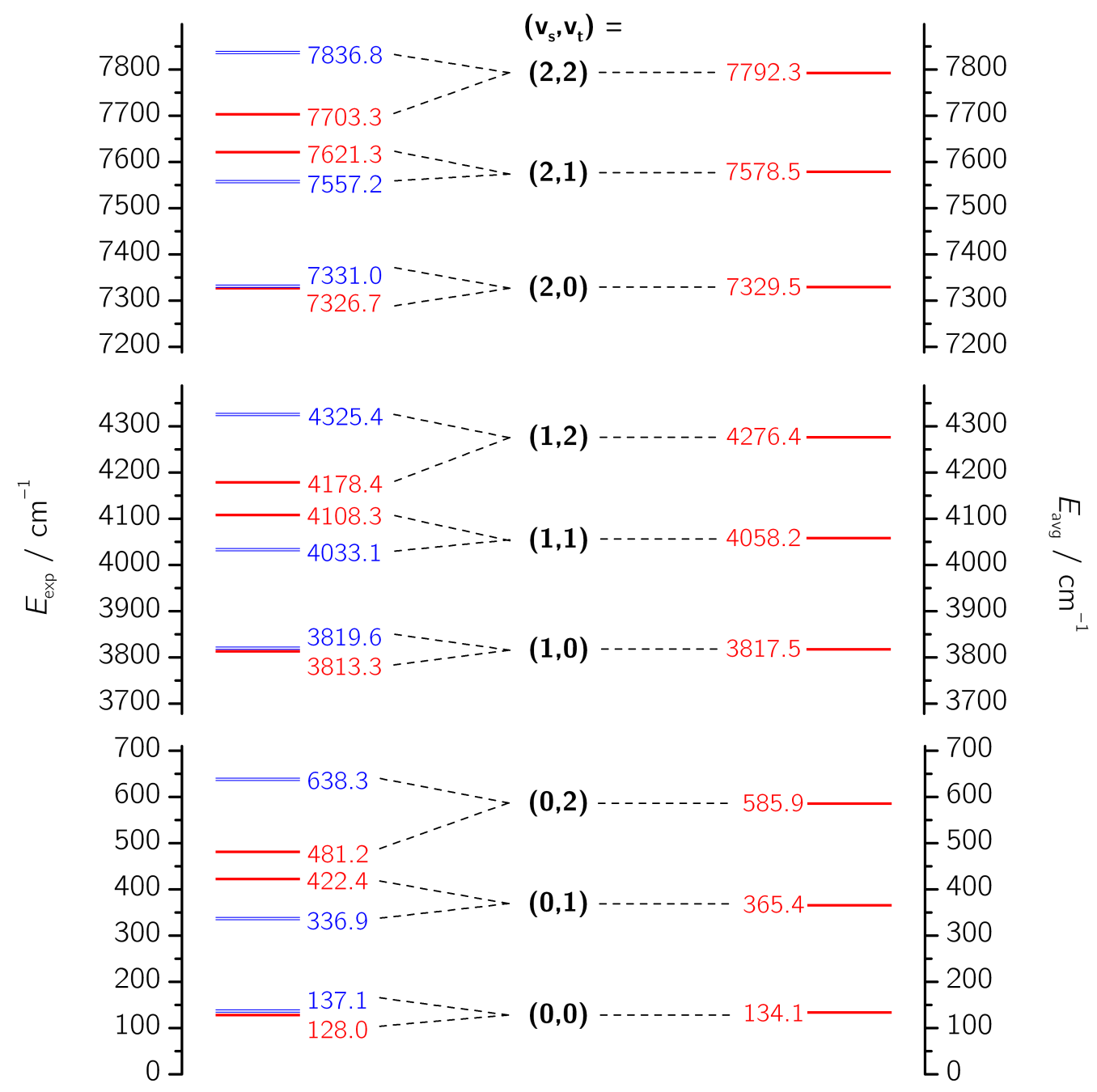

Figure 2.3: $\mathrm{OH}$ stretching-torsion energy levels of the methanol monomer up to $\left(\mathrm{v}_{\mathrm{s}}, \mathrm{v}_{\mathrm{t}}\right)=(2,2)$, with experimental data $\left(E_{\text {exp }}\right.$, Refs. $\left.108,113,117\right)$ on the left and weighted-average localized levels ( $E_{\text {avg }}$, see Sec. 2.1.1) on the right. 
Table 2.1: Torsion-stretching transitions (in $\mathrm{cm}^{-1}$ ) in the methanol monomer: Gas-phase experiment ("exp.") and weighted-average (“avg.") values for the stretching fundamental and first overtone.

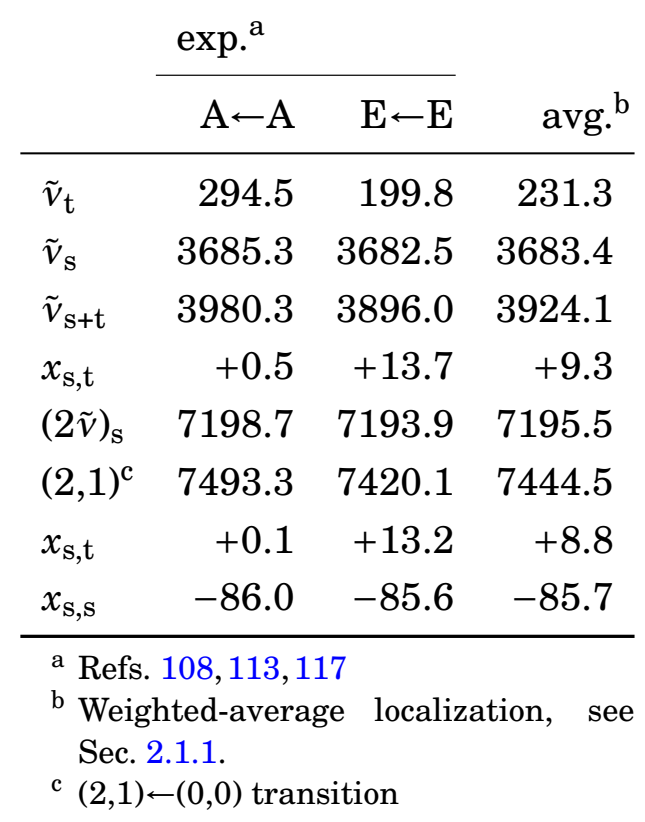

For the potential $V_{\text {tors }}$, a triple-minimum function of the form

$$
V_{\text {tors }}(\theta)=\frac{V_{3}}{2}[1-\cos 3 \theta]+\frac{V_{6}}{2}[1-\cos 6 \theta],
$$

is customarily used, with $\theta$ as the angular displacement of the $\mathrm{OH}$ group. The relevant parameters $F, V_{3}$, and $V_{6}$ have been determined by $\mathrm{Xu}$ and Hougen ${ }^{119}$ for the stretching ground state, Hunt et al. ${ }^{113}$ for its first excited state, and Rueda et al. ${ }^{108}$ for its second excited state.

\subsubsection{Torsional Localization Models}

The quantum chemical methods employed within this work sample only a small portion of a potential energy hypersurface around a single minimum in order to infer the vibrational properties of a system. For the methanol monomer, this is clearly in stark contrast to the true torsional motion which is dominated by the extensive tunneling interactions. In order to compare the predictions to experimental data, one thus needs to make these two drastically different situations compatible with each other. This problem can be 
approached from both sides: one can either introduce tunneling interactions to the "localized" theoretical predictions, or alternatively, reduce the "delocalized" experimental picture to an artificial single-minimum situation. It appears to be arguably easier to follow the latter approach and establish a localized monomer reference instead of subjecting each theoretical data set to an individual delocalization procedure. A localized reference will furthermore simplify the upcoming discussion of $\mathrm{OH}$ stretching dimerization shifts, since it lifts the ambiguities in the stretching band centers that are present in the torsionally delocalized monomer.

To this end, two localization models will be discussed below. The first one, reproduced here from Reference 17, is a state-specific approach that reduces each experimental $\mathrm{A} / \mathrm{E}$ state triplet to a triply-degenerate situation through a uniform coupling element. Due to the simple expressions that result for the localized state energies, this model will be called the "weighted-average" approach below. The second model assumes a new vibrational potential function that mimics a single torsional minimum, suspended from a potential energy "plateau". By using a set of numerical routines, variational eigenvalues have been obtained for these modified potentials and will be discussed below.

\section{Weighted-Average Model}

The first localization model assumes degenerate $\left(\mathrm{v}_{\mathrm{s}}, \mathrm{v}_{\mathrm{t}}\right)$ state triplets to be split by a uniform coupling element $w$ among the neighboring potential wells. ${ }^{17}$ From the eigenvalues of the corresponding $3 \times 3$ coupling matrices, the observable A-E splittings amount to $3 w$, and the localized energy levels represent the degeneracy-weighted average of the delocalized ones. The localized levels can thus be calculated in a straightforward manner from experimental data, and the results are displayed on the right-hand side of Fig-

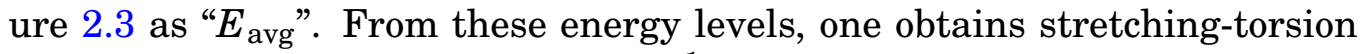
couplings of $x_{\mathrm{s}, \mathrm{t}}=+9.3$ and $+8.8 \mathrm{~cm}^{-1}$ for the stretching fundamental and overtone, respectively. At the same time, the diagonal $\mathrm{OH}$ stretching anharmonicity constant $x_{\mathrm{s}, \mathrm{s}}$ in the weighted-average model is $-85.7 \mathrm{~cm}^{-1}$. This value remains close to jet-experiment values of around $-86 \mathrm{~cm}^{-1}$, even when taking into account uncertainties from different approaches to locating the stretching band centers. ${ }^{14}$ The weighted-average band centers are indicated in Figure 2.2 by "avg." and listed in Table 2.1.

The eigenvalues of the torsional Hamiltonian given in Equation (2.2) (see Figure 2.3, left and Figure 2.4, left) place the $\mathrm{v}_{\mathrm{t}}=1 \mathrm{~A}$ sub-level just above the hindering potential barrier in the ground and first excited stretching states. Only for $\mathrm{v}_{\mathrm{s}}=2$ does this level become subtly confined due to the 
increasing barrier height. Furthermore, strong interactions with the adjacent $\mathrm{v}_{\mathrm{t}}=2 \mathrm{~A}$ state will likely push both levels apart. These effects are not appreciated by the simple state-averaging scheme, and sizable errors near the top of the hindering potential may result. Further above the barrier, the weighted-average approach even formally fails to capture the transition from the (near-)bound $\mathrm{A} / \mathrm{E}$ states to the doubly degenerate free-rotor levels.

\section{Plateau-Localization Model}

The alternative plateau-localization model replicates a single potential well from Equation (2.3) in the $\theta=[-\pi / 3,+\pi / 3]$ interval, and replaces the neighboring ones by a plateau equal to the barrier height (see Figure 2.4, right). These potential functions conceptually isolate the torsional motion in a single minimum while still allowing the eigenstates to become non-bonded above the respective plateau height. The eigenvalues thus approach the correct free-rotor behavior at higher excitations, save for a constant offset relative to the correct triple-well situation. Variational solutions to the onedimensional torsional Schrödinger equation were calculated with a homemade set of numerical MATLAB routines (see Appendix B for details), and their robustness was verified by modeling the eigenstates of the Hamiltonian in Equation (2.2) with the correct threefold potentials. The level energies and resulting transitions are reproduced to reasonable accuracy, with errors below $1 \mathrm{~cm}^{-1}$ (see Figures 2.3 and 2.4, and Table 2.1). The parameters employed in the present work are given in Appendix B, based on the concise overview provided by Rueda et al. in Reference 108 .

Using the plateau-localization scheme, the stretching band centers of the fundamental and overtone transitions uphold to within $0.5 \mathrm{~cm}^{-1}$ with respect to their weighted-average counterpart, and are indicated in Figure 2.2 by "loc." (see also Table 2.2). From both transitions, the plateau model yields a diagonal monomer stretching anharmonicity of $x_{\mathrm{s}, \mathrm{s}}=-85.1 \mathrm{~cm}^{-1}$, which is in good agreement with both the delocalized experimental and localized weighted-average values. At higher excitations, the plateau model by construction behaves correctly in gradually merging its non-degenerate eigenstates into rotational doublets.

\section{Localized Vibrational Dynamics}

In order to calculate localized stretching-torsion couplings, the most straightforward approach would be to use the two lowest torsional states within each stretching manifold, which yields a localized fundamental wavenumber of $212.0 \mathrm{~cm}^{-1}$ (column "lowest" in Table 2.2). However, one 


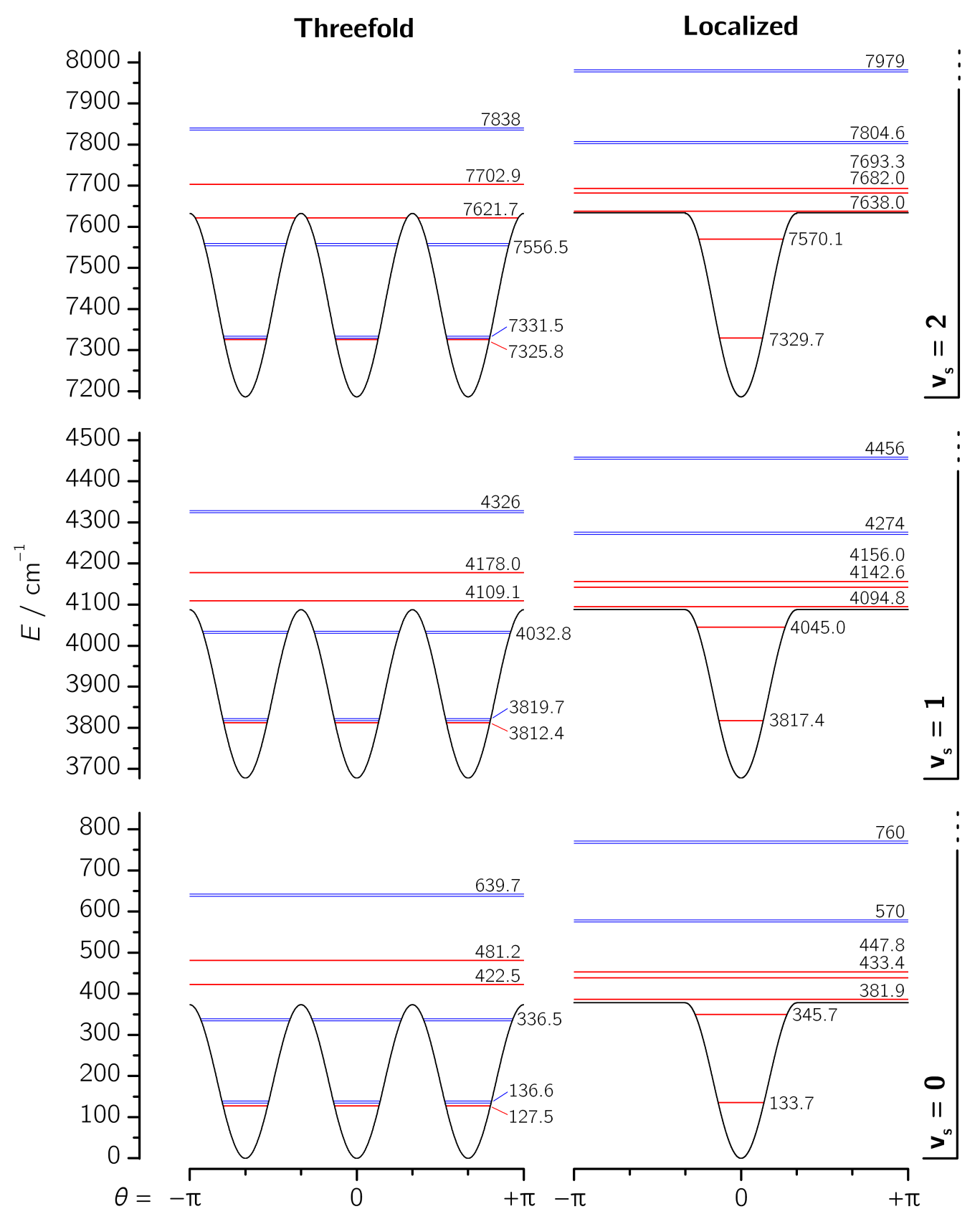

Figure 2.4: Calculated $\mathrm{OH}$ stretching-torsion energy levels of the methanol monomer up to $\mathrm{v}_{\mathrm{s}}=2$, using the correct threefold torsion potentials (from Eq. (2.3), left) and the corresponding plateau-localized models (right). 

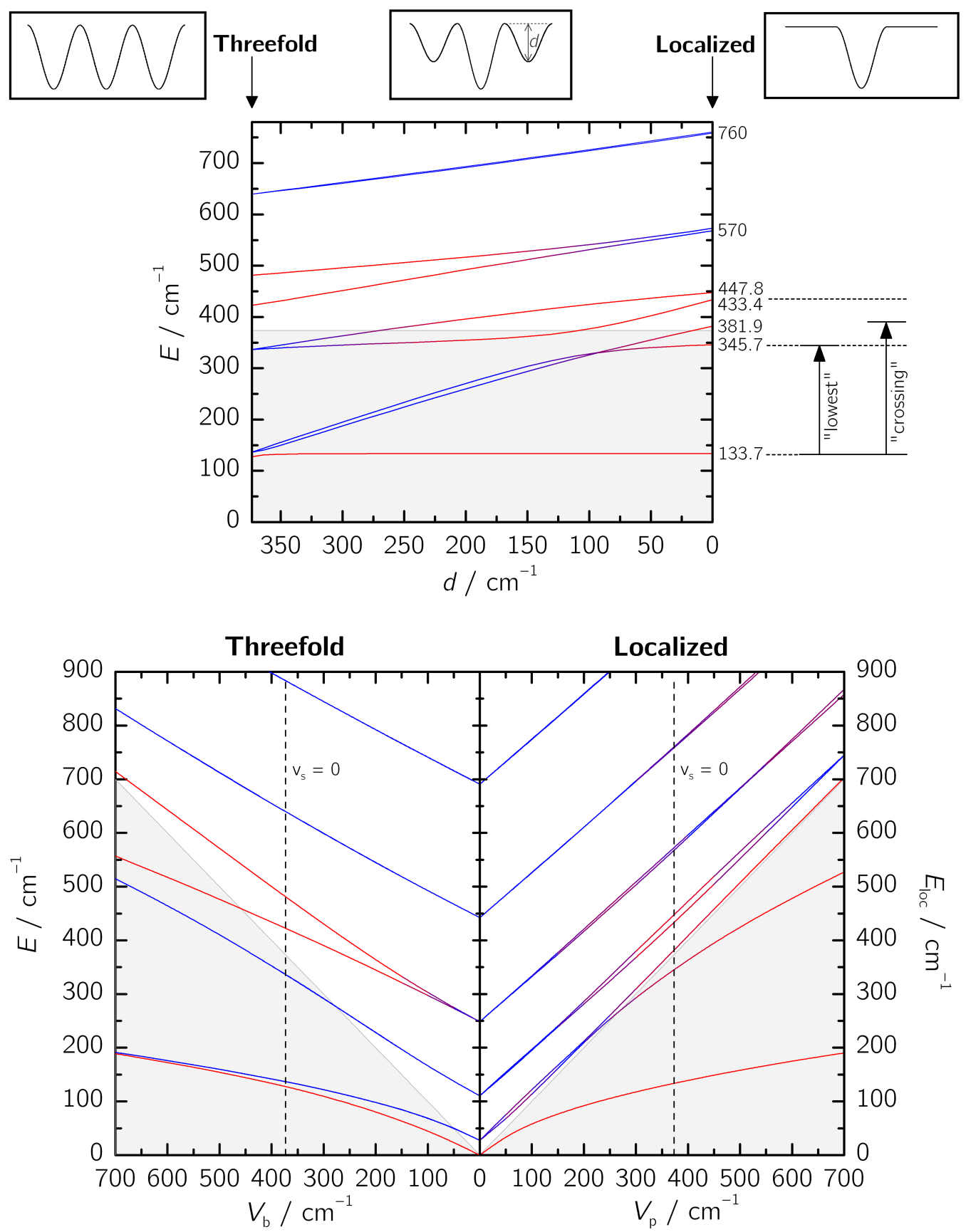

Figure 2.5: Evolution of torsional eigenstates upon varying the degree of localization (top panel) through the depth $d$ of the two side-wells for $\mathrm{v}_{\mathrm{S}}=0$; and upon varying the barrier/plateau heights $V_{\mathrm{b}}$ and $V_{\mathrm{p}}$ (bottom panel). Greyshaded areas represent energies below the barrier/plateau. The "lowest" and "crossing" transitions from Tab. 2.2 are visualized in the top figure, see the text for details. 
Table 2.2: Localized transitions and anharmonicity constants (in $\mathrm{cm}^{-1}$ ) in the methanol monomer: weighted-average experimental ("avg.", from Tab. 2.1) and plateau-localized ("loc.") values for the stretching fundamental and first overtone. The localized data are calculated using either the lowest excited torsional state ("lowest"), or the average of the states pertinent to an avoided crossing ("crossing"); see the text for details. All data in $\mathrm{cm}^{-1}$.

\begin{tabular}{lrrr}
\hline & \multicolumn{3}{l}{ loc. } \\
\cline { 3 - 4 } & avg. & lowest & crossing \\
\hline$\tilde{v}_{\mathrm{t}}$ & 231.3 & 212.0 & 255.9 \\
$\tilde{v}_{\mathrm{s}}$ & 3683.4 & 3683.7 & 3683.7 \\
$\tilde{v}_{\mathrm{s}+\mathrm{t}}$ & 3924.1 & 3911.3 & 3960.1 \\
$x_{\mathrm{s}, \mathrm{t}}$ & +9.3 & +15.6 & +20.6 \\
$(2 \tilde{v})_{\mathrm{s}}$ & 7195.5 & 7196.0 & 7196.0 \\
$(2,1)^{\mathrm{a}}$ & 7444.5 & 7436.4 & 7492.4 \\
$x_{\mathrm{s}, \mathrm{t}}$ & +8.8 & +14.2 & +20.3 \\
$x_{\mathrm{s}, \mathrm{s}}$ & -85.7 & -85.7 & -85.7 \\
\hline
\end{tabular}

a $(2,1) \leftarrow(0,0)$ transition

drawback of the plateau model is the intricate relationship between the localized energy levels and their correct threefold-degenerate pendants. Figure 2.5 demonstrates the evolution of the eigenstates between both situations by varying either the degree of localization $d$ (top panel), i.e. the depth of the two potential wells adjacent to the central one, or the barrier and plateau heights $\left(V_{\mathrm{b}}\right.$ and $V_{\mathrm{p}}$, resp.; bottom panel). Both evolution diagrams suggest that the second-lowest localized torsion state appears to owe its confined character mostly to an avoided crossing with another level further up, which only comes into effect for plateau heights above $c a .300 \mathrm{~cm}^{-1}$. The true methanol barriers are possibly still close enough to this onset point that the state in question still carries a somewhat mixed character in the fully plateau-localized calculations. In order to account for these ambiguities, one could argue for averaging both partaking levels, which yields a torsional fundamental wavenumber of $255.9 \mathrm{~cm}^{-1}$ in the stretching ground state (column "crossing" in Table 2.2, visualized in Figure 2.5). While still somewhat arbitrary, this result is reasonable in light of the weighted-average wavenumber of $231.3 \mathrm{~cm}^{-1}$. From all possible results listed in Table 2.2, 
an approximate localized transition wavenumber of $230(20) \mathrm{cm}^{-1}$ appears justified, but this value will retain its uncertainties unless a more physical description of the localized situation is proposed. The ambiguities furthermore carry forward to the stretching-torsion band, for which a wavenumber of about $3930(30) \mathrm{cm}^{-1}$ can be assumed.

More so than the fundamental transition, the first torsion overtone is illdefined from the level-evolution perspective. Here, the straightforward approach suggests that the final localized state for this transition in the $\mathrm{v}_{\mathrm{s}}=0$ manifold would be the $381.9 \mathrm{~cm}^{-1}$ level (see Figure 2.5, top) that barely escapes the potential well, which would imply a diagonal anharmonicity constant of $x_{\mathrm{t}, \mathrm{t}}=-87.9 \mathrm{~cm}^{-1}$. The corresponding $\mathrm{A}$ and $\mathrm{E}$ values in the threefold potential are -117.8 and $+50.8 \mathrm{~cm}^{-1}$, respectively, with a weighted average of $-5.4 \mathrm{~cm}^{-1}$. Clearly, the situation in the intermediate region between the confined and free-rotor limiting cases effectively prohibits a direct comparison of the localized and delocalized situations.

Concerning the desired stretching-torsion coupling constants, the straightforward lowest-level approach to the localized problem yields $x_{\mathrm{s}, \mathrm{t}}=$ $+15.6 \mathrm{~cm}^{-1}$ for the stretching fundamental, and $+14.2 \mathrm{~cm}^{-1}$ for its overtone. Averaging the two localized torsion states pertaining to the avoided crossing, as suggested above, yields values of +20.6 and $+20.3 \mathrm{~cm}^{-1}$ for the two stretching transitions (column "crossing" in Table 2.2). Together with the weighted-average values of +9.3 and $+8.8 \mathrm{~cm}^{-1}$, an overall localized stretching-torsion coupling of $x_{\mathrm{s}, \mathrm{t}}=+15(6) \mathrm{cm}^{-1}$ emerges. Fortunately, the stretching wavenumbers are less ambiguous due to the stronger confinement of the torsional ground states at the bottom of the potential wells and the consequentially smaller impact of the tunneling effects. Allowing for some uncertainties in the numerical solutions, the respective fundamental and overtone transitions can be placed at $3684(1)$ and $7196(2) \mathrm{cm}^{-1}$, yielding an anharmonicity constant of $x_{\mathrm{s}, \mathrm{s}}=-86(1) \mathrm{cm}^{-1}$.

Finally, it should be kept in mind throughout the remainder of this work that the localization approaches above do not carry direct physical meaning as to the vibrational dynamics of the methanol monomer. Rather, their purpose is to establish possible junction points with the quantum chemical calculations and their inherently local character.

\subsection{Jet- and Matrix-FTIR Spectroscopy}

As outlined in Section 1.1, a donor-OH stretching vibration in a hydrogen bond constitutes a sensitive probe for the interaction based on both the magnitude of its spectroscopic red shift and its intensity increase. According to 
Equation (1.3), diagonal and off-diagonal anharmonicity terms pertain to the observable wavenumber of a fundamental transition, added on top of a harmonic component. In order to calculate the dimerization shift, the dimermonomer differences in all contributions must be considered. Throughout the remainder of this work, "Dim" and "Mon" superscripts will therefore be used to provide the relevant context. The dimerization shift is hence given by $-\Delta \tilde{v}=\tilde{v}^{\text {Mon }}-\tilde{v}^{\text {Dim }}$, with the negative sign included for the convenience of writing the overall dominating red shifts as positive numbers. Drawing from Equation (1.3),

$$
-\Delta \tilde{v}_{\mathrm{s}}=-\Delta \omega_{\mathrm{s}}-2 \Delta x_{\mathrm{s}, \mathrm{s}}-\frac{1}{2} \sum_{i \neq \mathrm{s}} \Delta x_{\mathrm{s}, i}
$$

The different summation ranges for the monomer (11 off-diagonal terms) and the dimer (29 terms) are implied for all $\Delta x$ sums here and throughout. In addition, the stretching-torsion coupling in the monomer $\left(x_{\mathrm{s}, \mathrm{t}}^{\mathrm{Mon}}\right)$ and the corresponding coupling to the libration ("l") in the dimer $\left(x_{\mathrm{s}, 1}^{\mathrm{Dim}}\right)$ can be separated from the remaining terms, which will be indicated throughout by a primed summation sign, i.e.

$$
\sum_{i \neq \mathrm{s}} x_{\mathrm{s}, i}=\sum^{\prime} x_{\mathrm{s}, j}+x_{\mathrm{s}, 1 / \mathrm{t}}
$$

The primed summation index $j$ now excludes both the $\mathrm{OH}$ stretching and respective libration or torsion motions (" $1 / \mathrm{t}$ ", depending on whether one considers the dimer or monomer situation). Forming the dimer-monomer difference relevant to Equation (2.4) is straightforwards, yielding

$$
\sum_{i \neq \mathrm{s}} \Delta x_{\mathrm{s}, i}=\sum^{\prime} \Delta x_{\mathrm{s}, j}+\Delta x_{\mathrm{s}, 1 / \mathrm{t}}
$$

with $\Delta x_{\mathrm{s}, 1 / \mathrm{t}}=x_{\mathrm{s}, 1}^{\mathrm{Dim}}-x_{\mathrm{s}, \mathrm{t}}^{\mathrm{Mon}}$. The different remaining summation ranges for the dimer (28 terms) and the monomer (10 terms) are again implied.

\subsubsection{Filet-Jet Spectra}

Jet-FTIR spectra of pure methanol are shown in Figure 2.6, reproduced here in part from Reference 14. The wavenumber axis of the overtone spectrum is compressed by a factor of 2 and shifted by $171.4 \mathrm{~cm}^{-1}$ to match the localized monomer $\mathrm{OH}$ stretching band positions (see Table 2.2). This style of display encodes the change in diagonal anharmonicity, $\Delta x_{\mathrm{s}, \mathrm{s}}=x_{\mathrm{s}, \mathrm{s}}^{\mathrm{Dim}}-x_{\mathrm{s}, \mathrm{s}}^{\mathrm{Mon}}$, as a visible offset of the dimer overtone band relative to its corresponding fundamental.*

\footnotetext{
*Note that the size of this offset corresponds to $2 \Delta x_{\mathrm{s}, \mathrm{s}}$ if one interprets it in the compressed overtone scale itself.
} 


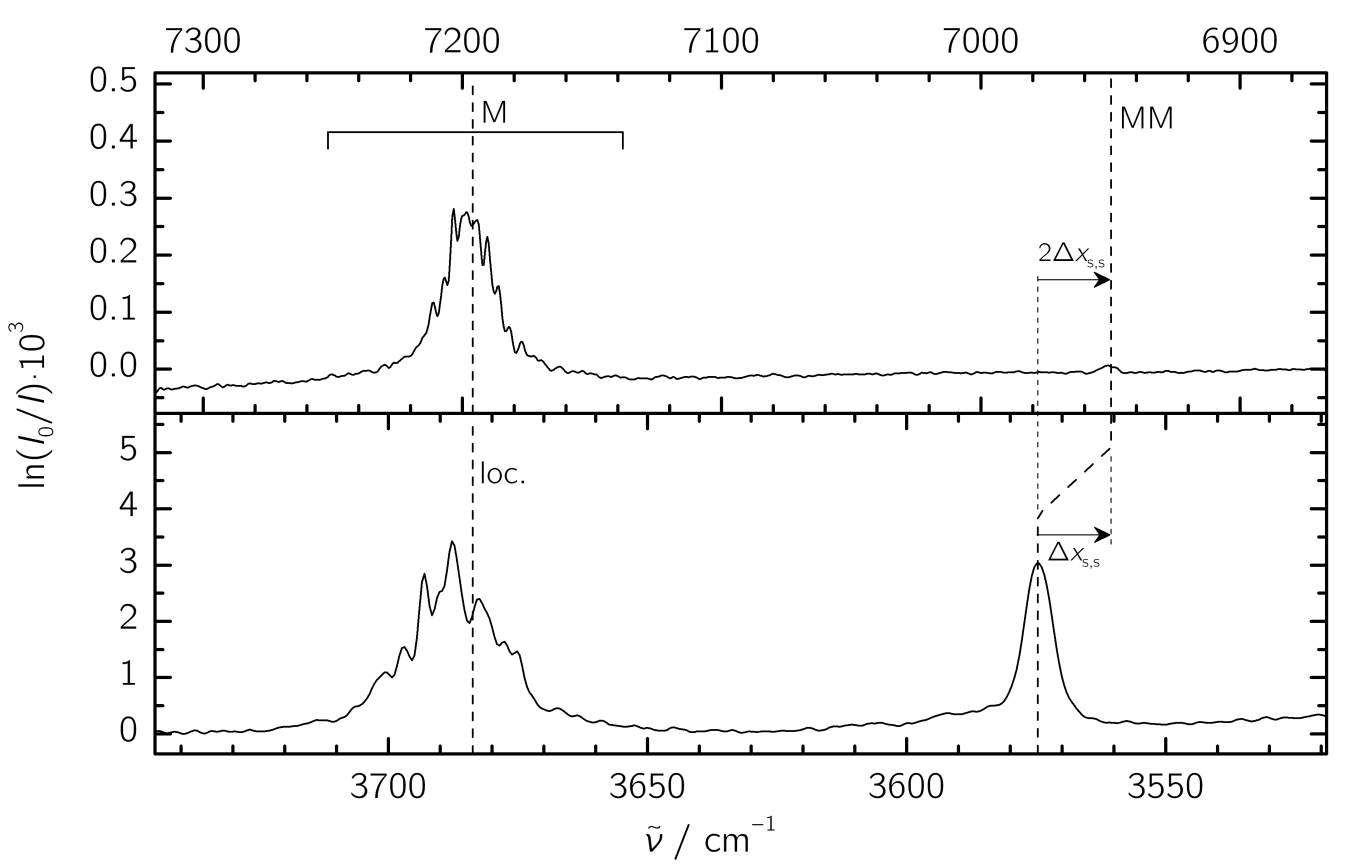

Figure 2.6: Jet-FTIR spectra of methanol in the $\mathrm{OH}$ stretching fundamental (bottom) and overtone (top) regions, courtesy of F. Kollipost (see also Ref. 14). The monomer ("M") and dimer ("MM") band centers are indicated by dashed lines, using the localized wavenumbers from Tab. 2.2.

As outlined above, the extensive tunneling and residual rotational band structure of the methanol monomer renders its stretching wavenumbers somewhat ambiguous. Kollipost et al. ${ }^{14}$ have used approximate values of 3686 and $7198 \mathrm{~cm}^{-1}$ for the fundamental and overtone bands, respectively, based on Raman and IR intensity maxima. These values imply a diagonal stretching anharmonicity content of $x_{\mathrm{S}, \mathrm{S}}^{\mathrm{Mon}}=-86 \mathrm{~cm}^{-1}$, but a $\pm 1 \mathrm{~cm}^{-1}$ error bar remains from the ambiguities in locating the band centers. Alternatively, one can make use of the localized transitions determined above (see Table 2.2), which approximately agree on the stretching anharmonicity of $x_{\mathrm{s}, \mathrm{S}}^{\mathrm{Mon}}=-85.7 \mathrm{~cm}^{-1}$ but place the band centers at slightly lower wavenumbers of about 3684 and $7196 \mathrm{~cm}^{-1}$.

In contrast to the monomer situation, the dimer stretching bands are well-defined due to the much smaller rotational constants and the absence of any significant tunneling structure. Based on the spectra in Figure 2.6, Kollipost et al. ${ }^{14}$ have found fundamental and overtone donor stretching wavenumbers of $3574.5(3)$ and $6950.6(6) \mathrm{cm}^{-1}$ and a resulting diagonal an- 
harmonicity constant of $x_{\mathrm{s}, \mathrm{s}}^{\mathrm{Dim}}=-99.2(4) \mathrm{cm}^{-1}$. This implies an increase in diagonal anharmonicity content by about $\Delta x_{\mathrm{s}, \mathrm{s}}=-13.5 \mathrm{~cm}^{-1}$ upon formation of the hydrogen bond. ${ }^{\dagger}$ Since this value is added twice in the calculation of a transition wavenumber, the diagonal anharmonic correction to the dimerization red shift amounts to $-2 \Delta x_{\mathrm{s}, \mathrm{s}}=27(2) \mathrm{cm}^{-1}$. The overall shift obviously depends on the chosen monomer reference, but the robust behavior of the stretching transitions under the different localization approaches presented in Section 2.1 suggests an overall value of $-\Delta \tilde{v}_{\mathrm{S}}=109(1) \mathrm{cm}^{-1}$.

Further information on the strength of the intermolecular interaction is contained in the intensity ratio of the fundamental and overtone dimer bands (see Section 1.1). From the spectra displayed in Figure 2.6, a ratio of 320(90) was determined by Kollipost et al. for the dimer, contrasted by a much smaller monomer value of 6(1) which may, however, be unreliable due to the higher peak extinction. ${ }^{14}$ Calculations suggest that most of these effects stem from a more than ten- to twenty-fold increase in the fundamental intensity from the monomer to the dimer, whereas the overtone intensity decreases only by a factor of about $5 .{ }^{14}$ These data provide a direct demonstration of the intensity impairments discussed in Section 1.1, and will serve as an experimental reference for quantum chemical predictions further below.

\subsubsection{Matrix-FTIR Spectra}

As in the monomer case, experimental assessment of the stretching-libration coupling $x_{\mathrm{s}, 1}^{\mathrm{Dim}}$ in the dimer requires the corresponding combination band wavenumber, $\tilde{v}_{\mathrm{s}+1}^{\mathrm{Dim}}$. Matrix isolation experiments were conducted to observe this presumably weak band, using the apparatus maintained by the group of Wugt Larsen at the MAX-lab facility in Lund, Sweden, and the results are reproduced here from Reference 17 . Figure 2.7 shows spectra of methanol dimers embedded in a neon matrix, sampled before (black) and after (red) annealing to $9 \mathrm{~K}$, with difference spectra ("diff.") in blue. In addition, $\mathrm{OH}$ libration fundamental spectra are included from an earlier study. ${ }^{103}$ Due to optical saturation, the intensities of the dimer stretching fundamental are unreliable, and the annealing difference trace is omitted for this band. Neon was specifically chosen over other common substrates since it typically induces lower perturbations in the vibrational signatures of the embedded guest molecules. ${ }^{120-122}$

\footnotetext{
${ }^{\dagger}$ Arguably, one might also speak of a decrease rather than an increase in anharmonicity due to the negative sign. More intuitively however, anharmonicity can be regarded as a departure from the harmonic behavior in either direction, which warrants this wording.
} 

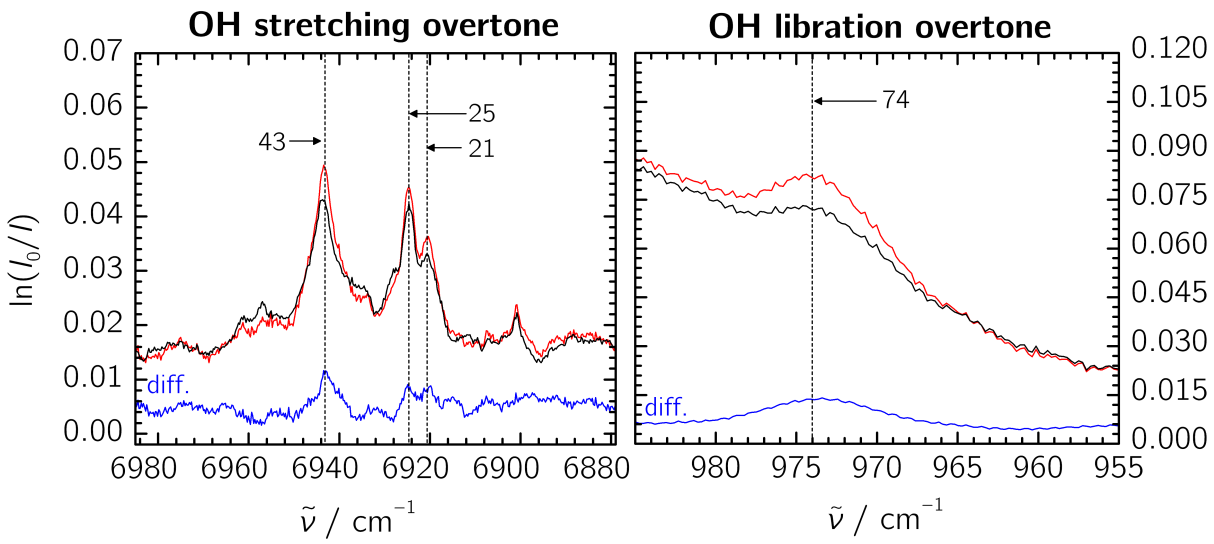

$\mathrm{OH}$ stretching-libration combination
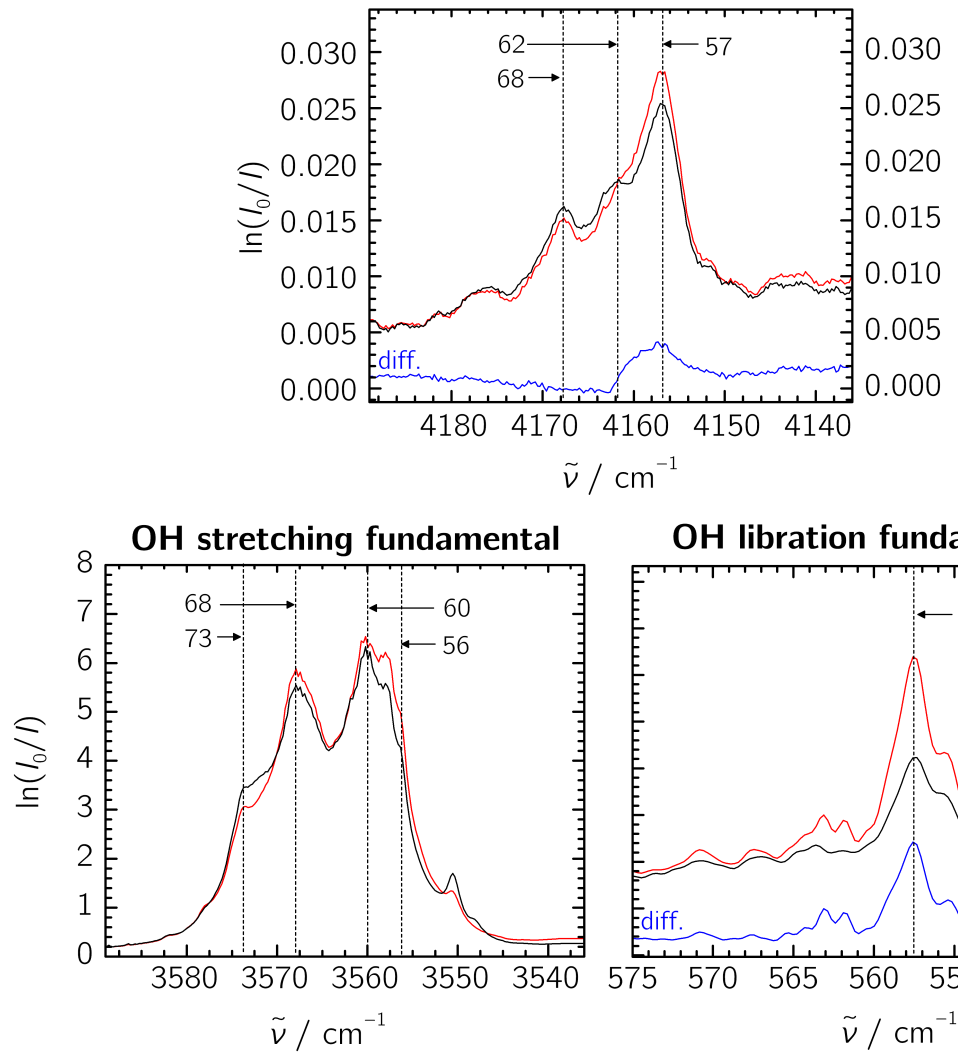

$\mathrm{OH}$ libration fundamental

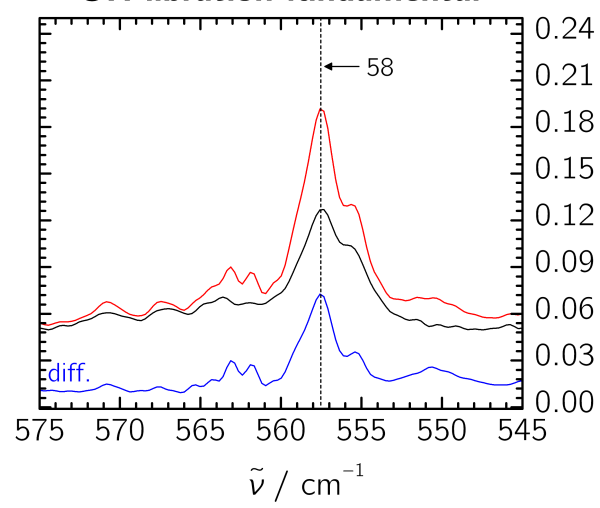

Figure 2.7: FTIR spectra of methanol dimers embedded in neon matrices (adapted from Ref. 17, courtesy of J. Andersen). Black and red traces show pre- and post-annealing spectra, respectively, with their differences in blue (omitted for the $\mathrm{OH}$ stretching fundamental due to optical saturation distortions). Annotated are the last two digits of sub-band wavenumbers in $\mathrm{cm}^{-1}$. 
Despite this usually benign behavior of neon as a matrix host, the donor $\mathrm{OH}$ stretching fundamental band displayed in Figure 2.7 is spread out over a region of about $30 \mathrm{~cm}^{-1}$, which is incompatible with the jet spectra (Figure 2.6). Furthermore, the complicated sub-band patterns that are visible in these spectra were found to be mostly insensitive to deuteration of the methyl group, but change when the $\mathrm{OH}$ proton itself is replaced by deuterium. ${ }^{17}$ This behavior is akin to tunneling of the $\mathrm{OH}$ proton into different potential minima, but any such effect should be quenched by the highly directional hydrogen bond in the dimer at least for the donor $\mathrm{OH}$ torsion. Despite their yet unknown origin, an identification of several sub-bands was attempted. These assignments are listed in Table 2.3, and the last two digits of the respective wavenumbers are annotated in Figure 2.7.

Based on previously assigned monomer transitions by Perchard ${ }^{114}$ and the observed annealing trends, a band formation around $4150-4180 \mathrm{~cm}^{-1}$ can be attributed to the stretching-libration combination band. In addition, a broad feature at $974 \mathrm{~cm}^{-1}$ likely stems from the overtone of the previously established $558 \mathrm{~cm}^{-1}$ libration band. Under the assumption that the observable sub-band features can be correlated among the stretching fundamental, overtone, and combination band formations, the stretching-libration coupling $x_{\mathrm{s}, 1}^{\mathrm{Dim}}$ is found to be about $+43 \mathrm{~cm}^{-1}$ (see Table 2.3). Qualitatively, this distinctly positive value falls in line with expectations: a librational deflection of the donor $\mathrm{OH}$ group will tend to weaken the hydrogen bond, thus removing some of the red-shifting effect on the stretching vibration.

Furthermore, the diagonal $\mathrm{OH}$ stretching and libration anharmonicity constants $x_{\mathrm{s}, \mathrm{s}}^{\mathrm{Dim}}$ and $x_{1,1}^{\mathrm{Dim}}$ can be calculated from the assigned transitions. Here, the stretching constant of about $-97 \mathrm{~cm}^{-1}$ is reassuringly close to the jetFTIR value of $-99 \mathrm{~cm}^{-1}$ (see Section 2.2.1). All coupling constants show only little variation among the correlated sub-features, which indicates a highly regular behavior of the underlying vibrational dynamics across the different stretching/libration excitations.

While the matrix measurements readily enable the observation of the weak stretching-libration combination band, one still needs to bridge the gap to the desired, yet unattainable gas-phase situation. The previous monomer characterization by Perchard et al. ${ }^{114,121}$ is helpful in this regard as well, demonstrating that the host-guest interactions in neon matrices are small and systematic enough for the band positions to fall within few $\mathrm{cm}^{-1}$ of the gas phase. Naturally, this high robustness in the wavenumbers also carries over to the derived anharmonicity constants. Furthermore, an apparent breakdown of the $\mathrm{A} \leftarrow \mathrm{A}$ and $\mathrm{E} \leftarrow \mathrm{E}$ sub-state selection rules in the neon matrix $^{114}$ lends intensity to $\mathrm{A} \leftrightarrow \mathrm{E}$ type transitions, which allows to extract a 
Table 2.3: Sub-bands and anharmonicity constants (in $\mathrm{cm}^{-1}$ ) from matrixFTIR spectra (see Fig. 2.7) for donor $\mathrm{OH}$ stretching ("s") and libration ("l") motions, yielding diagonal anharmonicity constants $x_{\mathrm{s}, \mathrm{s}}$ and $x_{1,1}$, resp.; and stretching-libration combination sub-bands ("s+l"), yielding their mutual coupling $x_{\mathrm{s}, 1}$.

\begin{tabular}{rrrrrrrr}
\hline$\tilde{v}_{\mathrm{s}}$ & $(2 \tilde{v})_{\mathrm{s}}$ & $x_{\mathrm{s}, \mathrm{s}}$ & $\tilde{v}_{\mathrm{l}}$ & $(2 \tilde{v})_{1}$ & $x_{1,1}$ & $\tilde{v}_{\mathrm{s}+1}$ & $x_{\mathrm{s}, 1}$ \\
\hline 3568 & 6943 & -97 & $558^{\mathrm{a}}$ & 974 & -71 & 4168 & +42 \\
3560 & 6925 & -98 & & & & 4162 & +44 \\
3556 & 6921 & -96 & & & & 4157 & +43 \\
\hline
\end{tabular}

${ }^{\mathrm{a}}$ Ref. 103.

more comprehensive state-splitting picture from these experiments. Using the $\mathrm{E} \leftarrow \mathrm{A}$ transition of the stretching-torsion combination band, a monomer coupling constant of $x_{\mathrm{s}, \mathrm{t}}^{\mathrm{Mon}}$ of $+12.8 \mathrm{~cm}^{-1}$ was found, ${ }^{121}$ close to the corresponding gas-phase value of $+10.9 \mathrm{~cm}^{-1}$. This also holds true for the diagonal monomer $\mathrm{OH}$ stretching anharmonicity with an $\mathrm{A} \leftarrow \mathrm{A}$ neon-matrix value of $x_{\mathrm{s}, \mathrm{s}}^{\mathrm{Mon}}=-86.2 \mathrm{~cm}^{-1},{ }^{121}$ again close to the corresponding gas-phase values of -86.0 (see Table 2.1).

Concerning the dimer, the diagonal donor $\mathrm{OH}$ stretching anharmonicity of $x_{\mathrm{s}, \mathrm{s}}^{\mathrm{Dim}}=-97(1) \mathrm{cm}^{-1}$, averaged over the assigned sub-bands, agrees well with the jet-cooled gas-phase value of $-99 \mathrm{~cm}^{-1}$. Assuming similar agreement to uphold for anharmonicity constants that involve the libration, the data assembled in Table 2.3 should provide a viable benchmark for theoretical predictions. Based on the assumption that the anharmonicity constants from the neon-matrix experiments uphold to a good degree in the gas phase, the $\mathrm{OH}$ stretching and libration fundamental wavenumbers of 3575 and $560(10) \mathrm{cm}^{-1}$ from jet-FTIR experiments, ${ }^{14,103}$ together with the $x_{\mathrm{s}, 1}^{\mathrm{Dim}}=+43(5)$ coupling determined above, thus suggest a combination band position of $4178(15) \mathrm{cm}^{-1}$ in the gas phase.

From the matrix spectra shown in Figure 2.7, the stretching overtone and stretching-libration combination band patterns yield integrated absorbances of $0.50(10)$ and $0.20(3) \mathrm{cm}^{-1}$, respectively, resulting in a combination:overtone ratio of about $0.4(1)$. The fundamental band from these

\footnotetext{
${ }^{\ddagger}$ Unfortunately, Perchard et al. ${ }^{114,121}$ do not make their assigned sub-level transitions explicit, and state slightly different coupling constants as calculated herein from their assigned transitions. A detailed discussion of their data can be found in Ref. 17.
} 
spectra does not lend itself to a similar analysis due to the aforementioned optical saturation, but the jet experiments suggest a stretching fundamental:overtone ratio of 320(90) (see Section 2.2.1 and Reference 14) so that in all, the stretching-libration combination band can be estimated to be three orders of magnitude weaker than the fundamental. Of course, this direct comparison to gas-phase intensities is only meaningful if the polarizable matrix environment does not introduce substantial perturbations in the IR absorption coefficients of the analyte.

Previous matrix-FTIR experiments on the stretching overtone band suggest that the fundamental:overtone intensity ratios for the monomer indeed shows distinct variations across different matrix host materials, while the situation for the donor $\mathrm{OH}$ stretching vibration is well-comparable to the jet results. ${ }^{14}$ The librational mode, with its large-amplitude motion perpendicular to the hydrogen bond, might possibly experience a larger long-range polarizing interaction with the matrix environment, but this remains speculative without a direct observation of the stretching-libration band in a filetjet experiment. However, this endeavor is hampered by the low intensity of the combination band and its position close to the margins of the available optical filters.

\subsection{Quantum Chemical Calculations}

As per Equation (2.4), the starting point for an analysis of the observable dimerization red shift is the harmonic component, to which anharmonic corrections are added. One lasting difficulty in this scheme is that neither the harmonic shift nor the full off-diagonal sums are known from the experiment, seeing as this would require a full characterization of all fundamental bands and their combinations with the $\mathrm{OH}$ stretching vibration for both the monomer and the dimer. Some of the remaining terms must therefore be left to theoretical predictions, the most obvious one being the harmonic dimerization shift. However, most popular quantum chemical methods were found to be unreliable for calculating this quantity. ${ }^{15}$ An in-depth discussion of this effect is thus indispensable for the red-shift analysis.

On the anharmonic side, perturbational VPT2 predictions are currently the only feasible approach to the full 30-dimensional problem in the methanol dimer. This method will be discussed further below in its role of predicting both experimentally known diagonal anharmonicity constants and the remaining elusive off-diagonal sums. 


\section{Local and Canonical Results}

One obvious way to tackle the harmonic part of Equation (2.4) are quantum chemical calculations for which harmonic vibrational treatments are readily available and often still feasible. Standard MP2 and DFT methods have previously been found to predict harmonic red shifts $-\Delta \omega_{\mathrm{S}}$ on the order of $150 \mathrm{~cm}^{-1}$ and more (see Table 2.4). ${ }^{13,15,123}$ Together with the experimentally determined change in diagonal anharmonicity, $-2 \Delta x_{\mathrm{s}, \mathrm{s}}=27 \mathrm{~cm}^{-1}$, and the (localized) dimerization red shift of $-\Delta \tilde{v}_{\mathrm{S}}=109 \mathrm{~cm}^{-1}$, this would imply drastic off-diagonal contributions $\frac{1}{2} \sum \Delta x_{\mathrm{s}, i}$ on the order of $70 \mathrm{~cm}^{-1}$-whereas VPT2 predictions, despite carrying their own uncertainties, estimate these contributions to be significantly smaller. ${ }^{17,103}$ Furthermore, since the corresponding quantities encode the changes in anharmonic couplings from the monomer to the dimer situation, such large corrections would suggest a drastic impact of the highly localized hydrogen bond on the vibrational dynamics across the entire molecular aggregate, instead of only a few sensitive vibrational modes. It thus appears that either the harmonic calculations, their anharmonic counterparts, or both must be at fault.

Given that the influence of diagonal anharmonicity on the donor $\mathrm{OH}$ stretching wavenumber has been determined experimentally, ${ }^{14}$ any error in the harmonic shift is particularly unsatisfying in that it must be compensated with a factor of 2 by the off-diagonal sum in Equation (2.4). The uncertainties associated with the latter may thus become exceedingly large when deduced from the combined theoretical and experimental data, and obtaining accurate harmonic estimates is a key point in disentangling the observable dimerization shift.

A $-121 \mathrm{~cm}^{-1}$ reference value for the harmonic red shift was recently obtained from explicitly correlated local Coupled Cluster calculations. ${ }^{15}$ The MP2 results in Table 2.4 are of particular interest in this regard, seeing as they still overshoot this reference by some $30 \mathrm{~cm}^{-1}$ even in comparable local/explicit correlation combinations. The error in the harmonic red shifts can be reduced by including higher-order perturbations further along the MP series, similar to an MP4 study by Bleiber and Sauer on the water dimer. ${ }^{124}$ Based on the irregular behavior of the absolute stretching wavenumbers and interaction energies however, this appears to be a consequence of error compensation rather than a more faithful description of the electronic structure.

Concerning the local Coupled Cluster results in Table 2.4, the importance of subjecting all intermolecular orbital pairs to the high-level correlation treatment ("int") becomes clear. Based on default MOLPRO distance cutoffs, orbital pairs on different molecules are usually treated at the MP2 level if the "int" directive is not specified. From the data in Table 2.4, inclusion of 
Table 2.4: Collection of harmonic predictions for the methanol dimer: dissociation energies $D_{0}$ and $D_{\mathrm{e}}$ in $\mathrm{kJ} \mathrm{mol}^{-1}$; and wavenumbers $\omega$ for $\mathrm{O}-\mathrm{H}$ stretching and torsion/libration modes (subscripts "s", "t" and "l", resp.) in the monomer and dimer, together with corresponding dimerization shifts, in $\mathrm{cm}^{-1}$.

\begin{tabular}{|c|c|c|c|c|c|c|c|}
\hline & $D_{0}\left(D_{\mathrm{e}}\right)$ & $\omega_{\mathrm{S}}^{\mathrm{Mon}}$ & $\omega_{\mathrm{s}}^{\mathrm{Dim}}$ & $-\Delta \omega_{\mathrm{s}}$ & $\omega_{\mathrm{t}}^{\mathrm{Mon}}$ & $\omega_{1}^{\operatorname{Dim}}$ & $\Delta \omega_{1 / \mathrm{t}}$ \\
\hline B3LYP-D3 / VTZ & $25.9(31.9)$ & 3829 & 3687 & 142 & 306 & 700 & 395 \\
\hline B2PLYP-D3/VTZ & $25.6(31.5)$ & 3858 & 3722 & 136 & 307 & 697 & 390 \\
\hline MP2/VTZ & $24.2(29.9)$ & 3882 & 3740 & 142 & 309 & 699 & 390 \\
\hline MP2/aVTZ & $20.6(25.8)$ & 3860 & 3695 & 165 & 290 & 690 & 400 \\
\hline $\mathrm{MP} 2 / \mathrm{sVTZ}^{\mathrm{a}}$ & $20.5(25.8)$ & 3861 & 3692 & 169 & 294 & 695 & 401 \\
\hline LMP2 / VTZ & $20.0(25.4)$ & 3876 & 3758 & 118 & 309 & 661 & 352 \\
\hline $\mathrm{LMP} 2 / \mathrm{a}^{\prime} \mathrm{VTZ}^{\mathrm{b}}$ & $17.4(22.4)$ & 3859 & 3712 & 146 & 293 & 658 & 364 \\
\hline LMP2/aVTZ & $17.7(22.6)$ & 3856 & 3710 & 147 & 293 & 660 & 366 \\
\hline $\mathrm{LMP}$ / sVTZ ${ }^{\mathrm{a}}$ & $17.6(22.5)$ & 3857 & 3709 & 148 & 293 & 660 & 367 \\
\hline $\mathrm{LMP}_{\text {/ sVTZ }}{ }^{\mathrm{a}}$ & $17.3(22.6)$ & 3923 & 3805 & 118 & 296 & 657 & 360 \\
\hline $\mathrm{LMP} / \mathrm{sVTZ}^{\mathrm{a}}$ & $16.2(21.1)$ & 3823 & 3726 & 98 & 291 & 630 & 339 \\
\hline SCS-MP2/sVTZ ${ }^{\mathrm{a}}$ & $14.3(19.5)$ & 3858 & 3731 & 127 & 293 & 662 & 369 \\
\hline SCS-LMP2/sVTZ & $15.1(20.1)$ & 3852 & 3740 & 113 & 293 & 635 & 342 \\
\hline LMP2-F12 / VDZ-F12 & $19.0(24.0)$ & 3876 & 3714 & 162 & 298 & 675 & 377 \\
\hline $\mathrm{LCCSD}(\mathrm{T} 0) / \mathrm{sVTZ}^{\mathrm{a}}$ & $16.2(21.2)$ & 3829 & 3736 & 93 & 294 & 632 & 339 \\
\hline LCCSD(T0)(int)/sVTZ ${ }^{\mathrm{a}}$ & $16.5(22.0)$ & & 3723 & 106 & & 647 & 353 \\
\hline $\operatorname{LCCSD}(\mathrm{T} 0)($ int $) / \mathrm{a}^{\prime} \mathrm{VTZ}^{\mathrm{b}}$ & $16.3(21.8)$ & 3831 & 3727 & 104 & 293 & 644 & 351 \\
\hline LCCSD(T0*)-F12a/VDZ-F12 & $16.8(21.9)$ & 3862 & 3754 & 108 & 294 & 647 & 352 \\
\hline $\operatorname{LCCSD}\left(\mathrm{T} 0^{*}\right)-\mathrm{F} 12 \mathrm{a}(\mathrm{int}) / \mathrm{VDZ}-\mathrm{F} 12$ & $17.7(22.9)$ & & 3740 & 122 & & 660 & 365 \\
\hline LCCSD(T0*)-F12a/VTZ-F12 & $17.0(22.2)$ & 3863 & 3756 & 108 & 293 & 648 & 355 \\
\hline Best estimate $^{c}$ & $18.3(23.4)$ & & & 121 & & & \\
\hline
\end{tabular}

${ }^{a}$ Omitting diffuse functions on methyl hydrogens, see Sec. 1.3.3.

${ }^{\mathrm{b}}$ Omitting diffuse function on all hydrogens.

c From Ref. 15; see the text for details. 
intermolecular pairs at the Coupled Cluster level has a notable impact on the strength of the intermolecular interaction both in terms of its overall energy and the impact on the harmonic stretching wavenumbers. ${ }^{\S}$

Adding explicit correlation to the picture produces results that are close to the CBS limit already at the double-zeta level. Furthermore, since explicit correlation leaves the CBS limit of a method itself unchanged (see Section 1.3.1), the conventional LCCSD(T0) results demonstrate that triplezeta basis sets are still somewhat too small for spectroscopic predictions. Interestingly, the effects of including intermolecular pairs at Coupled Cluster level ("int") are quite similar among the LCCSD(T0) and LCCSD(T0*)-F12a results. Even at the explicitly correlated VTZ-F12 level (not included in Table 2.4), a comparable $1.0 \mathrm{~kJ} \mathrm{~mol}^{-1}$ increase in the electronic dissociation energy $D_{\mathrm{e}}$ was found. It thus appears that the "int" effects are approximately additive and rather insensitive to CBS limit convergence. A set of bestestimate values was thus obtained by conducting a structure optimization and frequency calculation at the VTZ-F12 (non-"int") level, re-calculating the interaction energy at the VQZ-F12 (non- "int") level, and adding the VDZ-F12 "int" effects in the wavenumbers and energies. ${ }^{15}$ This composite approach yields a best-estimate interaction energy of $D_{0}=18.3 \mathrm{~kJ} \mathrm{~mol}^{-1}$ and the aforementioned harmonic dimerization shift of $121 \mathrm{~cm}^{-1}$. With respect to these composite results, the LCCSD(T0*)-F12a(int)/VDZ-F12 predictions already provide a remarkable accuracy at reasonable computational costs. To allow for remaining basis set incompleteness effects and impacts from the various underlying approximations, a $\pm 5 \mathrm{~cm}^{-1}$ error bar is assumed for the harmonic dimerization shift.

A comparison of the MP2, LMP2 and LCCSD(T0) data presented in Table 2.4 further provides a consistency check for the partially and selectively augmented a'VTZ and sVTZ basis set constructions introduced in Section 1.3.3. For the methanol dimer, diffuse functions in sVTZ were added on the donor and acceptor $\mathrm{OH}$ protons, leaving the hydrogen atoms of the methyl groups with the pure VTZ kernel. The results indicate that the construction is quite robust in all cases, which is plausible in that the methyl groups do not partake to a significant degree in the association of the two methanol sub-units. Meanwhile, augmenting only the $\mathrm{OH}$ protons reduces the number of basis functions from 368 (aVTZ) to 314 (sVTZ), and while practical limitations have rendered it impractical to conduct a full LCCSD(T0)(int)/aVTZ treatment of the methanol dimer within the course

\footnotetext{
${ }^{\S}$ Conversely, the default LMP2 method in MOLPRO keeps all correlated orbital pairs at the MP2 level without reducing the computational rigor for more distant ones. The "int" directive therefore only takes effect in the local Coupled Cluster calculations presented herein, and is obviously irrelevant in the monomer context.
} 
of this thesis, the corresponding sVTZ calculations were still within reach. If the validity of a selective augmentation scheme can be validated, its use is thus favorable over the fully augmented basis set. Further omitting diffuse functions on all hydrogen atoms indiscriminately leads to the a'VTZ basis set. $^{99}$ Here, the LMP2 and LCCSD(T0) results in Table 2.4 do not differ drastically from their sVTZ (and, presumably, also the full aVTZ) counterparts. Further stripping the diffuse functions also from all heavy atoms and retaining only the core VTZ basis set has a significant impact on all properties at the (L)MP2 level. This is of interest in the context of the VPT2 calculation presented further below, since they were carried out exclusively in the VTZ basis set for stability reasons. Thus, it is generally important to include diffuse functions at least on heavy atoms in order to obtain adequate results. Still, the additional cost of the sVTZ construction over a'VTZ is small, and its practicability makes it a viable candidate for further use.

Apart from the $\mathrm{OH}$ dimerization red shift and the associated intensity modulations, one can also assume the blue-shifting effect of the $\mathrm{OH} \cdots \mathrm{O}$ contact on the $\mathrm{OH}$ torsion motion to constitute another measure for the interaction strength. Listed in Table 2.4 are the harmonic wavenumbers of the free monomer $\mathrm{OH}$ torsion and its librational counterpart in the dimer. Across the chosen methods, the monomer values fall in a range of less than $20 \mathrm{~cm}^{-1}$, with more accurate treatments agreeing on a value around $293 \mathrm{~cm}^{-1}$. The variations in the dimer libration wavenumbers are somewhat larger, but still center around a high-level $660 \mathrm{~cm}^{-1}$ result with deviations of up to $40 \mathrm{~cm}^{-1}$. The dimer-monomer shift of 350 to $400 \mathrm{~cm}^{-1}$ indicates that the shallow torsional potential in the monomer adopts a much more confined character due to the attractive and directing influence of the acceptor center.

The mediocre performance of the MP2 calculations for the harmonic red shift leaves room for improvement. Based on a suggestion by Grimme, ${ }^{125}$ the contributions of parallel and antiparallel spin pairs to the MP2 correlation energy can be weighted with individual factors, which is termed "SpinComponent Scaling" (SCS). The two contained parameters were originally determined by Grimme from fitting the resulting correlation energies to a benchmark set. A stringent theoretical reasoning for the individual scaling of the different spin-pair contributions has been provided by Fink. ${ }^{126}$ In practical terms, SCS-MP2 has been found to improve the dissociation energies of dispersion-dominated intermolecular interactions, but in turn underestimate classical hydrogen bonds. ${ }^{127,128}$

In the case of methanol, SCS-LMP2 predicts reasonable monomer and dimer stretching wavenumbers with respect to the best Coupled Cluster estimates, whereas LMP2 only agrees on the monomer value. This leads to an SCS-LMP2 harmonic dimerization shift of $113 \mathrm{~cm}^{-1}$ for the methanol dimer, 
much closer to the best available estimate $\left(121 \mathrm{~cm}^{-1}\right)$ than the pure LMP2 value $\left(148 \mathrm{~cm}^{-1}\right)$. While dissociation energies from conventional, unscaled MP2 approaches are still favorable, the harmonic spectroscopic predictions appear to profit from applying SCS in this case. However, it remains unclear at this point whether these superficially improved results indeed reflect a more faithful description of the electronic structure in the methanol dimer, or rather some fortuitous error compensation. The viability of the approximation will be further tested on other systems throughout this work.

\section{LMOMO Dissection of Dimerization Shift}

Within the framework of local electron correlation, the LMOMO method provides yet another option to reduce the computational demand while allowing to approach a desired accuracy. To this end, user-defined subsets of atoms in a molecular system are used to group the localized domains into "regions" which are to be treated at a certain computational level, while the remainder of the system is demoted to some less expensive method. Such an LCCSD(T0):LMP2/aVTZ approach was undertaken for the methanol dimer, with instructive results reflecting on the notorious MP2 overestimation of the harmonic dimerization shift. ${ }^{15}$ An more detailed look at the region definitions and corresponding MOLPRO inputs is provided in Appendix C.

From a standard LMP2/aVTZ starting point (step (a) in Figure 2.8), treating the electron pair in the donor $\mathrm{O}-\mathrm{H}$ bond at the Coupled Cluster level (b) already reduces the harmonic red shift by almost $40 \mathrm{~cm}^{-1}$. The impact of extending the high-level region to the acceptor $\mathrm{O}-\mathrm{H}$ bond and all oxygen lone pairs (c, d) is significantly smaller, and further promoting the intermolecular excitations to the CC level (e) even tends to widen the monomer-dimer gap again. The most important difference between full LMP2 and LCCSD(T0) treatments thus appears to be the inclusion of singles excitations in the donor $\mathrm{O}-\mathrm{H}$ bond, which MP2 naturally neglects. Furthermore, the LMP2-F12/VDZ-F12 results in Table 2.4 suggest that the method still significantly exaggerates the harmonic dimerization shift when brought closer to the basis set limit. An application of the LMOMO method with explicitly correlated methods is still pending, but will be highly desirable also in the context of larger molecular systems.

The physical justification of the LMOMO scheme itself is again given by the local nature of electron correlation, suggesting that the interaction characteristics of the two $\mathrm{OH}$ groups are mostly insensitive to the treatment of the largely non-participating moieties. From this standpoint, steps (d) and (e) in Figure 2.8 can be compared to the full LCCSD(T0)/sVTZ and LCCSD(T0)(int)/sVTZ results; the relevant data are assembled in Table 2.5. 


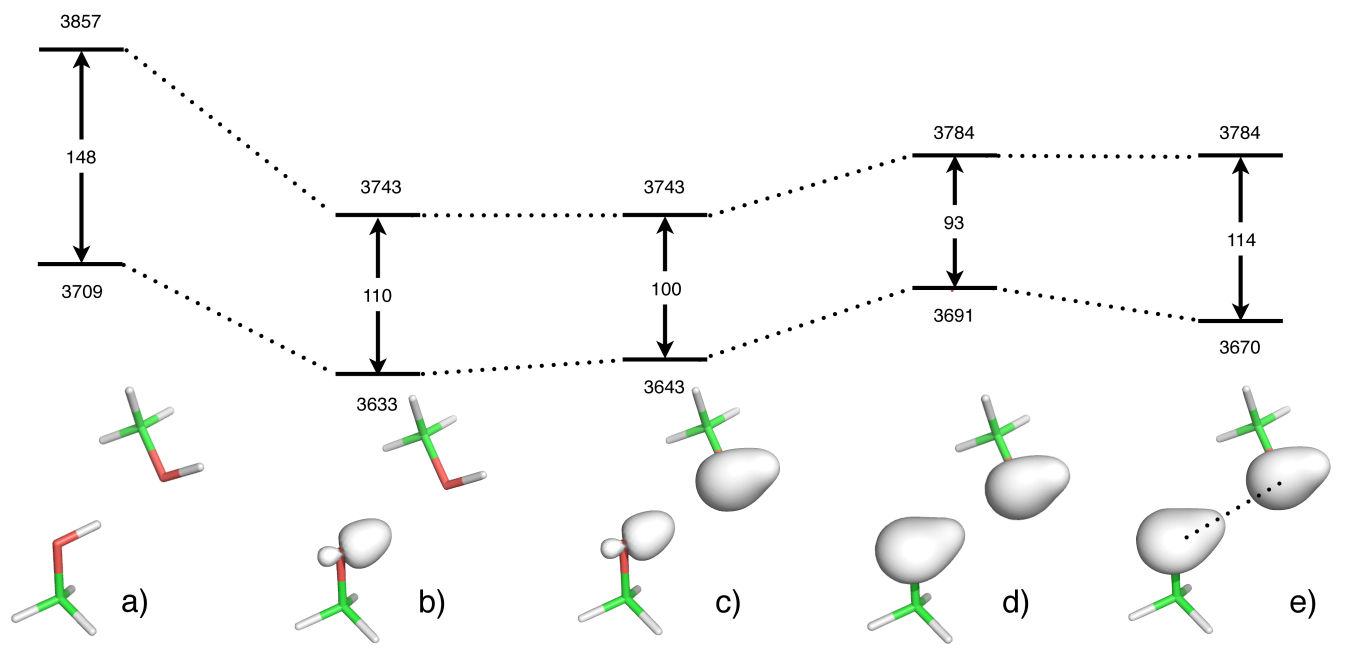

Figure 2.8: Visualization of LMOMO calculations for the methanol dimer (courtesy of R. A. Mata, reprinted from Ref. 16 with permission of AIP Publishing). Displayed are density plots of the orbitals which are successively included in the LCCSD(T0) region, together with an energy level scheme and harmonic wavenumbers (in $\mathrm{cm}^{-1}$ ) for the monomer and dimer donor $\mathrm{OH}$ stretching modes.

Table 2.5: Comparison of LMOMO and LCCSD(T0) results using the sVTZ basis set construction, with and without inclusion of intermolecular pairs ("int") and the $\mathrm{C}-\mathrm{O}$ bonds ("+C-O") at the Coupled Cluster level. Given are the harmonic monomer and dimer donor $\mathrm{OH}$ stretching wavenumbers $\left(\omega_{\mathrm{s}}\right.$, in $\left.\mathrm{cm}^{-1}\right)$, together with the dimerization shifts and electronic/zero-point corrected dissociation energies $\left(D_{0}\right.$ and $D_{\mathrm{e}}$, in $\left.\mathrm{kJ} \mathrm{mol}^{-1}\right)$.

\begin{tabular}{llrrr}
\hline & & $\mathrm{LMOMO}^{\mathrm{a}}$ & $\mathrm{LMOMO} \mathrm{C}-\mathrm{O}$ & $\mathrm{LCCSD}(\mathrm{T} 0)$ \\
\hline non-"int” & $\omega_{\mathrm{s}}^{\text {Mon }}$ & 3784 & 3836 & 3829 \\
& $\omega_{\mathrm{s}}^{\text {Dim }}$ & 3691 & 3743 & 3736 \\
& $-\Delta \omega_{\mathrm{s}}$ & 93 & 93 & 93 \\
& $D_{0}\left(D_{\mathrm{e}}\right)$ & $16.1(21.0)$ & $16.1(21.2)$ & $16.2(21.2)$ \\
“int” & $\omega_{\mathrm{s}}^{\text {Mon }}$ & 3784 & 3836 & 3829 \\
& $\omega_{\mathrm{s}}^{\text {Dim }}$ & 3670 & 3730 & 3723 \\
& $-\Delta \omega_{\mathrm{s}}$ & 114 & 106 & 106 \\
& $D_{0}\left(D_{\mathrm{e}}\right)$ & $17.0(22.1)$ & $17.0(22.1)$ & $16.5(22.0)$ \\
\hline
\end{tabular}

a Calculations (d), (e) in Fig. 2.8. 
Partitioning the methanol molecules in the scheme discussed above has a significant impact on the absolute harmonic wavenumbers, red-shifting them by some 40 to $50 \mathrm{~cm}^{-1}$, whereas the resulting dimerization shifts and dissociation energies still compare well among the calculations. The discrepancies in the absolute wavenumbers can be significantly reduced by further including the $\mathrm{C}-\mathrm{O}$ bonds at the Coupled Cluster level ("LMOMO+C-O" in Table 2.5), providing results within $10 \mathrm{~cm}^{-1}$ of the full LCCSD(T0)(int) calculations.

\subsubsection{Anharmonic Calculations}

While establishing a high-level harmonic reference for the dimerization shift allows to deduce estimates for the off-diagonal sum in Equation (2.4), it does not provide detailed insight into its individual components. To this end, the complete anharmonic coupling matrices in the methanol monomer and dimer were obtained from a number of VPT2 calculations at the MP2 and B3LYP levels of theory ${ }^{14,103}$ and shall be discussed in detail below. In addition, potential energy curves for the $\mathrm{OH}$ stretching vibrations were calculated at the $\operatorname{LCCSD}\left(\mathrm{T}^{*}\right.$ )-F12a(int) level and subjected to numerical solutions of the vibrational Schrödinger equation in order to obtain diagonally anharmonic estimates for this level of theory.

\section{VPT2 Calculations}

As outlined in Section 1.3.3, the non-augmented VTZ basis set was used for all calculations in order to improve the numerical stability of the VPT2 results, and all DFT calculations furthermore only employed zero-damping. Still, there is a distinct impact of the DFT instabilities that are outlined in Section 1.3.3 and further discussed in Reference 17.

First, the DFT VPT2 calculations are quite sensitive to the underlying molecular structure, which causes deviations in the anharmonic predictions already for different input structures to the preceding geometry optimizations. In the case of the methanol monomer, this effect alone leads to the stretching-torsion coupling, $x_{\mathrm{s}, \mathrm{t}}^{\mathrm{Mon}}$, being predicted at the B3LYP-D3/VTZ level to be between +3 and $+8 \mathrm{~cm}^{-1}$ with a drastic outlier of $-20 \mathrm{~cm}^{-1}$. In the dimer, the second-lowest normal mode-corresponding to the hindered torsion of the entire donor methanol molecule around its pseudo-figure axisshows an exaggerated sensitivity to numerical instabilities, with unphysical, often imaginary wavenumbers in many calculations. Conversely, MP2 behaves better in this regard and only experiences inconsistencies due to the 
Table 2.6: VPT2-anharmonic results (in $\mathrm{cm}^{-1}$ ) for the methanol monomer and dimer donor on different levels of theory (see also Refs. 16,17): diagonal $\mathrm{OH}$ stretching anharmonicity constants $\left(x_{\mathrm{s}, \mathrm{s}}\right)$; stretching-torsion and libration coupling constants $\left(x_{\mathrm{s}, \mathrm{t}}\right.$ and $\left.x_{\mathrm{s}, \mathrm{l}}\right)$; and remaining primed-sum terms as per Eqs. (2.5) and (2.6). Also given are the available experimental data, using the localized monomer $x_{\mathrm{s}, \mathrm{t}}$ reference from Sec. 2.1.

\begin{tabular}{|c|c|c|c|c|c|c|}
\hline & \multicolumn{3}{|l|}{ Mon } & \multicolumn{3}{|l|}{ Dim } \\
\hline & $x_{\mathrm{s}, \mathrm{s}}$ & $x_{\mathrm{s}, \mathrm{t}}$ & $\sum^{\prime} x_{\mathrm{s}, j}$ & $x_{\mathrm{s}, \mathrm{s}}$ & $x_{\mathrm{s}, 1}$ & $\sum^{\prime} x_{\mathrm{s}, j}$ \\
\hline B2PLYP-D3/VTZ & -86 & +4 & -28 & -102 & +58 & +15 \\
\hline B3LYP-D3/VTZ & -87 & +3 & -26 & -104 & +58 & +25 \\
\hline MP2/VTZ & -83 & +9 & -30 & -102 & +59 & +9 \\
\hline exp. & $-86(1)$ & $+15(6)$ & & -99 & $+43(5)$ & \\
\hline
\end{tabular}

apparent accuracy issues in the storage and retrieval of molecular structures as outlined in Section 1.3.3.

The results assembled in Table 2.6 show that the diagonal $\mathrm{OH}$ stretching anharmonicity constant $x_{\mathrm{s}, \mathrm{s}}^{\mathrm{Dim}}$ is reproduced with a slight, but systematic overestimation among the chosen methods. Any disagreement between theory and experiment in this value is exacerbated by the pre-factor of 2 in Equation (2.4), and the resulting deviations of up to $+10 \mathrm{~cm}^{-1}$ in the dimer-monomer correction $-2 \Delta x_{\mathrm{s}, \mathrm{s}}$ amount to roughly one third of the true $-27 \mathrm{~cm}^{-1}$ experimental value. The stretching-libration couplings are similarly misjudged in magnitude, exceeding the neon-matrix experiment by some $15 \mathrm{~cm}^{-1}$, again about one third of the experimental value. Fortuitously, the two errors tend to cancel in their overall effect due to the opposite signs.

For the primed-sum terms $\Sigma^{\prime} x_{\mathrm{s}, j}$ according to Equation (2.5), the results suggest an average value of about $+16(9) \mathrm{cm}^{-1}$ in the dimer, and a more robust value of $-28(2) \mathrm{cm}^{-1}$ in the monomer, with a dimer-monomer difference of $\Sigma^{\prime} \Delta x_{\mathrm{s}, j}=+44(10) \mathrm{cm}^{-1}$. The sizable uncertainties in these numbers stem mostly from the numerical instabilities. Conversely, both the diagonal stretching anharmonicity $x_{\mathrm{s}, \mathrm{s}}^{\mathrm{Dim}}$ and, somewhat surprisingly, also the stretching-libration coupling $x_{\mathrm{s}, 1}^{\mathrm{Dim}}$ in the dimer are robust to within about $2 \mathrm{~cm}^{-1}$. Since these two coupling constants are the most important anharmonic contributions in the methanol dimer, ${ }^{17}$ their stability among the calculations is reassuring for their direct use in the combined experimental and theoretical discussion. 
Table 2.7: Absolute band positions and related anharmonicity constants (in $\mathrm{cm}^{-1}$ ) for the methanol monomer (top) and dimer donor (bottom) from VPT2 calculations at the three chosen levels of theory, all using the

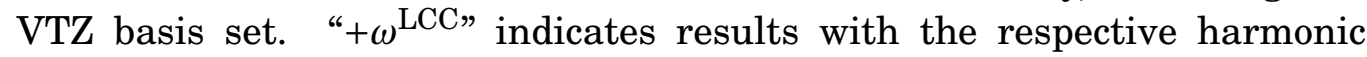
wavenumbers replaced by the LCCSD(T0*)-F12a(int)/VDZ-F12 values (see also Refs. 15,103). Also given are experimental results ("exp."), see the text for details.

\begin{tabular}{|c|c|c|c|c|c|c|c|c|}
\hline & $\tilde{v}_{\mathrm{s}}$ & $(2 \tilde{v})_{\mathrm{S}}$ & $x_{\mathrm{s}, \mathrm{s}}$ & $\tilde{v}_{1 / \mathrm{t}}$ & $(2 \tilde{v})_{1 / t}$ & $x_{1,1 / t, \mathrm{t}}$ & $\tilde{v}_{\mathrm{s}+1 / \mathrm{t}}$ & $x_{\mathrm{s}, 1 / \mathrm{t}}$ \\
\hline \multicolumn{9}{|c|}{ Monomer } \\
\hline B2PLYP-D3 & 3674 & 7175 & -86 & 241 & 413 & -35 & 3919 & +4 \\
\hline$+\omega^{\mathrm{LCC}}$ & 3678 & 7183 & & 229 & 388 & & 3911 & \\
\hline B3LYP-D3 & 3645 & 7117 & -87 & 233 & 391 & -37 & 3881 & +3 \\
\hline$+\omega^{\mathrm{LCC}}$ & 3678 & 7182 & & 221 & 368 & & 3902 & \\
\hline \multirow[t]{2}{*}{ MP2 } & 3706 & 7247 & -83 & 257 & 453 & -30 & 3972 & +9 \\
\hline & 3686 & 7206 & & 243 & 425 & & 3937 & \\
\hline \multirow[t]{2}{*}{ exp. } & 3684 & 7196 & $-86(1)$ & $230(20)$ & & & $3930(30)$ & $+15(6)$ \\
\hline & & & & Dimer & & & & \\
\hline B2PLYP-D3 & 3554 & 6904 & -102 & 589 & 1088 & -43 & 4200 & +58 \\
\hline$+\omega^{\mathrm{LCC}}$ & 3572 & 6939 & & 551 & 1016 & & 4181 & \\
\hline B3LYP-D3 & 3520 & 6831 & -104 & 586 & 1079 & -45 & 4164 & +58 \\
\hline$+\omega^{\mathrm{LCC}}$ & 3573 & 6937 & & 545 & 1000 & & 4176 & \\
\hline \multirow[t]{3}{*}{ MP2 } & 3571 & 6939 & -102 & 592 & 1088 & -46 & 4222 & +59 \\
\hline & 3571 & 6939 & & 552 & 1012 & & 4183 & \\
\hline & 3575 & 6951 & $-99(1)$ & 558 & 974 & $-71(5)$ & $4178(5)$ & $+43(5)$ \\
\hline
\end{tabular}

In addition to the anharmonicity constants listed in Table 2.6 , it is instructive to judge the intrinsic performance of the VPT2 calculations in predicting the overall wavenumbers of some relevant transitions. Table 2.7 assembles predictions at the three chosen levels of theory for the fundamental, overtone and combination bands in the methanol monomer and dimer. Experimental data are included to provide a reference for the calculations, with an estimated gas-phase value for the stretching-libration combination wavenumber $\tilde{v}_{\mathrm{s}+1}^{\text {Dim }}$ in the dimer based on the fundamental transitions $\tilde{v}_{\mathrm{s}}^{\text {Dim }}$ and $\tilde{v}_{1}^{\text {Dim }}$, and the $x_{\mathrm{s}, 1}^{\mathrm{Dim}}$ coupling element. Concerning the monomer, the ambiguities in the localization approaches discussed in Section 2.1 are too large for the torsional motion to serve as a stringent benchmark for the calculations, for which reason its experimental overtone wavenumber and diagonal anharmonicity constant are omitted in Table 2.7. 
In the dimer, the two chosen DFT levels tend to underestimate the $\mathrm{OH}$ stretching fundamental wavenumber $\tilde{v}_{\mathrm{S}}^{\text {Dim }}$ in comparison to the experiment, while MP2 comes close to it. The harmonic results in Table 2.4 suggest that the DFT errors might be mostly contained in the harmonic wavenumbers, which are also too low in comparison to the high-level Coupled Cluster results, whereas MP2/VTZ coincidentally provides a harmonic stretching wavenumber close to the high-level values. Through the general additivity of all harmonic and anharmonic effects in Equation (1.1), one can substitute all harmonic wavenumbers with the respective highlevel LCCSD(T0*)-F12a(int)/VDZ-F12 results before including the anharmonic corrections. These harmonic "updates" are indicated in Table 2.7 by " $+\omega^{\mathrm{LCC}}$. For the dimer donor, using the $3740 \mathrm{~cm}^{-1}$ reference wavenumber then leads to a good agreement of all calculated band positions with the jet-FTIR experiment. Similar improvements are seen in the first overtone $(2 \tilde{v})_{\mathrm{s}}^{\mathrm{Dim}}$, although the remaining gap to the experiment is larger than for the fundamental. This can likely be explained by the slightly overestimated diagonal anharmonicity constants $x_{\mathrm{s}, \mathrm{s}}^{\mathrm{Dim}}$, again due to their larger pre-factors in the vibrational term formulas.

Concerning the dimer libration band with a high-level harmonic reference of $\omega_{\mathrm{s}}^{\mathrm{Dim}}=660 \mathrm{~cm}^{-1},{ }^{103}$ the agreement is again satisfactory for the fundamental, but systematically worse for the overtone due to the underestimated anharmonicity constant $x_{1,1}^{\mathrm{Dim}}$ and its consequentially insufficient red-shifting contribution. However, since both the stretching and libration fundamental wavenumbers are in good agreement with the experiment at least after the harmonic updates, the combination band is similarly well reproduced.

For the monomer, the $\mathrm{OH}$ stretching mode again exhibits a beneficial effect of including the LCCSD(T0*)-F12a/VDZ-F12 harmonic wavenumber of $3862 \mathrm{~cm}^{-1}$. As mentioned before, the ambiguities in the torsional localization obfuscate the corresponding results, and no clear advantage of the high-level harmonic updates is apparent. In addition, it shall be stressed here again that the localization approaches lack a direct physical meaning and are only included to compare the experimental and theoretical results.

As indicated in Table 2.8, the VPT2 calculations perform adequately in modeling the 320(90)-fold decrease of the stretching overtone intensity in relation to the fundamental. However, the matrix-FTIR spectra indicate the stretching-libration combination band to be weaker by a factor of about 0.4 than the stretching overtone, whereas the VPT2 calculations predict it to be three times stronger by an average ratio of 3.4(5). Although matrix effects as discussed in Section 2.2.2 cannot be excluded to play a role in this intensity reversal, it is likely that most of these errors are contained in the VPT2 calculations, seeing as they also misjudge the diagonal libration 
Table 2.8: Intensity ratios of stretching overtone to fundamental bands

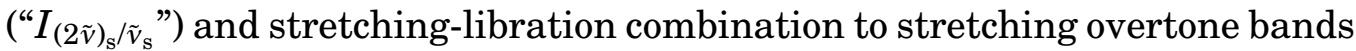
(“ $I_{\tilde{v}_{\mathrm{s}+1} /(2 \tilde{v})_{\mathrm{s}}}$ ”) from VPT2 calculations and jet/matrix experiments.

\begin{tabular}{lll}
\hline & $I_{(2 \tilde{v})_{\mathrm{s}} / \tilde{v}_{\mathrm{s}}}$ & $I_{\tilde{v}_{\mathrm{s}+1} /(2 \tilde{v})_{\mathrm{s}}}$ \\
\hline B2PLYP-D3 & 338 & 3.6 \\
B3LYP-D3 & 296 & 2.8 \\
MP2 & 430 & 3.9 \\
exp. & $320(90)$ & $0.4(1)$ \\
\hline
\end{tabular}

anharmonicity constant $x_{1,1}^{\mathrm{Dim}}$ and the stretching-libration coupling element $x_{\mathrm{s}, 1}^{\mathrm{Dim}}$.

Altogether, the results suggest that anharmonic VPT2 predictions provide adequate estimates for absolute $\mathrm{OH}$ stretching and libration band positions at the chosen levels of theory, at least when relating them to high-level harmonic wavenumbers. However, a deeper analysis of the contributing terms reveals that the predictions profit from some systematic error compensation in the two most important anharmonicity constants: the diagonal stretching anharmonicity $x_{\mathrm{s}, \mathrm{s}}^{\mathrm{Dim}}$ and the stretching-libration coupling $x_{\mathrm{s}, 1}^{\mathrm{Dim}}$. The intensity ratio of the stretching fundamental and overtone transitions is estimated realistically, but comparability with the experiment again breaks down as soon as the libration is involved. One can thus surmise fundamental flaws of the perturbational approach with respect to the shallow potential of this mode, exacerbated in DFT calculations by numerical instabilities which introduce large uncertainties to the results.

\section{1-D Numerical Solutions}

Potential energy curves along the (donor) $\mathrm{OH}$ stretching normal coordinates in the methanol monomer and dimer were built using the LCCSD(T0*)F12a(int)/VDZ-F12 method in order to assess its predictive power for the diagonal anharmonicity content $x_{\mathrm{s}, \mathrm{s}}$ in these modes. ${ }^{16}$ The resulting energies were fitted with a modified Morse potential of the form

$$
V(Q)=C\left[1-\exp \left(-\sum_{i=1}^{5} b_{i} Q^{i}\right)\right]^{2} .
$$

This expression was found to provide an improved description of the potential energies over the standard Morse formula, which holds true even at 
large values of $Q$ where the acceptor atom introduces a short-range confining potential barrier on top of its long-range attractive influence. Numerical solutions to the 1-D vibrational Schrödinger equation were calculated by means of the MATLAB routines employed before in Section 2.1, using the reduced mass as obtained by the preceding normal-mode analysis. In comparison to the results in Reference 16, some modifications were made to the routines in order to enhance their numerical stability (see Appendix B for further details and potential fit parameters), but were found to have no noticeable impact on the results.

The fitted potentials yield harmonic monomer and dimer $\mathrm{OH}$ stretching wavenumbers of 3861.6 and $3737.2 \mathrm{~cm}^{-1}$, respectively, thus reproducing the usual normal-mode analyses given in Table 2.4 to within $1 \mathrm{~cm}^{-1}$ for the monomer and $3 \mathrm{~cm}^{-1}$ for the dimer. From the eigenstates calculated in these potentials, one further obtains monomer $\mathrm{OH}$ stretching fundamental and overtone wavenumbers of 3688.7 and $7206.7 \mathrm{~cm}^{-1}$, respectively, with a resulting diagonal anharmonicity constant of $x_{\mathrm{s}, \mathrm{s}}^{\mathrm{Mon}}=-85.4 \mathrm{~cm}^{-1}$; and dimer donor wavenumbers of 3546.8 and $6902.2 \mathrm{~cm}^{-1}$, yielding $x_{\mathrm{s}, \mathrm{s}}^{\mathrm{Dim}}=-95.7 \mathrm{~cm}^{-1}$. Assuming these results to be accurate, this implies mostly vanishing offdiagonal corrections in the monomer, and $\mathrm{a} \sim 30 \mathrm{~cm}^{-1}$ blue shift in the dimer band. In comparison, the VPT2 results in Table 2.6 predict off-diagonal anharmonic blue shifts (including the prefactor of 1/2) on the order of $10 \mathrm{~cm}^{-1}$ in the monomer and $30 \mathrm{~cm}^{-1}$ in the dimer when substituting the experimentally determined values for the stretching-torsion and stretching-libration couplings. Taking into account possible fitting inaccuracies in the 1-D potential curves, a probable shortcoming of the perturbational VPT2 approach at least for some of the involved vibrational couplings, and the difficult monomer localization of the torsional motion, the numerical solutions can be deemed to be compatible with the combined experimental and theoretical data presented above.

Unfortunately, the local Coupled Cluster methods implemented in the available MOLPRO version did not allow for the calculation of dipole moments, and intensity estimates were not available at this level of theory neither in a harmonic nor a 1-D anharmonic picture. Beyond the estimates determined above, a direct variational determination of the stretching-torsion/libration coupling constants from LCCSD(T0*)-F12a potential energy surfaces would be greatly desirable. 


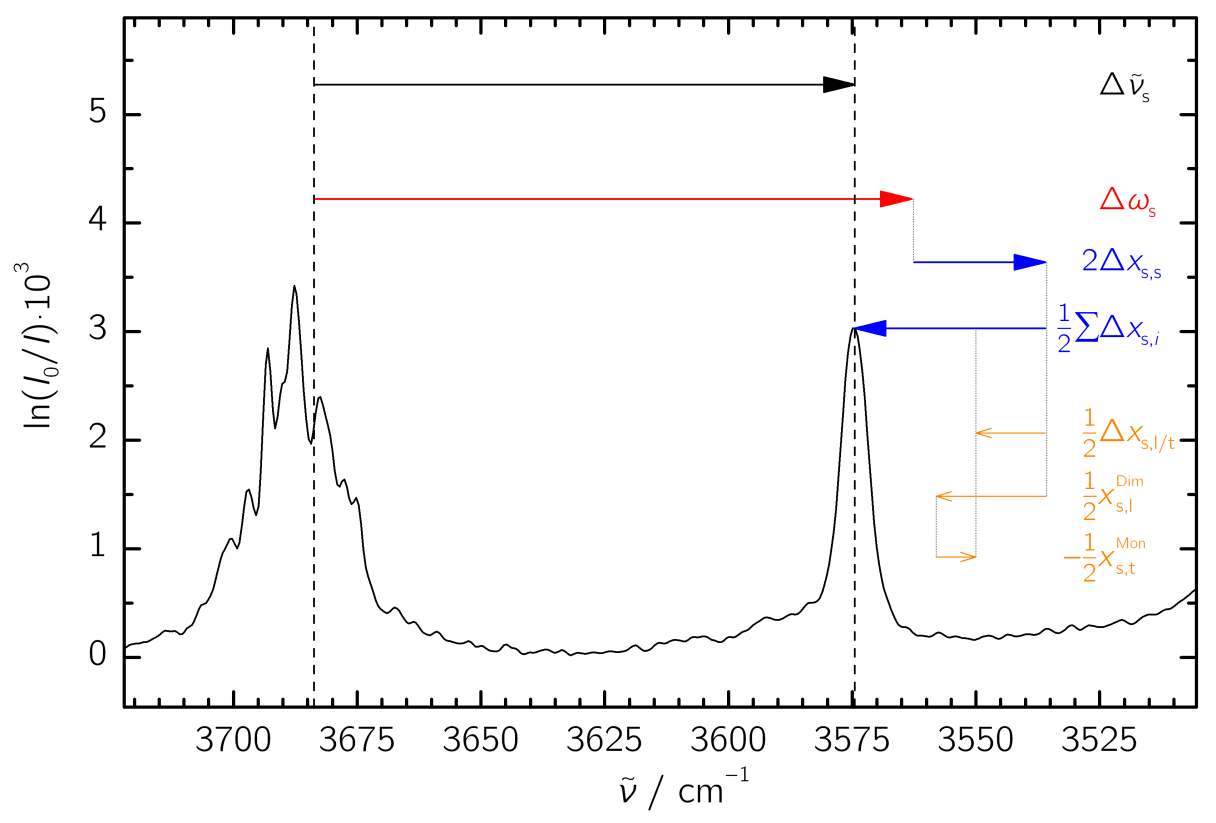

Figure 2.9: Visual dissection of the overall methanol $\mathrm{OH}$ dimerization red shift (black) into its harmonic (red) and anharmonic (blue) components, with the stretching-torsion/libration couplings (orange) separated from the latter.

\subsection{OH Dimerization Red Shift}

After collecting all available harmonic and anharmonic contributions pertaining to the monomer and dimer $\mathrm{OH}$ stretching bands (see Table 2.9), the data can be assembled in order to explain the overall observable dimerization shift,

$$
-\Delta \tilde{v}_{\mathrm{s}}=-\Delta \omega_{\mathrm{s}}-2 \Delta x_{\mathrm{s}, \mathrm{s}}-\frac{1}{2}\left[\Delta x_{\mathrm{s}, 1 / \mathrm{t}}+\sum^{\prime} \Delta x_{\mathrm{s}, j}\right] .
$$

A dissection of the dimerization shift into its individual components is visualized in Figure 2.9. With the stretching-libration coupling of $x_{\mathrm{s}, 1}^{\mathrm{Dim}}=$ $+43(5) \mathrm{cm}^{-1}$ in the dimer (Section 2.2.2) and the corresponding $x_{\mathrm{s}, \mathrm{t}}^{\mathrm{Mon}}=$ $+15(6) \mathrm{cm}^{-1}$ value for the monomer (Section 2.1), the difference term $\Delta x_{\mathrm{s}, 1 / \mathrm{t}}$ amounts to about $+28(8) \mathrm{cm}^{-1}$. Adding the best-estimate harmonic red shift of $-\Delta \omega_{\mathrm{S}}=121(5) \mathrm{cm}^{-1}$ (Section 2.3) and the diagonal anharmonic difference of $-2 \Delta x_{\mathrm{s}, \mathrm{s}}=+27(2) \mathrm{cm}^{-1}$ (Section 2.2.1), these data imply a primed sum of $\frac{1}{2} \sum^{\prime} \Delta x_{\mathrm{s}, j}=+25(7) \mathrm{cm}^{-1}$ in order to meet the overall observable $-\Delta \tilde{v}_{\mathrm{s}}=109(1) \mathrm{cm}^{-1}$ shift. 
Table 2.9: Summary of harmonic and anharmonic terms (in $\mathrm{cm}^{-1}$ ) in the localized methanol monomer and dimer donor, together with negative dimermonomer differences (“- $\Delta$ ”) in all quantities. The overall off-diagonal and primed-sum values are deduced from the preceding data, and the primed sums are compared to VPT2 predictions in the last line.

\begin{tabular}{|c|c|c|c|}
\hline & Mon & Dim & $-\Delta$ \\
\hline$\omega_{\mathrm{s}}$ & 3862 & 3740 & $+121^{\mathrm{a}}$ \\
\hline$\tilde{v}_{\mathrm{S}}$ & 3684 & 3575 & +109 \\
\hline $2 x_{\mathrm{s}, \mathrm{s}}$ & -171 & -198 & +27 \\
\hline$\Rightarrow \frac{1}{2} \sum x_{\mathrm{s}, i}$ & -7 & +33 & -40 \\
\hline$\frac{1}{2} x_{\mathrm{s}, 1 / \mathrm{t}}$ & +8 & +22 & -14 \\
\hline$\Rightarrow \frac{1}{2} \sum^{\prime} x_{\mathrm{s}, j}$ & -15 & +11 & -26 \\
\hline VPT2: & -14 & +8 & -22 \\
\hline
\end{tabular}

${ }^{\text {a }}$ Best-estimate value, see Sec. 2.3.

The VPT2 results, at $\frac{1}{2} \sum^{\prime} \Delta x_{\mathrm{s}, j}=+22(5) \mathrm{cm}^{-1}$, perform satisfactorily in predicting the primed-sum quantity, but it is unclear to what extent this may be due to error compensation among the large number of contained normal modes, or even just serendipity in the chosen methods. Among the directly determined components that govern the dimerization shift, the largest uncertainties stem from the harmonic contribution and the localized monomer stretching-torsion constant. Unfortunately, the factor of $1 / 2$ for the off-diagonal part implies that any error in the harmonic shift is exacerbated in the determined primed-sum contribution, which mandates accurate harmonic predictions and a stringent assessment of their uncertainties. Still, the results can be deemed satisfactory in their consistency and accuracy when considering the large number of normal modes contained in the anharmonic predictions, and the wide range of interwoven theoretical and experimental methods.

\subsection{Summary}

In this chapter, the $\mathrm{OH}$ stretching-torsion dynamics in the monomer and their transformation to the confined stretching-libration situation in the dimer have been investigated on grounds of gas-phase and matrix isolation experiments. A detailed look at the monomer situation is warranted by the 
fact that the anharmonic calculations employed herein are only able to sample a small portion of any potential energy hypersurface around a single minimum, which is in contrast to the tunneling-delocalized nature of the internal $\mathrm{OH}$ rotation in the methanol molecule. To this end, attempts at transforming the available experimental data into a similarly localized situation have been presented in Section 2.1 in order to assess the reliability of the VPT2 calculations in Section 2.3.1.

Naturally, the somewhat non-physical reduction of the torsional mode to a single-minimum localized picture introduces some ambiguities. The pure stretching transitions however obtain rather well-defined wavenumbers of $3684 \mathrm{~cm}^{-1}$ for the fundamental and $7196 \mathrm{~cm}^{-1}$ for the first overtone, resulting in a largely localization-independent diagonal anharmonicity constant of $x_{\mathrm{s}, \mathrm{s}}^{\mathrm{Mon}}=-86 \mathrm{~cm}^{-1}$. These values are compatible to within a few $\mathrm{cm}^{-1}$ with previous suggestions for the band centers by Kollipost et al. ${ }^{14}$ From the localized energy levels, an anharmonicity constant for the stretchingtorsion coupling of $x_{\mathrm{s}, \mathrm{t}}^{\mathrm{Mon}}=+15 \mathrm{~cm}^{-1}$ is proposed, but this result carries a $\pm 6 \mathrm{~cm}^{-1}$ uncertainty among the discussed localization approaches. On the quantum chemical side, VPT2 calculations perform adequately in reproducing the stretching anharmonicity. The stretching-torsion couplings are predicted with the correct positive sign, and their agreement with the localized experimental data can be deemed satisfactory in light of the localization ambiguities and the perturbational theoretical approach.

The diagonal anharmonicity content of the donor $\mathrm{OH}$ stretching mode in the methanol homodimer has previously been determined by Kollipost et $a l .,{ }^{14}$ who reported a gas-phase value of $x_{\mathrm{s}, \mathrm{s}}^{\mathrm{Dim}}=-99 \mathrm{~cm}^{-1}$. Together with a high-level harmonic prediction of $3740 \mathrm{~cm}^{-1}$, these data imply a blueshifting $+33 \mathrm{~cm}^{-1}$ correction from one half of all anharmonic couplings to other modes. Among these corrections, VPT2 results have previously predicted the stretching-libration coupling to be the most important contribution to $x_{\mathrm{s}, 1}^{\mathrm{Dim}} \approx+60 \mathrm{~cm}^{-1} .103$ This coupling element represents a dynamic "stand-off" between the stretching mode itself, which naturally further weakens the vibrating $\mathrm{O}-\mathrm{H}$ bond upon excitation, and the librational motion, which rotates the donor $\mathrm{OH}$ group out of the hydrogen-bonded arrangement and thus tendentially restore its non-bonded character. Experiments in neon matrices, which can be assumed to provide good comparability with gas-phase data, reveal this coupling to be about $+43 \mathrm{~cm}^{-1} \cdot{ }^{17}$ Still, the observable dimerization shift in the donor $\mathrm{OH}$ stretching wavenumber can be explained satisfactorily when combining all available experimental data with the averaged VPT2 predictions.

Besides this direct interpretation of the observable dimerization shift, 
the results also provide a number of insights into some quantum chemical methods and their advanced implementations in explicitly correlated local formalisms. Most interestingly, local correlation approaches have allowed to trace the demonstrable deficiency of the MP2 method to estimate the harmonic dimerization shift to an unbalanced description of the donor $\mathrm{OH}$ bond. ${ }^{15}$ Including single excitations for the bonded electron pair at the Coupled Cluster level largely alleviates this problem, and a full-scale treatment at this costly theoretical level can be approximated by carefully restricting it to a selection of important electron pairs in the LMOMO approach. At the same time, a full treatment of the methanol dimer with the explicitly correlated local Coupled Cluster $\operatorname{LCCSD}\left(\mathrm{T}^{*}\right)-\mathrm{F} 12 \mathrm{a}$ method is still competitive due to near-CBS convergence already with appropriate double-zeta VDZF12 basis set. A future LMOMO implementation of explicitly correlated local methods can be expected to combine the advantages of both approaches, thus hopefully allowing to achieve a similar accuracy for larger systems.

While the programs employed in the course of this work have not allowed for full-dimensional anharmonic treatments at the LCCSD(T0*)-F12a $a b$ initio level, estimates for the diagonal stretching anharmonicity constants in the methanol monomer and dimer have been obtained from numerical solutions of the vibrational Schrödinger equation in one-dimensional potential curves. The results are in good agreement with the experiment, as are the implied off-diagonal corrections that would be necessary to explain the remaining gap to the true experimental values. However, the VPT2 results provide similarly good results for this high-frequency motion. A variational determination of stretching-torsion and stretching-libration couplings at the LCCSD(T0*)-F12a level would therefore be most interesting to see whether the notorious VPT2 overestimation of this quantity persists. In addition, future VPT2 calculations at some higher ab initio levels with local and explicit correlation will likely provide further insight into its predictive powers for hydrogen-bonded systems. 


\section{Chapter 3}

\section{Extension to Weak and Strong Hydrogen Bonds}

In the preceding chapter, the $\mathrm{OH}$ stretching dimerization shift in the methanol dimer has been partitioned into harmonic and anharmonic components, isolating specific contributions for dedicated experimental and highlevel quantum chemical treatment. However, the analysis overall demands an effort that prohibits a routine application both in practical and theoretical terms. The overtone study by Kollipost et al. in Reference 14 extends to the ethanol and tert-butyl alcohol homodimers, for which the stronger hydrogen bonds lead to more pronounced intensity impairments in the donor $\mathrm{OH}$ stretching overtones than in methanol. In order to facilitate the observation of the weak overtone bands, and furthermore extend the anharmonicity analysis to systems of markedly different character, it is thus favorable to also go to much weaker hydrogen bonds such as $\mathrm{OH} \cdots \pi$ systems.

In this chapter, experimental and theoretical data on the methanolethene prototype will be presented. A stronger focus will be placed on the production of reliable results rather than a benchmarking of the underlying methods. Based on the results for this $\mathrm{OH} \cdots \pi$ prototype, the structural preferences in methanol-anisole complexes with competing alkoxy-O and aromatic $\pi$ acceptor sites have been elucidated, ${ }^{52}$ as detailed below in Section 3.2. Finally, the anharmonicity data collected by Kollipost et al. on ethanol and tert-butyl alcohol ${ }^{14}$ will be used for a tentative analysis of the observable dimerization shifts, highlighting some common and 

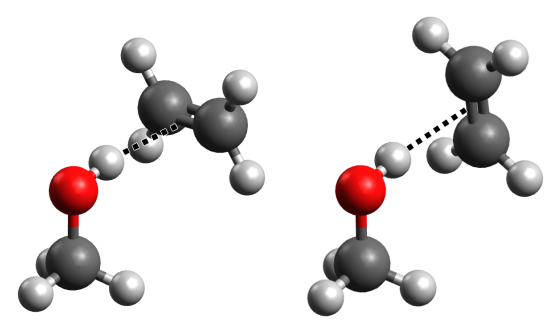

Figure 3.1: "Perpendicular" (left) and "parallel" (right) arrangements of the methanol-ethene complex, referring to the orientation of the ethene-C=C and methanol-C-O bonds. Calculations predict only the perpendicular structure to be stable.

\subsection{Methanol-Ethene}

Among the available literature on $\mathrm{OH} \cdots \pi$ hydrogen bonds, a large number of studies have employed aromatic $\pi$ acceptor sites or moieties due to their accessibility in UV resonance schemes. ${ }^{129-133}$ Further, fixation of the donor and acceptor sites in a common molecular frame aids in increasing the degree of hydrogen-bonded species, but may introduce structural strain on the hydrogen bond which distorts the energetic and spectroscopic properties. ${ }^{134-137}$

The $\mathrm{C}=\mathrm{C}$ bond in ethene provides the simplest organic $\pi$ acceptor site for hydrogen bonding. As such, it had received some interest several decades ago, mostly in conjunction with hydrogen halides as acceptor molecules. ${ }^{138-140}$ However, data on complexes with $\mathrm{OH}$ donors is scarce and most often restricted to theoretical studies, ${ }^{141-143}$ and the available experimental literature was previously limited to $\mathrm{H}_{2} \mathrm{O}$ as the donor. ${ }^{143-145}$ Concerning the methanol-ethene aggregate, only a single computational study was available up to the MP2/aVQZ level, ${ }^{141}$ predicting a "perpendicular" arrangement of the methanol donor and ethene acceptor molecules as depicted in Figure 3.1, left, and a best estimate for the electronic dissociation energy of about $3 \mathrm{kcal} \cdot \mathrm{mol}^{-1}$.

The direct FTIR absorption approach in the filet jet eliminates the need for UV chromophores, while the long optical pathway enables the detection of low concentrations of molecular aggregates. This spawned the firstever experimental characterization of the methanol-ethene dimer ${ }^{16}$ which possesses a distinct prototype character for $\mathrm{OH} \cdots \pi$ contacts due to the absence of perturbations from either intermolecular fixation or additional chromophore moieties. 


\subsubsection{Jet-FTIR Spectra}

Jet-FTIR spectra of different methanol-ethene mixtures are depicted in Figure 3.2 in the $\mathrm{OH}$ stretching fundamental and overtone regions, reproduced here from Reference 16. The strong black trace in the fundamental region corresponds to a methanol:ethene ratio of about 1:20, the lighter trace to a 1:7 ratio. The latter mixture was further used for the overtone measurements. As in the methanol spectra in Section 2.2.1, the wavenumber scale of the $\mathrm{OH}$ stretching overtone region is compressed by a factor of 2 and centered to the methanol monomer band to visualize the increase in dimer stretching anharmonicity.

In the fundamental region, a prominent band is visible at $3641 \mathrm{~cm}^{-1}$, with a number of smaller bands congesting the region towards the methanol homodimer. To ascertain the assignment of the least red-shifted signal to the methanol-ethene dimer, a concentration series was recorded by subsequently increasing the relative methanol amount in the sample mixture. The resulting spectra are shown in the bottom part of Figure 3.2 after scaling to the $3641 \mathrm{~cm}^{-1}$ band, with the strongest trace corresponding to the 1:20 ratio shown above in the fundamental region. The distinctly different behavior of the unspecific ">ME" bands with the decreasing relative ethene concentration is in line with a previous study on $\mathrm{OH} \cdots \pi$ hydrogen bonds to bulky alkene acceptors. ${ }^{146}$ These signals were thus assigned to a multitude of possible mixed structures beyond the dimer, and will not be discussed here any further. In addition, the dimer band itself shows a slight asymmetry in the spectra, which may be attributable to residual rotational structure. In relation to the localized monomer band center of $3684 \mathrm{~cm}^{-1}$ (see Section 2.1), the dimerization shift now amounts to only $43 \mathrm{~cm}^{-1}$, which is the first of several indications for a remarkably weak hydrogen bond.

In line with expectations, the combined fundamental and overtone spectra displayed in Figure 3.2 indicate a much smaller increase in diagonal $\mathrm{OH}$ stretching anharmonicity in the $\mathrm{OH} \cdots \pi$ hydrogen bond when compared to its $\mathrm{OH} \cdots \mathrm{O}$ counterpart in the methanol homodimer. From the $3641 \mathrm{~cm}^{-1}$ fundamental and $7105 \mathrm{~cm}^{-1}$ overtone stretching band wavenumbers, a diagonal anharmonicity of about $x_{\mathrm{s}, \mathrm{s}}^{\mathrm{Dim}}=-89(1) \mathrm{cm}^{-1}$ is derived for methanolethene, close to the methanol monomer value of $c a .-86 \mathrm{~cm}^{-1}$ and distinctly smaller than in the methanol homodimer with $-99 \mathrm{~cm}^{-1}$. At the same time, the overtone/fundamental intensity ratio is found to be only 170(70), again quite moderate in comparison to the methanol homodimer value of 320(90). While both effects testify to the weakness of the $\mathrm{OH} \cdots \pi$ contact, the absolute overtone intensity does not show a decisive advantage to the pure methanol spectra in Figure 3.2 in terms of facilitating its observation in the filet-jet 

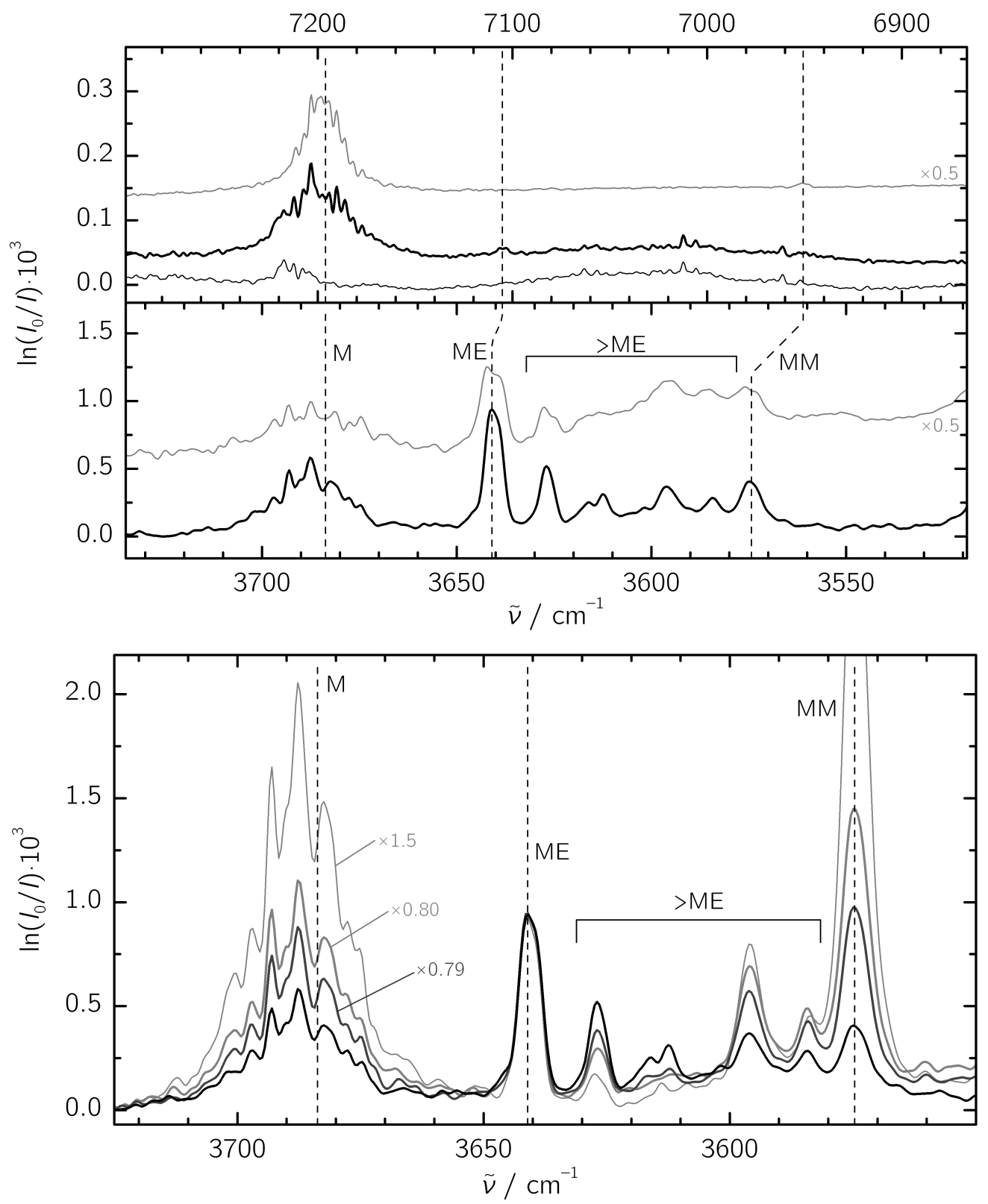

Figure 3.2: Top: Jet-FTIR spectra of different methanol-ethene mixtures (see also Ref. 16) in the $\mathrm{OH}$ stretching overtone and fundamental regions (upper and lower panels, resp.). Annotated are the band centers of the methanol monomer ("M", from Section 2.1), methanol homodimer ("MM"), and methanol-ethene clusters (dimer "ME", larger aggregates ">ME"). Bottom: Concentration series in the $\mathrm{OH}$ stretching fundamental region (from Ref. 16), scaled to the "ME" dimer band by the annotated factors. 
experiment. This is a direct effect of the reduced concentration of the $\mathrm{OH}$ chromophore in the mixed methanol:ethene expansions, and may possibly be aggravated by a lower energetic propensity for the formation of the weaker $\mathrm{OH} \cdots \pi$ clusters.

\subsubsection{Quantum Chemical Calculations}

Due to the comparable size of methanol-ethene and the methanol homodimer, the same computational methods can readily be applied with only minor differences in resource demand. Where supported, exploiting the apparent $\mathrm{C}_{\mathrm{s}}$ symmetry of the methanol-ethene structure (see Figure 3.1) further aids in the calculations. Based on the extensive robustness studies in the previous chapter, only a selection of methods was applied to the methanolethene problem with a more production-centric focus. In the present case however, no direct experimental information on the stretching-libration coupling constant is available, and the VPT2 predictions given below are so far the only available theoretical estimates for these contributions.

\section{Local and Canonical Harmonic Results}

Table 3.1 contains a number of harmonic results for methanol-ethene. ${ }^{16}$ The LCCSD(T0*)-F12a(int)/VDZ-F12 level of theory was directly chosen as a benchmark for dissociation energies and harmonic wavenumbers, since the respective methanol homodimer results did not show substantial variations from the triple- and quadruple-zeta levels.

Across all calculations, the lowest-frequency vibration represents a torsional motion of the ethene molecule around the axis of the $\mathrm{OH} \cdots \pi$ hydrogen bond. In essence, the associated normal coordinate corresponds to an interconversion between the "perpendicular" and "parallel" arrangements depicted in Figure 3.1. This vibration is occasionally predicted at nearvanishing or imaginary wavenumbers in the "perpendicular" structures, as indicated in Table 3.1, and adopts values of up to $20 \mathrm{~cm}^{-1}$ in other cases. For the "parallel" structure, all calculations predict imaginary wavenumbers, suggesting that this arrangement is indeed unstable. Even for the correct "perpendicular" structure however, effects of imaginary ethene-torsion wavenumbers on the $\mathrm{OH}$ stretching mode can be assumed to be small, seeing that even a full switchover between both structures leads to only minute variations at the theoretical levels included in Table 3.1. Nevertheless, a truly robust method should still be able to provide a realistic description of this motion, which can thus be considered as a sensitive accuracy check. 
Table 3.1: Collection of harmonic predictions for the methanol-ethene dimer, "parallel" and "perpendicular" geometries (see Fig. 3.1): dissociation energies $D_{0}$ and $D_{\mathrm{e}}$ in $\mathrm{kJ} \mathrm{mol}^{-1}$; and wavenumbers $\omega$ for $\mathrm{O}-\mathrm{H}$ stretching and torsion/libration modes (subscripts "s", " $\mathrm{t}$ " and "l", resp.) in the monomer and dimer, together with corresponding dimerization shifts, in $\mathrm{cm}^{-1}$.

\begin{tabular}{|c|c|c|c|c|c|c|c|}
\hline & $D_{0}\left(D_{\mathrm{e}}\right)$ & $\omega_{\mathrm{s}}^{\text {Mon }}$ & $\omega_{\mathrm{s}}^{\operatorname{Dim}}$ & $-\Delta \omega_{\mathrm{s}}$ & $\omega_{\mathrm{t}}^{\mathrm{Mon}}$ & $\omega_{1}^{\operatorname{Dim}}$ & $\Delta \omega_{1 / t}$ \\
\hline \multicolumn{8}{|c|}{ perpendicular } \\
\hline B3LYP-D3/VTZ & $12.6(15.9)$ & 3829 & 3776 & 53 & 306 & 425 & 119 \\
\hline B2PLYP-D3/VTZ & $11.7(15.1)$ & 3858 & 3805 & 53 & 307 & 431 & 125 \\
\hline MP2/VTZ & $11.1(14.6)$ & 3882 & 3823 & 60 & 309 & 448 & 139 \\
\hline $\mathrm{MP} 2 / \mathrm{aVTZ}^{\mathrm{a}}$ & $11.2(14.7)$ & 3860 & 3790 & 70 & 290 & 463 & 173 \\
\hline $\mathrm{LMP}^{\text {/ aVTZ }}{ }^{\mathrm{a}}$ & $8.5(11.7)$ & 3856 & 3793 & 64 & 293 & 446 & 152 \\
\hline SCS-LMP2 / aVTZ ${ }^{\mathrm{a}}$ & $6.3(9.3)$ & 3852 & 3809 & 43 & 293 & 428 & 135 \\
\hline $\mathrm{LCCSD}(\mathrm{T} 0) / \mathrm{aVTZ}{ }^{\mathrm{a}}$ & $7.7(11.2)$ & 3828 & 3789 & 39 & 293 & 436 & 142 \\
\hline LCCSD(T0)(int):LMP2 / aVTZ ${ }^{\mathrm{b}}$ & $6.7(10.1)$ & 3836 & 3797 & 39 & 294 & 434 & 139 \\
\hline LCCSD(T0*)-F12a(int)/VDZ-F12 & $7.7(10.9)$ & 3862 & 3817 & 45 & 294 & 436 & 142 \\
\hline \multicolumn{8}{|c|}{ parallel } \\
\hline $\mathrm{MP} 2 / \mathrm{aVTZ}^{\mathrm{a}}$ & $11.2(14.8)$ & 3860 & 3788 & 72 & 290 & 480 & 190 \\
\hline $\mathrm{LMP} / \mathrm{aVTZ}{ }^{\mathrm{a}}$ & $8.6(11.7)$ & 3856 & 3791 & 66 & 293 & 459 & 166 \\
\hline SCS-LMP2 / aVTZ & $6.3(9.3)$ & 3852 & 3808 & 44 & 293 & 438 & 145 \\
\hline $\mathrm{LCCSD}(\mathrm{T} 0) / \mathrm{aVTZ}$ & $8.0(11.3)$ & 3828 & 3790 & 38 & 293 & 441 & 147 \\
\hline
\end{tabular}

${ }^{\text {a }}$ Low or imaginary wavenumbers in lowest normal mode, see text for details.

${ }^{\mathrm{b}}$ LMOMO calculation, see text for details.

c Using default electronic convergence criteria in MOLPRO.

Comparison of the harmonic results to the methanol dimer reveals several striking measures for the weakness of the $\mathrm{OH} \cdots \pi$ interaction at hand. First, the harmonic $\mathrm{OH}$ stretching dimerization shift is drastically reduced, but with similar tendencies for overestimation in MP2 and DFT. Using the explicitly correlated local Coupled Cluster result of $45 \mathrm{~cm}^{-1}$ as a benchmark, canonical and local MP2 methods notoriously exaggerate this quantity by up to $15 \mathrm{~cm}^{-1}$-or $56 \%$-but again benefit from applying SCS. In line with the reduced weakening of the donor $\mathrm{O}-\mathrm{H}$ bond, the blue-shifting influence of the $\mathrm{OH} \cdots \pi$ contact on the monomer $\mathrm{OH}$ torsion wavenumber is also much smaller than in the homodimeric $\mathrm{OH} \cdots \mathrm{O}$ contact. This can be readily explained with the $\pi$ electron cloud of the ethene molecule being a much weaker and spatially more diffuse acceptor site than an oxygen lone pair. Perhaps even more interesting is the fact that the best harmonic estimates, 
in particular at the $\operatorname{LCCSD}\left(\mathrm{T0} 0^{*}\right)-\mathrm{F} 12 \mathrm{a}(\mathrm{int}) / \mathrm{VDZ}-\mathrm{F} 12$ level, predict the harmonic dimerization shift to almost coincide with the experimental value. The subsequent anharmonic corrections therefore appear to largely compensate each other, which suggests that for sufficiently weak hydrogen-bonding interactions, the observable dimerization shift might already be adequately explained in a harmonic picture.

Even despite the moderate size of the molecular system, full-scale LCCSD(T0)(int) frequency calculations are still rather costly due to the lack of analytical gradients. However, the benchmark calculations for the methanol dimer in Section 2.3 suggest that such a treatment can be adequately approximated by LMOMO methods as long as the $\mathrm{C}-\mathrm{O}$ bond in the methanol donor is included in the high-level region. A calculation similar to the "LMOMO+C-O(int)" scheme in Table 2.5 was thus carried out for methanol-ethene, where the full ethene acceptor molecule and all intermolecular excitations were kept at the Coupled Cluster level; the results are included in Table 3.1 as "LCCSD(T0)(int):LMP2". As in the methanol homodimer, the harmonic $\mathrm{OH}$ stretching wavenumber in methanol-ethene is somewhat lower than the explicitly correlated reference value, while the dimerization shift falls somewhat short of it.

\section{VPT2 Calculations}

As for the methanol dimer, VPT2 calculations were conducted at the B3LYPD3, B2PLYP-D3 and MP2 levels using the VTZ basis set. Again, inconsistencies pertaining to the chosen starting geometries and checkpoint files were found from repeated calculations. ${ }^{17}$ Even worse, the general usefulness of the B3LYP-D3 results is somewhat doubtful, since a number of lowfrequency vibrations persistently obtain sizable imaginary frequencies of up to $200 \mathrm{i} \mathrm{cm}^{-1}$ in the anharmonic picture. These instabilities were found to be smaller at the B2PLYP-D3 level.

The results assembled in Table 3.2 demonstrate that the chosen methods generally provide adequate predictions for diagonal stretching anharmonicity constants, $x_{\mathrm{s}, \mathrm{s}}^{\mathrm{Dim}}$, again with slight DFT tendencies for overestimation as in the methanol dimer. Due to the lack of experimental data on the stretchinglibration combination band, the VPT2 calculations so far provide the only estimates for the related coupling element, $x_{\mathrm{s}, 1}^{\mathrm{Dim}}$. These predictions, averaging some $+16(2) \mathrm{cm}^{-1}$, are again comparable among the three methods, and significantly smaller in magnitude than in the methanol case $\left(+59(1) \mathrm{cm}^{-1}\right)$. At the DFT level, the remaining primed-sum terms now show such large instabilities that their predictions are practically unusable; only the MP2 value of $-4 \mathrm{~cm}^{-1}$ is again robust. 
Table 3.2: VPT2-anharmonic results $\left(\right.$ in $\mathrm{cm}^{-1}$ ) for the methanol monomer and the "perpendicular" methanol-ethene dimer on different levels of theory (see also Refs. 16,17; monomer repeated from Sec. 2.3.1): diagonal $\mathrm{OH}$ stretching anharmonicity constants $\left(x_{\mathrm{s}, \mathrm{s}}\right)$; stretching-torsion and -libration coupling constants $\left(x_{\mathrm{s}, \mathrm{t}}\right.$ and $\left.x_{\mathrm{s}, 1}\right)$; and remaining primed-sum terms as per Eqs. (2.5) and (2.6). Also given are experimental data, using the monomer $x_{\mathrm{s}, \mathrm{t}}$ reference from Sec. 2.1 .

\begin{tabular}{lccccccc}
\hline & \multicolumn{3}{c}{ Monomer } & \multicolumn{3}{c}{ Dimer } \\
\cline { 2 - 3 } \cline { 6 - 8 } & $x_{\mathrm{s}, \mathrm{s}}$ & $x_{\mathrm{s}, \mathrm{t}}$ & $\sum^{\prime} x_{\mathrm{s}, j}$ & & $x_{\mathrm{s}, \mathrm{s}}$ & $x_{\mathrm{s}, 1}$ & $\sum^{\prime} x_{\mathrm{s}, j}$ \\
\hline B2PLYP-D3/VTZ & -86 & +4 & -28 & & -91 & +14 & \\
B3LYP-D3/VTZ & -87 & +3 & -26 & -92 & +18 & \\
MP2/VTZ & -83 & +9 & -30 & -88 & +17 & -4 \\
exp. & $-86(1)$ & $+15(6)$ & & $-89(1)$ & & \\
\hline
\end{tabular}

\section{1-D Numerical Solutions}

As before, a one-dimensional potential curve was calculated in the $\mathrm{OH}$-stretching normal mode for methanol-ethene at the $\operatorname{LCCSD}\left(\mathrm{T} 0^{*}\right)$ F12a(int)/VDZ-F12 level of theory, and fitted with the modified Morse potential given in Equation (2.7). ${ }^{16}$ Building numerical solutions of the vibrational Schrödinger equation yielded fundamental and overtone transition wavenumbers of 3641 and $7108 \mathrm{~cm}^{-1}$, respectively, with a diagonal anharmonicity constant of $x_{\mathrm{s}, \mathrm{s}}^{\mathrm{Dim}}=-88 \mathrm{~cm}^{-1}$. The latter is again in good agreement with the experimental value of $-89 \mathrm{~cm}^{-1}$, but more interestingly, the transition wavenumbers now also closely match the observed ones at 3641 and $7105 \mathrm{~cm}^{-1}$. Conversely, the methanol calculations in Section 2.3 .1 have shown similar agreement in the anharmonicity constant, but demonstrate a distinct underestimation of the transition wavenumbers in relation to the experiment due to the neglect of overall blue-shifting anharmonic couplings. In closely matching the experimental values, the calculated methanol-ethene wavenumbers thus suggest that the off-diagonal contributions largely cancel out. If possible fitting errors and shortcomings of the perturbational anharmonic method are taken into account, the MP2 VPT2 prediction of a $\frac{1}{2} \sum x_{\mathrm{x}, i}^{\mathrm{Dim}}=+7 \mathrm{~cm}^{-1}$ off-diagonal blue shift from Table 3.2 is moderately compatible with the 1-D result. 


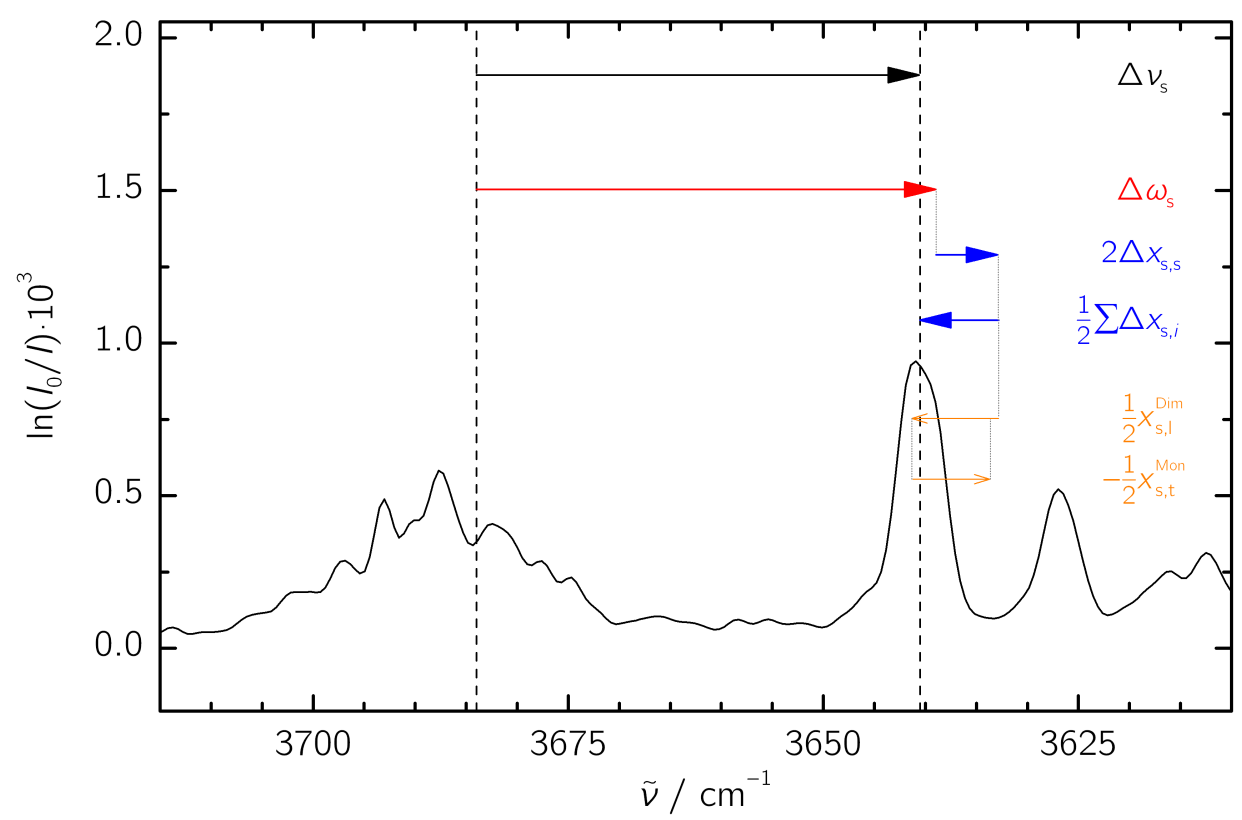

Figure 3.3: Visual dissection of the overall methanol-ethene $\mathrm{OH}$ dimerization red shift (black) into its harmonic (red) and anharmonic (blue) components, with the stretching-torsion/libration couplings (orange) separated from the latter.

\subsubsection{OH Dimerization Red Shift}

Among the different contributions to the observable dimerization shift (Table 3.3),

$$
-\Delta \tilde{v}_{\mathrm{s}}=-\Delta \omega_{\mathrm{s}}-2 \Delta x_{\mathrm{s}, \mathrm{s}}-\frac{1}{2} \sum_{i \neq \mathrm{s}} \Delta x_{\mathrm{s}, i},
$$

the harmonic and diagonal anharmonic components are now fairly wellestablished, and the analysis again hinges on the off-diagonal contributions. The jet-FTIR experiments in Section 3.1.1 indicate a dimerization shift of $-\Delta \tilde{v}_{\mathrm{s}}=43(1) \mathrm{cm}^{-1}$ and a contained diagonal harmonic contribution of $-2 \Delta x_{\mathrm{s}, \mathrm{s}}=+7(2) \mathrm{cm}^{-1}$. Together with the best harmonic estimate of $-\Delta \omega_{\mathrm{s}}=45 \mathrm{~cm}^{-1}$ from Table 3.1 , the effective blue-shifting correction supplied by the halved off-diagonal sum in Equation (3.1) must be about $-\frac{1}{2} \sum \Delta x_{\mathrm{s}, i}=-8 \mathrm{~cm}^{-1}$. The dissection of the overall dimerization shift into its various components is visualized in Figure 3.3.

In contrast to the methanol homodimer, the lack of dedicated experimental stretching-libration data for the methanol-ethene dimer now only war- 
Table 3.3: Summary of harmonic and anharmonic terms (in $\mathrm{cm}^{-1}$ ) in the localized methanol monomer ("M") and methanol-ethene dimer ("ME"), together with negative dimer-monomer differences ("- $\Delta$ ") in all quantities. Also given are the methanol homodimer data ("MM") from Tab. 2.9. The overall off-diagonal $\left(\sum x_{\mathrm{s}, i}\right)$ and primed-sum $\left(\sum^{\prime} x_{\mathrm{s}, j}\right)$ values are deduced from the preceding data, and the primed sums are compared to VPT2 predictions in the last line.

\begin{tabular}{lrrr|rr}
\hline & $\mathrm{M}$ & $\mathrm{ME}$ & $-\Delta$ & $\mathrm{MM}$ & $-\Delta$ \\
\hline$\omega_{\mathrm{s}}$ & 3862 & 3817 & +45 & 3740 & +121 \\
$\tilde{v}_{\mathrm{S}}$ & 3684 & 3641 & +43 & 3575 & +109 \\
$2 x_{\mathrm{s}, \mathrm{s}}$ & -171 & -178 & +7 & -198 & +27 \\
$\Rightarrow \frac{1}{2} \sum x_{\mathrm{s}, i}$ & -7 & +2 & -9 & +33 & -40 \\
$\frac{1}{2} x_{\mathrm{s}, 1 / \mathrm{t}}$ & +8 & +8 & 0 & +22 & -14 \\
$\Rightarrow \frac{1}{2} \sum^{\prime} x_{\mathrm{s}, j}$ & -15 & -6 & -9 & +11 & -26 \\
\multicolumn{1}{c}{$\mathrm{VPT} 2:$} & -14 & -2 & -12 & +8 & -22 \\
\hline
\end{tabular}

rants a separation of the off-diagonal contribution into the overall monomer and dimer sums, i.e.

$$
\sum \Delta x_{\mathrm{s}, i}=\sum x_{\mathrm{s}, i}^{\mathrm{Dim}}-\sum x_{\mathrm{s}, i}^{\mathrm{Mon}}
$$

The monomer data given in Table 2.6 are used here as an anchor point to deduce the anharmonicity situation in methanol-ethene, seeing their robustness and the satisfying consistency they have provided for the homodimer analysis. Specifically, the full off-diagonal monomer terms add a blue-shifting contribution of about $-\frac{1}{2} \sum x_{\mathrm{s}, i}^{\mathrm{Mon}}=7 \mathrm{~cm}^{-1}$. Closing the remaining gap to the methanol-ethene experiments consequentially requires the corresponding dimer terms to contribute only a $-\frac{1}{2} \sum x_{\mathrm{s}, i}^{\text {Dim }}=+2 \mathrm{~cm}^{-1}$ blue shift. To achieve this, the primed dimer sum has to mostly negate the stretching-libration coupling, predicted at the VPT2 level to be $+16(2) \mathrm{cm}^{-1}$; i.e., $\frac{1}{2} \sum^{\prime} x_{\mathrm{s}, j}^{\mathrm{Dim}}=-6 \mathrm{~cm}^{-1}$. While mostly consistent with the MP2 VPT2 prediction of $-4 \mathrm{~cm}^{-1}$ (see Table 3.2), this would be in contrast to the methanol dimer, for which the calculations predict a distinctly positive value (Table 3.3).

Overall, the results suggest that the off-diagonal anharmonic contributions in methanol-ethene are much smaller than in the methanol homodimer. However, the rather subtle compensation effects among the diagonal and off-diagonal contributions to the dimerization shift now cause the 

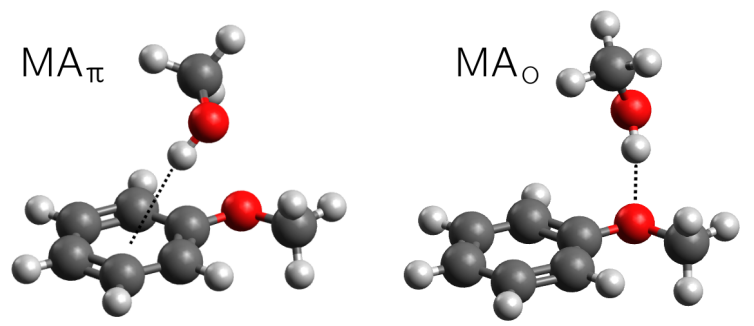

Figure 3.4: Methanol-anisole structures of $\mathrm{OH} \cdots \pi$ ("MA ${ }_{\pi}$ ", left) and $\mathrm{OH} \cdots \mathrm{O}$ ("MA ${ }_{\mathrm{O}}$ ", right) hydrogen bonding motifs.

analysis to be dominated by their error bars. Obtaining an experimental stretching-libration coupling constant would be helpful in somewhat driving these errors back. Still, the VPT2 calculations suggest a much smaller impact of the $\mathrm{OH} \cdots \pi$ hydrogen bond on this quantity, which would be consistent with other indications of a weak intermolecular contact.

\subsection{Concurrent Docking Motifs in Methanol- Anisole Dimers}

The given findings on the $\mathrm{OH} \cdots \pi$ hydrogen bond in methanol-ethene can be related to a study on the interactions of methanol with anisole (methoxybenzene). ${ }^{52}$ This molecule offers two potential acceptor sites for hydrogen bonding, as depicted in Figure 3.4: the etheric oxygen atom and the extended $\pi$ system of the aromatic ring.* Preliminary quantum chemical calculations had suggested the two binding motifs to yield approximately isoenergetic structures, with a tendency in MP2 calculations to favor the $\pi$-bonded option. Direct IR-absorption measurements were thus conducted in the filet jet to verify this close energetic match.

\subsubsection{Spectroscopic Evidence}

A collection of jet-FTIR spectra of methanol:anisole mixtures is shown in Figure 3.5, reproduced here from Reference $52 .^{\dagger}$ In the $\mathrm{OH}$-stretching funda-

\footnotetext{
*A second $\mathrm{OH} \cdots \pi^{\prime}$ structure was found in Ref. 52 , but will not be considered here due to the presumably facile conversion to the more stable $\mathrm{OH} \cdots \pi$ structure.

${ }^{\dagger}$ Note that the intensity scale of the "overtone" panel in Reference 52 is wrong: the given axis labels should be multiplied by $4.342=10 / \ln 10$. The $\mathrm{OH}$ and $\mathrm{OD}$ fundamental regions are labeled correctly.
} 
mental region (middle panel of Figure 3.5), a dominant signal at $3598 \mathrm{~cm}^{-1}$ is observed between the methanol monomer and homodimer bands, corresponding to a dimerization red shift of $86 \mathrm{~cm}^{-1}$ relative to the localized monomer band center. The shift and strength of this signal primarily suggest an $\mathrm{OH} \cdots \mathrm{O}$ origin, but at this point, an accidental overlap with a particularly strong $\mathrm{OH} \cdots \pi$ band could not be ruled out. Further spectroscopic information was thus sought through overtone measurements (top panel of Figure 3.5), since the presumably different anharmonicity contents of the $\mathrm{OH} \cdots \mathrm{O}$ and $\mathrm{OH} \cdots \pi$ vibrations would drive any overlapping bands apart.

In the overtone spectra, a band of mixed dimer origin is visible at $7005 \mathrm{~cm}^{-1}$, revealing a diagonal anharmonicity constant of $x_{\mathrm{s}, \mathrm{s}}^{\mathrm{Dim}}=-96 \mathrm{~cm}^{-1}$. A re-evaluation of the fundamental/overtone ratios for the methanol-anisole band in question and its homodimer pendant suggest them to be on order of 430(150) and 530(250), respectively. The latter value is larger than the one 320(90) result determined previously by Kollipost et al. ${ }^{14}$ This may be an effect of the high-wavenumber shoulder on the homodimer fundamental band that is visible in Figure 3.5, and the lower signal-to-noise ratio in the recorded spectra. Still, both the intensity ratio and anharmonicity constant deduced from these overtone measurements hint towards an $\mathrm{OH} \cdots \mathrm{O}$ character of the methanol-anisole signal in question, and the according bands in Figure 3.5 are thus labeled "MAO".

As an additional tactic to rule out accidental $\mathrm{OH} \cdots \mathrm{O} / \mathrm{OH} \cdots \pi$ band overlap, spectra of methanol-OD:anisole structures were recorded at a slightly improved $1.5 \mathrm{~cm}^{-1}$ resolution, shown in the bottom panel of Figure 3.5 with a $\sqrt{2}$-fold expanded wavenumber scale. The isotope effect should again lead to a separation of any offending bands, but no such effect was found in the spectra.

At a total of 210 co-added jet scans in the $\mathrm{OH}$ stretching fundamental region, a weak band at $3629 \mathrm{~cm}^{-1}$ with a corresponding red shift of $55 \mathrm{~cm}^{-1}$ from the methanol monomer became apparent. The inset in the middle panel of Figure 3.5 furthermore shows the $3632 \mathrm{~cm}^{-1} \mathrm{OH}$ stretching band of the methanol-toluene dimer as a template for the interaction of a methanol donor molecule with a slightly electron-enriched aromatic $\pi$ system. The proximity to this signal suggests the subtle band in the methanol:anisole mixture to stem from the $\mathrm{OH} \cdots \pi$-bonded " $\mathrm{MA}_{\pi}$ " structure (see Figure 3.4). Considering the relative overtone intensities in methanol-ethene and the $\mathrm{MA}_{O}$ structure as limiting cases, the $\mathrm{OH} \cdots \pi$ bond in the proposed $\mathrm{MA}_{\pi}$ aggregate can be assumed to induce a relative attenuation factor between 200 and 500 in the corresponding overtone band, effectively disallowing its observation in the given spectra. Still, the assignment of the fundamental band is fairly robust in light of the comparable toluene-acceptor signal 


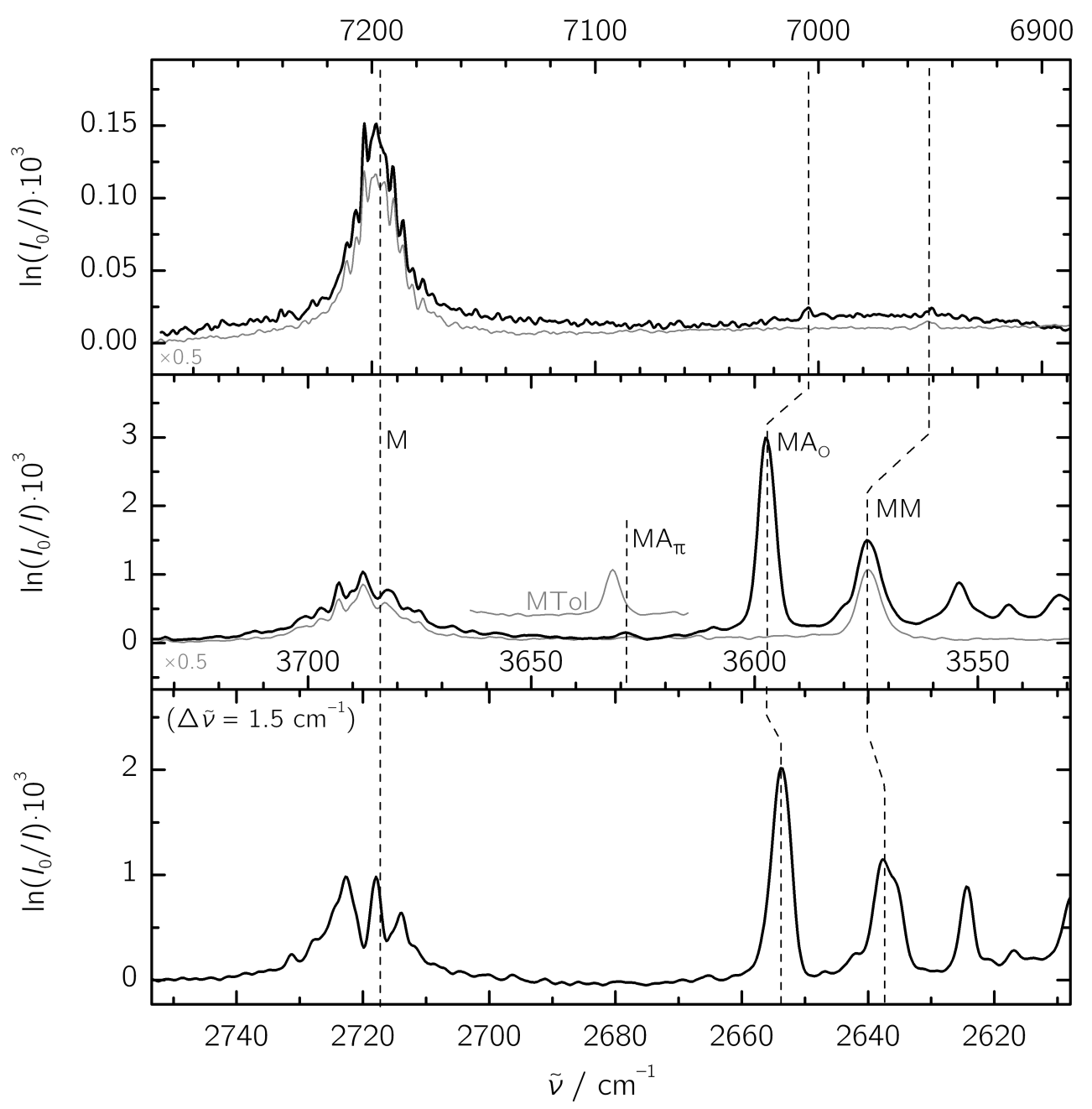

Figure 3.5: Jet-FTIR spectra of methanol:anisole mixtures in the $\mathrm{OH}$ stretching fundamental (middle) and overtone regions (top panel), and of a methanol-OD:anisole mixture in the OD stretching region (bottom panel), see also Ref. 52. "M" and "MM" denote the methanol monomer and homodimer, "MAo" and "MA" the methanol-anisole bands (see Fig. 3.4); the "MTol" inset shows the methanol-toluene dimer band. Grey traces are pure methanol spectra from Fig. 2.6, intensity-scaled by 0.5 . 
Table 3.4: Collection of electronic and zero-point corrected dissociation energies $D_{\mathrm{e}}$ and $D_{0}$ for $\mathrm{MA}_{\mathrm{O}}$ and $\mathrm{MA}_{\pi}$ structures, all in $\mathrm{kJ} \mathrm{mol}^{-1}$. The $\Delta_{\pi-\mathrm{O}}$ differences give the relative energy of the $\mathrm{MA}_{O}$ conformer with respect to $\mathrm{MA}_{\pi}$ (negative values indicating a preference for the O-bonded structure).

\begin{tabular}{|c|c|c|c|c|c|c|}
\hline & \multicolumn{2}{|l|}{$\mathrm{MA}_{\mathrm{O}}$} & \multicolumn{2}{|l|}{$\mathrm{MA}_{\pi}$} & \multicolumn{2}{|l|}{$\Delta_{\pi-\mathrm{O}}$} \\
\hline & $D_{0}$ & $D_{\mathrm{e}}$ & $D_{0}$ & $D_{\mathrm{e}}$ & $D_{0}$ & $D_{\mathrm{e}}$ \\
\hline MP2/aVTZ & & 28.7 & & 28.6 & & -0.1 \\
\hline $\mathrm{MP} 2 / \mathrm{aVQZ}+\Delta \mathrm{CC}^{\mathrm{a}, \mathrm{b}, \mathrm{c}}$ & & 25.0 & & 23.0 & & -2.0 \\
\hline LMP2/aVTZ & 18.1 & 22.4 & 18.2 & 21.6 & +0.2 & -0.7 \\
\hline SCS-LMP2/aVTZ & 14.3 & 18.6 & 13.6 & 16.9 & -0.7 & -1.7 \\
\hline LMP2-F12/VTZ-F12 & & 23.4 & & 22.3 & & -1.1 \\
\hline SCS-LMP2-F12/VTZ-F12 & & 19.3 & & 17.4 & & -2.1 \\
\hline LMP2-F12/VTZ-F12 & & 22.9 & & 21.7 & & -1.1 \\
\hline SCS-LMP2-F12/VTZ-F12 & & 19.4 & & 17.3 & & -2.1 \\
\hline
\end{tabular}

\footnotetext{
a See Ref. 52.

b Including Coupled Cluster corrections.

c B2PLYP-D3BJ/aVTZ structures, provided by J. Altnöder.

d SCS-LMP2/aVTZ structures.
}

and the strong indications for an $\mathrm{OH} \cdots \mathrm{O}$ origin of the competing lowerwavenumber dimer. In line with the assignments, harmonic quantum chemical calculations further suggested that the zero-point stabilization due to the aforementioned H/D substitution should be more pronounced for the $\mathrm{MA}_{O}$ than for the $\mathrm{MA}_{\pi}$ structure, which is reflected in the spectra by the absence of an equivalent observable OD $\cdots \pi$ signal.

The vastly different intensities of the assigned $\mathrm{MA}_{O}$ and $\mathrm{MA}_{\pi}$ signals reveal a much higher propensity for the formation of the $\mathrm{OH} \cdots \mathrm{O}$-bonded structure, even when taking the inherent effects in the extinction coefficients into account. In predicting an energetic competitiveness of both motifs, many quantum chemical calculations clearly contradict this finding (see Table 3.4). The MP2/aVTZ results from Reference 52 can be seen as an example for such a "misbalanced" method for the energy difference. This problem was overcome by calculating MP2/aVQZ dissociation energies at B2PLYPD3BJ/aVTZ structures, and adding Coupled Cluster corrections (" $\Delta \mathrm{CC}$ in Table 3.4). ${ }^{52}$ Alternatively, switching from canonical to local correlation already drives the two conformers apart in the correct direction, and applying 
SCS further widens this gap. In addition, the dissociation energies of the B2PLYP-D3BJ/aVTZ and SCS-LMP2/aVTZ structures were re-calculated including explicit correlation. In both cases, SCS-LMP2-F12/VTZ-F12 yields an energy gap which is close to the best canonical predictions, at the expectable expense of underestimated absolute dissociation energies. However, the lack of structure optimizations at the explicitly correlated levels renders these results somewhat tentative. In addition, re-calculating the absolute dissociation energies at local Coupled Cluster levels, preferably including explicit correlation, would provide more stringent benchmarks than the empirical MP2/aVQZ $+\Delta \mathrm{CC}$ scheme. Still, it appears that local correlation-and possibly also SCS-aid in predicting the correct structural preference in this system.

By appropriate substitution of the aromatic ring, the energetic scale can be influenced to some degree, but a general tendency of many popular quantum chemical methods to over-stabilize the $\pi$-bonded structures persists for many substitution patterns. ${ }^{147}$

\subsubsection{Harmonic and Anharmonic Predictions}

The smaller experimental dimerization shift of the $\mathrm{MA}_{\mathrm{O}}$ band in comparison to the methanol dimer band $\left(86 \mathrm{~cm}^{-1}\right.$ vs. $\left.109 \mathrm{~cm}^{-1}\right)$ and the slightly smaller diagonal anharmonicity constant $\left(-96\right.$ vs. $\left.-99 \mathrm{~cm}^{-1}\right)$ suggest that the $\mathrm{OH} \cdots \mathrm{O}$ hydrogen bond in methanol-anisole is somewhat weaker than in the methanol homodimer. Conversely, the dimerization shift alone in the $\mathrm{MA}_{\pi}$ structure might indicate a slightly stronger contact than in the methanol-ethene prototype $\left(55 \mathrm{~cm}^{-1}\right.$ vs. $\left.43 \mathrm{~cm}^{-1}\right)$. Without experimental data on the diagonal stretching anharmonicity however, this remains speculative. A number of harmonic and anharmonic B3LYP estimates, including comparative calculations on the methanol homodimer, are presented in Table $3.5 .^{\ddagger}$ No stability tests were carried out for these systems, but based on the preceding methanol homodimer and methanol-ethene findings, the $x_{\mathrm{s}, \mathrm{s}}$ and $x_{\mathrm{s}, 1}$ coupling constants from VPT2 calculations can be assumed to be robust even if general numerical inconsistencies are present.

The B3LYP data again show a certain tendency to overestimate harmonic $\mathrm{OH} \cdots \mathrm{O}$ dimerization shifts. In the $\mathrm{MA}_{\pi}$ case however, the results now agree with SCS-LMP2 on the harmonic $\mathrm{OH} \cdots \pi$ shift, contrary to the behavior in methanol-ethene (see Table 3.1) where B3LYP again slightly exaggerated

\footnotetext{
¥The B3LYP calculations on methanol-anisole predict the librational motion to be distributed across two normal modes. The $x_{\mathrm{s}, 1}^{\mathrm{Dim}}$ data in Tab. 3.5 therefore reflect the sums of both corresponding values.
} 
Table 3.5: Harmonic and anharmonic estimates $\left(\mathrm{in}^{-1}\right.$ ) for the methanol monomer, homodimer, and two methanol-anisole structures at various levels of theory. The stretching-libration couplings $x_{\mathrm{s}, 1}$ in the methanol-anisole structures are the sums of two values each due to mode mixing; see the text for details.

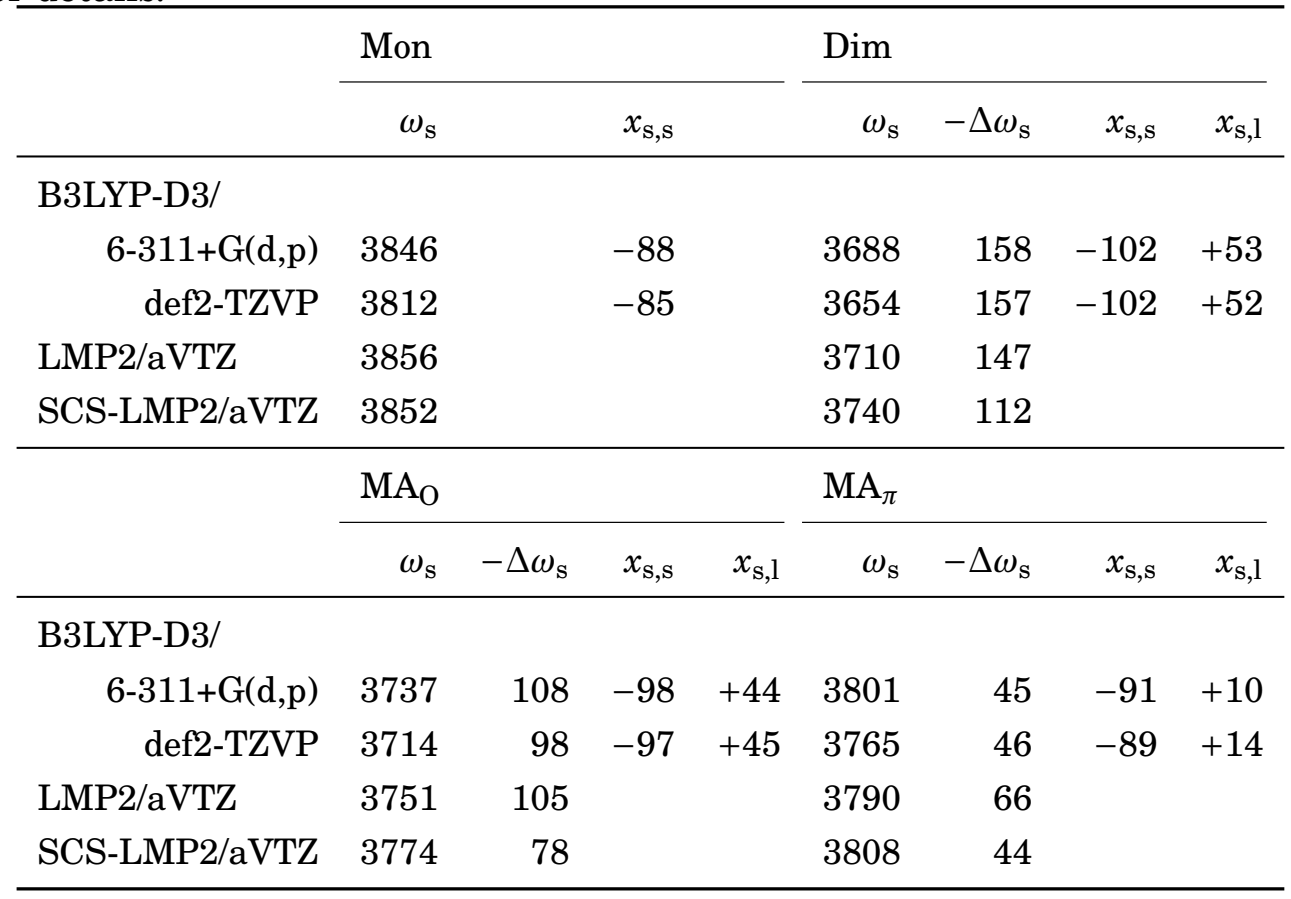


this quantity. However, the lack of higher-level references does not allow definite statements about the quality of either method. In terms of the anharmonic predictions, the data show a qualitative correlation between the harmonic red shift, the diagonal anharmonicity content, and the magnitude of the stretching-libration coupling. Seeing the robustness of similar VPT2 results across different methods for the methanol dimer and methanol-ethene, it is possible that these results are representative of those that would be obtained at higher electronic structure methods.

Concerning the $\mathrm{MA}_{\pi}$ conformer, the harmonic calculations suggest the $\mathrm{OH} \cdots \pi$ hydrogen bond to be comparably strong as in methanol-ethene, whereas the observable $55 \mathrm{~cm}^{-1}$ dimerization shift would suggest a slightly stronger binding. The discrepancy may either stem from a deficiency in the calculations in judging the energetics of the contact, or from additional anharmonic effects which are not taken into account here. Without an observation of the corresponding $\mathrm{MA}_{\pi}$ overtone band however, it will be difficult to judge the balance between diagonal and off-diagonal anharmonic contributions in this system. Boosting the relative amount of $\mathrm{OH} \cdots \pi$-bonded structures through appropriate ring substitution might be helpful in this regard if vapor-pressure limitations do not come into effect. ${ }^{147}$

Based on the SCS-LMP2 results, an attempt at explaining the absolute $\mathrm{MA}_{O}$ dimer stretching wavenumber of $\tilde{v}_{\mathrm{S}}^{\text {Dim }}=3598 \mathrm{~cm}^{-1}$ can be made. Combining the $\omega_{\mathrm{s}}^{\text {Dim }}=3774 \mathrm{~cm}^{-1}$ harmonic wavenumber from Table 3.5 with the experimentally determined $x_{\mathrm{s}, \mathrm{s}}^{\mathrm{Dim}}=-96 \mathrm{~cm}^{-1}$ diagonal anharmonicity content predicts an overall $+18 \mathrm{~cm}^{-1}$ blue shift due to the halved off-diagonal anharmonic sum, $\frac{1}{2} \sum x_{\mathrm{s}, i}^{\mathrm{Dim}}$. Since the choice of SCS wavenumbers as the starting point for this analysis is only based on an empirical rule from the two model systems studied above, a higher-level confirmation of these results would be necessary to verify this approach. Still, the overall blue-shifting direction is qualitatively consistent with the methanol dimer (where it amounts to $+33 \mathrm{~cm}^{-1}$ ), and would further increase in magnitude if one approached the unscaled LMP2 wavenumbers. It thus appears plausible to assume similarly blue-shifting off-diagonal corrections for other $\mathrm{OH} \cdots \mathrm{O}$ hydrogen bonds, two examples of which will be investigated below.

\subsection{Related OH $\cdots$ O Hydrogen Bonds in Aliphatic Alcohols}

With the thorough analysis of the methanol dimer in terms of its diagonal $\mathrm{OH}$ stretching anharmonicity and harmonic estimates in Chapter 2, it is 

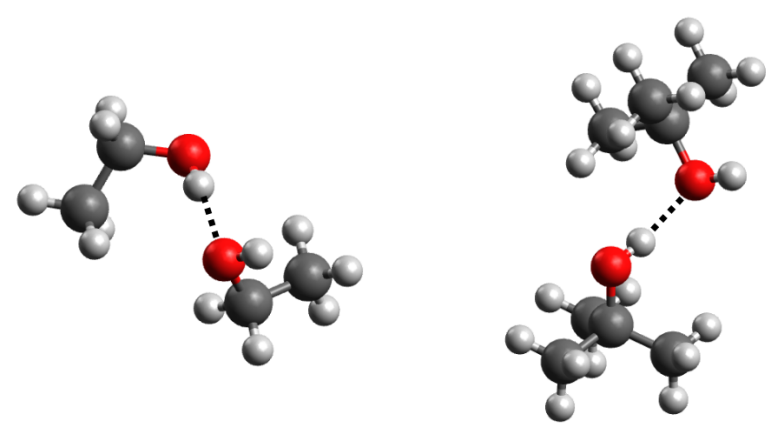

Figure 3.6: Structures of the most stable homodimers of ethanol (gghom structure, left) and tert-butyl alcohol (right).

desirable to see whether the findings for this $\mathrm{OH} \cdots \mathrm{O}$ contact qualitatively uphold in other homodimers of aliphatic alcohols. From the overtone study by Kollipost et al. in Reference 14, diagonal $\mathrm{OH}$ anharmonicity data is available for the homodimers of ethanol and tert-butyl alcohol. Based on the adequate agreement of harmonic SCS-LMP2 predictions with the Coupled Cluster benchmarks for methanol monomers and dimers (Section 2.3), similar calculations were conducted for these two systems. The data are assembled in Table 3.6.

For ethanol, calculations similar to the "LMOMO+C-O(int)" scheme in methanol (Section 2.3) were also carried out. Again, this scheme includes the electron pairs in the $\mathrm{C}-\mathrm{O}$ and $\mathrm{O}-\mathrm{H}$ bonds, the oxygen lone pairs, and all intermolecular excitations at the $\operatorname{CCSD}(\mathrm{T})$ level.

While tert-butyl alcohol is monoconformational, the OH-torsional isomerism in ethanol demands a careful matching of the assigned spectral transitions to the possible monomer and dimer structures. ${ }^{104}$ The preferred trans conformation in the monomer is converted to a homochiral doublegauche conformation in the most stable dimer structure (depicted in Figure 3.6). Focusing on the trans monomer reference would be advantageous in that its $\mathrm{OH}$ stretching band is free of tunneling splittings, but might disturb the picture when going to the dimer due to the switch in conformational preference. Thus, the gauche monomer is chosen as the reference throughout. 
Table 3.6: Assembled spectroscopic data (in $\mathrm{cm}^{-1}$ ) for the monomers and homodimers of methanol (Sec. 2), ethanol (gauche monomer/gg hom dimer), and tert-butyl alcohol, from Ref. 14, including negative sums $(-\Delta)$ pertaining to dimerization shifts. The full off-diagonal sums are calculated by closing the gap to the experiment, based on the respective harmonic predictions printed in italics.

\begin{tabular}{|c|c|c|c|c|c|c|c|c|c|}
\hline & \multicolumn{3}{|c|}{$\mathrm{MeOH}$} & \multicolumn{3}{|c|}{ EtOH $\left(g g_{\text {hom }}\right)$} & \multicolumn{3}{|c|}{$t-\mathrm{BuOH}$} \\
\hline & Mon & Dim & $-\Delta$ & Mon & Dim & $-\Delta$ & Mon & Dim & $-\Delta$ \\
\hline \multicolumn{10}{|l|}{$\omega_{\mathrm{s}}:$} \\
\hline LMP2 $^{\text {a }}$ & 3857 & 3709 & 148 & 3833 & 3677 & 156 & 3826 & 3682 & 145 \\
\hline SCS-LMP2 ${ }^{\mathrm{a}}$ & 3852 & 3740 & 113 & 3832 & 3713 & 119 & 3829 & 3722 & 107 \\
\hline LMOMO $^{b}$ & 3836 & 3730 & 106 & 3813 & 3699 & 114 & & & \\
\hline Best est. ${ }^{c}$ & 3862 & 3740 & 121 & 3840 & & & & & \\
\hline$\tilde{v}_{\mathrm{S}}(\exp )$. & 3684 & 3575 & 109 & 3659 & 3532 & 128 & 3642 & 3497 & 145 \\
\hline$(2 \tilde{v})_{\mathrm{S}}($ exp. $)$ & 7196 & 6951 & 245 & 7144 & 6861 & 283 & 7111 & 6789 & 322 \\
\hline $2 x_{\mathrm{s}, \mathrm{s}}$ & -171 & -198 & +27 & -176 & -202 & +26 & -174 & -205 & +31 \\
\hline$\frac{1}{2} \sum x_{\mathrm{s}, i}$ & -7 & +33 & -40 & -5 & +20 & -25 & -13 & -20 & +7 \\
\hline
\end{tabular}

\subsubsection{Ethanol}

The gauche monomer of ethanol displays tunneling interactions between its two enantiomeric forms, leading to a subtle $3.3 \mathrm{~cm}^{-1}$ tunneling splitting of its vibrational states ${ }^{37}$ which would in principle warrant similar localization considerations as in the methanol monomer. Kollipost et al. ${ }^{14}$ have discussed a number of plausible assignments to the transitions between the different tunneling states in their overtone spectra, with moderate remaining uncertainties. Their findings suggest fundamental and overtone transition band centers around $\tilde{v}_{\mathrm{s}}^{\text {Mon }}=3659(1) \mathrm{cm}^{-1}$ and $(2 \tilde{v})_{\mathrm{s}}^{\text {Mon }}=7144(2) \mathrm{cm}^{-1}$, and a diagonal anharmonicity constant of $x_{\mathrm{s}, \mathrm{s}}^{\mathrm{Mon}}=-88(2) \mathrm{cm}^{-1}$. For the dimer, the tunneling ambiguities are again lifted due to the lateral confinement of the $\mathrm{OH}$ group in the hydrogen bond, and the corresponding gghom transitions at $\tilde{v}_{\mathrm{s}}^{\text {Dim }}=3532 \mathrm{~cm}^{-1}$ and $(2 \tilde{v})_{\mathrm{s}}^{\text {Dim }}=6861 \mathrm{~cm}^{-1}$ yield a diagonal anharmonicity constant of $x_{\mathrm{s}, \mathrm{s}}^{\text {Dim }}=-101 \mathrm{~cm}^{-1}$.

The size of the ethanol monomer still allows for full $\operatorname{LCCSD}\left(\mathrm{T} 0^{*}\right)$ - 
F12a/VDZ-F12 treatments, which yield a best harmonic value for the free $\mathrm{OH}$ stretching wavenumber of $3840 \mathrm{~cm}^{-1}$ (see Table 3.6). LMP2 and SCSLMP2 are both within $10 \mathrm{~cm}^{-1}$ of this value, while the "LMOMO+C-O" scheme-simulating a full LCCSD(T0) treatment—falls about $30 \mathrm{~cm}^{-1}$ short of it. Again, SCS appears to be beneficial for the harmonic dimerization shift, seeing that it is again considerably closer to the Coupled Cluster value than the standard LMP2 estimate.

For the dimer, the "LMOMO+C-O" results represent the highest available level of theory, predicting a harmonic dimerization shift of $114 \mathrm{~cm}^{-1}$. If similar patterns as for the methanol dimer and methanol-ethene uphold, this method would tend to underestimate the dimerization shift, and one can assume the results in Table 3.6 to bracket any higher-level references. At this point, it is again instructive to investigate the absolute $\mathrm{OH}$ stretching wavenumber in the dimer in addition to the dimerization shift. Using the SCS-LMP2 results as a reference implies an off-diagonal correction $\frac{1}{2} \sum x_{\mathrm{s}, i}^{\mathrm{Dim}}$ of $+20 \mathrm{~cm}^{-1}$, while the LMOMO result qualitatively agrees on the blueshifting direction of this correction. This is compatible with the methanol homodimer and methanol-anisole $\mathrm{OH} \cdots \mathrm{O}$ situations, where it amounts to +33 and $+18 \mathrm{~cm}^{-1}$, respectively.

Overall, the experiments suggest a slightly stronger $\mathrm{OH} \cdots \mathrm{O}$ hydrogen bond than in the methanol dimer, as evidenced by the larger dimerization shift and the more pronounced overtone intensity attenuation by a factor of 400(100). ${ }^{14}$ The calculations follow this finding in terms of the harmonic dimerization shift, although the tentative anharmonic analysis presented above is less certain in this regard due to the lack of robust harmonic benchmarks.

\subsection{2 tert-Butyl Alcohol}

The analysis of the third experimental data set for the tert-butyl alcohol homodimer, depicted in Figure 3.6, is hampered on the quantum chemical side by the large size of the system. Only LMP2 and SCS-LMP2/sVTZ calculations were feasible in this case, lacking the partial LMOMO inclusion of Coupled Cluster correlation that was still possible in ethanol. The data included in Table 3.6 thus carry large uncertainties, and one can only analyze them in terms of their qualitative compatibility to the cases discussed above.

The absence of significant tunneling effects in the monomer $\mathrm{OH}$ stretching band relieves the need for a localization analysis. ${ }^{148}$ From the unambiguous fundamental and overtone band assignments, Kollipost et al. have arrived at a diagonal $\mathrm{OH}$ stretching anharmonicity of $x_{\mathrm{s}, \mathrm{s}}=-88 \mathrm{~cm}^{-1}$ in the 
monomer, and $-103 \mathrm{~cm}^{-1}$ in the dimer. The observable dimerization shift in the fundamental region amounts to $145 \mathrm{~cm}^{-1}$, suggesting a further gain in hydrogen bond strength over the ethanol case, which is supported by the 1000(400)-fold overtone intensity attenuation.

Again, SCS-LMP2 predicts a much smaller harmonic dimerization shift than LMP2. In contrast to all previous systems however, the unscaled LMP2/sVTZ estimates now coincide with the experimental dimerization shift instead of significantly overshooting it. Based on these results, one would arrive at a blue-shifting off-diagonal contribution of $\frac{1}{2} \sum_{\mathrm{s}, i}^{\mathrm{Dim}}=$ $-20 \mathrm{~cm}^{-1}$, negating the change in diagonal anharmonicity. Conversely, using the much smaller SCS-LMP2 dimerization shift implies that the off-diagonal shift is slightly red-shifting (as demonstrated in Table 3.6), unlike all other $\mathrm{OH}$... O cases discussed before. Without higher-level harmonic references, it must remain undecided whether this discrepancy arises from misjudged harmonic shifts, or a true effect in the summed off-diagonal contributions.

\subsection{Summary}

In this chapter, the $\mathrm{OH} \cdots \pi$ hydrogen bond in the methanol-ethene complex has been studied in analogy to the methanol homodimer in Chapter 2. The small observable dimerization shift $\left(-\Delta \tilde{v}_{\mathrm{S}}=43 \mathrm{~cm}^{-1}\right)$ is almost fully explained by the best harmonic predictions $\left(-\Delta \omega_{\mathrm{s}}=45 \mathrm{~cm}^{-1}\right)$, and the minute change in diagonal anharmonicity $\left(-2 \Delta x_{\mathrm{S}, \mathrm{S}}=6 \mathrm{~cm}^{-1}\right)$ implies only moderate off-diagonal corrections $\left(\frac{1}{2} \sum \Delta x_{\mathrm{s}, i}=-8 \mathrm{~cm}^{-1}\right)$. This is corroborated by a moderate stretching overtone attenuation factor $(170 \pm 70)$, a low predicted dissociation energy $\left(D_{0}=7.7 \mathrm{~kJ} \mathrm{~mol}^{-1}\right)$, and a VPT2 estimate for the stretchinglibration coupling constant of only $x_{\mathrm{s}, 1}^{\mathrm{Dim}}=+16 \mathrm{~cm}^{-1}$. Overall, the data indicate a rather weak hydrogen bond, and clearly contrast the much stronger $\mathrm{OH} \cdots \mathrm{O}$ contact in the methanol dimer.

Based on these two data sets, the two competing interaction motifs in methanol-anisole clusters were assigned in jet-FTIR spectra, indicating a clear preference for $\mathrm{OH} \cdots \mathrm{O}$ over $\mathrm{OH} \cdots \pi$ binding ${ }^{52}$ The results thereby provide a vital benchmark for quantum chemical calculations, where many popular methods tend to predict a close energetic match between both binding options. This problem was originally alleviated by including Coupled Cluster corrections, but switching to a local-correlation picture also appears to be beneficial at the MP2 level.

Since the methanol dimer represents the simplest aliphatic $\mathrm{OH} \cdots \mathrm{O}$ hydrogen bond, it is instructive to compare its vibrational dynamics to those found in other alcohols. To this end, data on ethanol and tert-butyl alco- 
hol from an earlier overtone study ${ }^{14}$ were subjected to a tentative analysis based on a set of new harmonic quantum chemical calculations. The size of these systems starts to limit the applicability of more accurate computational approaches, but Coupled Cluster contributions were successfully included in the ethanol dimer for the interacting $\mathrm{OH}$ groups. Furthermore, the methanol homodimer and methanol-ethene calculations suggest an empirical benefit of Spin-Component Scaling for harmonic predictions in light of high-level benchmarks. Deriving some tentative anharmonic data for methanol-anisole and ethanol dimers by similar means provides a qualitatively consistent picture, but the extendibility to the tert-butyl alcohol case is somewhat unclear. In any case, the role of SCS predictions remains speculative unless more accurate calculations provide a reference for these systems.

Finally, the experimental findings for the alcohol dimers seem to indicate that it is difficult to quantify a correlation between the overall observable dimerization shift, the increase in diagonal anharmonicity, and the magnitude of the stretching-libration coupling. The steady increase of the dimerization shift from methanol $\left(109 \mathrm{~cm}^{-1}\right)$ to ethanol $\left(128 \mathrm{~cm}^{-1}\right)$ and tert-butyl alcohol $\left(145 \mathrm{~cm}^{-1}\right)$ is accompanied by an only slight increase in diagonal anharmonicity content $\left(13,13\right.$ and $16 \mathrm{~cm}^{-1}$ respectively). In addition, an exploratory anharmonic calculation for the ethanol dimer predicts a stretching-libration coupling $\left(+55 \mathrm{~cm}^{-1}\right)$ on par with the prediction for methanol $\left(+58 \mathrm{~cm}^{-1}\right)$. Although VPT2 predictions have been found to misjudge this quantity in the methanol dimer, they have also demonstrated a distinct robustness among different methods. This raises the question whether predictions for the ethanol dimer at other computational levels would fall in line with this closeness to the methanol case; and furthermore, if the results are likewise in systematic disagreement with the true values. It would thus be desirable to expend more dedicated theoretical and experimental efforts in order to explain the anharmonic situation in this system for which higher-level calculations, possibly with advanced explicitly correlated LMOMO implementations, would still be within reach. Gathering similar experimental and theoretical data for isopropyl alcohol dimers could bridge the remaining gap between the ethanol and tert-butyl alcohol data and complete this series on the evolution of spectroscopic parameters with the number of attached methyl groups. 


\section{Summary}

The impact of anharmonicity on the vibrational dynamics in hydrogenbonded systems has been investigated in this work on several examples. The largest efforts have been spent on elucidating the situation in the methanol homodimer as a model for $\mathrm{OH} \cdots \mathrm{O}$ hydrogen bonding, and methanol-ethene as a $\mathrm{OH} \cdots \pi$ prototype. Based on the extensive tunneling interactions in the methanol monomer, localization procedures have been put forward in order to reduce the intricate experimental situation to a form which can be compared to quantum chemical predictions and provide a less ambiguous reference to define spectroscopic dimerization shifts.

Combining a selection of experimental and theoretical data on the two model systems, one can conclude that their donor $\mathrm{OH}$ stretching wavenumbers encode an overall blue-shifting contribution from the important stretching-libration coupling constant, which has also been determined experimentally for the methanol homodimer. Furthermore, it was found that popular DFT and MP2 calculations tend to exaggerate harmonic dimerization shifts, and that applying Spin-Component Scaling is beneficial in bringing the harmonic results into closer agreement with high-level benchmarks.

A number of additional spectroscopic results on methanol-anisole, ethanol, and tert-butyl alcohol dimers were subjected to similar analyses. While the ethanol dimer still shows the same qualitative patterns as in the methanol case, the tert-butyl alcohol appears to fall out of line either in the harmonic predictions or the resulting off-diagonal anharmonic corrections. However, the feasibility of high-level quantum chemical methods starts to break down for these systems. Any conclusions that one can attempt to draw from comparison with more well-studied cases therefore remains speculative until improved harmonic references are available.

With the data gathered from these systems, one can critically assess the widespread approach of scaling harmonic predictions in order to approximate anharmonic band positions. As Nibbering has stated in a 2007 review, ${ }^{9}$ "[w]eak hydrogen bonds with $\mathrm{X}-\mathrm{H}$ stretching frequencies above $3200 \mathrm{~cm}^{-1}$ can often be sufficiently described in the harmonic approximation applying 
an empirical scaling factor". The data gathered throughout this work highlight the drawbacks of such simple approaches: the overall band positions are the result of counteracting diagonal and off-diagonal anharmonic corrections, and the large number of normal modes contained in the latter makes it difficult to estimate their overall effect. In addition, the results across the methanol, ethanol, and tert-butyl alcohol dimers in Section 3.3 do not appear to show a clear correlation between the most important contributions to the dimerization shift.

The aforementioned review continues in stating that "[a] onedimensional anharmonic correction in the proton coordinate" should suffice beyond the simple scaling approach in order to estimate anharmonic band positions. ${ }^{9}$ Such calculations have been carried out for the methanol dimer in Section 2.3.1 and methanol-ethene in Section 3.1.2 at a high level of theory, revealing that the transitions obtained for the methanol dimer distinctly differ from the experimental ones due to the neglect of important off-diagonal anharmonic effects. Only in the methanol-ethene case did this approach provide helpful, but this system clearly represents an extreme case due to the very weak hydrogen bond, for which the agreement of the 1-D anharmonic values with the experiment appears to be serendipitous. From the data presented in this work, one can actually assume robust harmonic estimates to be a generally better estimate of true band positions than such one-dimensional anharmonic results. Obtaining such estimates does however require computational efforts and advanced methods which are still often unfeasible and not widely available today.

One can conclude that the experimental effort to obtain anharmonicity information for hydrogen-bonded systems is generally prohibitive for routine applications due to unfavorable intensity effects and the need for accurate band assignments. It remains to be seen to what extent improved theoretical methods are able to provide accurate harmonic and anharmonic predictions for medium-sized to large molecular systems in the future; hopefully, the findings presented in this work can provide a point of reference for upcoming developments and applications. 


\section{Appendix A}

\section{Experimental Parameters}

Given below are the experimental parameters for the spectra shown in this work: Dates (YY/MM/DD) and identification letters, where available; detectors and preamplifier settings ("Det." and "Preamp"); light sources; filters; optics materials; apertures ("Apt.", in $\mathrm{mm}$ ); scanner velocities ("Scanner", internal setting in $\mathrm{kHz}$ ); spectral resolution ("Res.", in $\mathrm{cm}^{-1}$ ); number of coadded scans or scanning times ("Scans", times indicates by seconds); and details on the samples.

For the jet measurements, three different detectors were used: the newer one of two available InSb/MCT sandwich detectors ("InSb SW"); and two InGaAs detectors ("InGaAs old" and "new"). As outlined in Section 1.2.2, both 50 and $150 \mathrm{~W}$ tungsten lamps were used as light sources ("W50" and "W150"). The specified optics materials were used for all lenses, windows, and the beamsplitter. The sample details include the temperatures and helium pressures at which the samples were stored in the saturators (in the form “Temperature@Pressure"). Ethene samples were pre-mixed with helium in a gas cylinder at the specified volume ratios. Also given are the synchronization times (on/off) of the reservoir feeding valves ("cont." = continuous feeding), and the stagnation pressure $p_{\mathrm{s}}$ in the reservoir.

For the matrix spectra (Figure 2.7), deposition was carried out from a 10 mbar methanol reservoir through a partially opened needle valve, together with a total of 60 mbar of neon from a different reservoir. For the older libration fundamental spectrum from Kollipost et al. ${ }^{103}$ sample deposition was carried out from a 3.9 mbar $\mathrm{MeOH}$ reservoir and a total of $50 \mathrm{mbar}$ Ne. For all matrix spectra, the optics materials refer only to the beamsplitter. 
Table A.1: Measurement parameters for NOTCH curves (Figure 1.2).

\begin{tabular}{lcccccccccc}
\hline & Date/ID & Detector & Preamp. & Source & Filter & Optics & Apt. & Scanner & Res. & Scans \\
\hline "InSb" & $15 / 11 / 09, .1$ & InSb SW & $2.4 \mathrm{k} \Omega$ & $\mathrm{W} 50$ & $13 \mathrm{a}$ & $\mathrm{CaF}_{2}$ & 3.5 & 80 & 2 & $60.15 \mathrm{~s}$ \\
& $15 / 09 / 30, .0$ & $\mathrm{InSb} \mathrm{SW}$ & $2.7 \mathrm{k} \Omega$ & $\mathrm{W} 50$ & $13 \mathrm{a}$ & $\mathrm{CaF}_{2}$ & 3.5 & 80 & 2 & $60.15 \mathrm{~s}$ \\
& $15 / 11 / 09, .2$ & $\mathrm{InSb} \mathrm{SW}$ & $2.4 \mathrm{k} \Omega$ & $\mathrm{W} 150$ & $13 \mathrm{a}$ & $\mathrm{CaF}_{2}$ & 3.5 & 80 & 2 & $60.15 \mathrm{~s}$ \\
& $15 / 09 / 30, .0$ & $\mathrm{InSb} \mathrm{SW}$ & $2.7 \mathrm{k} \Omega$ & $\mathrm{W} 150$ & $13 \mathrm{a}$ & $\mathrm{CaF}_{2}$ & 3.5 & 80 & 2 & $60.15 \mathrm{~s}$ \\
"InGaAs" & $15 / 11 / 09, .2$ & InGaAs (new) & $2.0 \mathrm{k} \Omega, 1.0 \mathrm{nF}$ & $\mathrm{W} 50$ & 16 & $\mathrm{CaF}_{2}$ & 4.0 & 80 & 2 & $60.15 \mathrm{~s}$ \\
& $15 / 09 / 30, .1$ & InGaAs (new) & $2.0 \mathrm{k} \Omega, 1.0 \mathrm{nF}$ & $\mathrm{W} 50$ & 16 & $\mathrm{CaF}_{2}$ & 4.0 & 80 & 2 & $60.15 \mathrm{~s}$ \\
& $15 / 11 / 09, .2$ & InGaAs (new) & $2.0 \mathrm{k} \Omega, 1.0 \mathrm{nF}$ & $\mathrm{W} 150$ & 16 & $\mathrm{CaF}_{2}$ & 4.0 & 80 & 2 & $60.15 \mathrm{~s}$ \\
& $15 / 09 / 30, .0$ & InGaAs (new) & $2.0 \mathrm{k} \Omega, 1.0 \mathrm{nF}$ & $\mathrm{W} 150$ & 16 & $\mathrm{CaF}_{2}$ & 4.0 & 80 & 2 & $60.15 \mathrm{~s}$ \\
\hline
\end{tabular}

Table A.2: Measurement parameters for methanol jet-FTIR spectra (Figure 2.6).

\begin{tabular}{|c|c|c|c|c|c|c|c|c|c|c|c|}
\hline & Date/ID & Detector & Preamp. & Source & Filter & Optics & Apt. & Scanner & Res. & Scans & Sample \\
\hline \multicolumn{12}{|c|}{ Top (overtone region) } \\
\hline (Ref. 14) & $12 / 08 / 13$ & InGaAs (old) & $3.1 \mathrm{k} \Omega, 560 \mathrm{pF}$ & W50 & 16 & $\mathrm{CaF}_{2}$ & 4.0 & 60 & 2 & 1450 & $\begin{array}{l}\mathrm{MeOH}:-13^{\circ} \mathrm{C} @ 1.6 \text { bar, cont.; } \\
\mathrm{He} @ 1.6 \text { bar, cont.; } \\
p_{\mathrm{s}}=0.8 \text { bar }\end{array}$ \\
\hline \multicolumn{12}{|c|}{ Bottom (fundamental region) } \\
\hline & $14 / 09 / 19, \mathrm{~h}$ & InSb SW & $2.7 \mathrm{k} \Omega$ & W150 & $13 \mathrm{a}$ & $\mathrm{CaF}_{2}$ & 3.5 & 80 & 2 & 100 & $\begin{array}{l}\mathrm{MeOH}:-25^{\circ} \mathrm{C} @ 1.6 \mathrm{bar}, 0.80 / 0.20 \mathrm{~s} ; \\
\mathrm{He} @ 1.6 \mathrm{bar}, 0.20 / 0.80 \mathrm{~s} ; \\
p_{\mathrm{s}}=0.35 \mathrm{bar}\end{array}$ \\
\hline
\end{tabular}


Table A.3: Measurement parameters for methanol matrix-FTIR spectra (Figure 2.7).

\begin{tabular}{|c|c|c|c|c|c|c|c|c|c|}
\hline & Date/ID & Detector & Source & Optics & Apt. & Scanner & Res. & Scans & Sample \\
\hline \multicolumn{10}{|c|}{ "OH stretching overtone", "OH stretching fundamental”, "OH stretching-libration combination" } \\
\hline & $15 / 06 / 09$ & $\mathrm{InSb}$ & Tungsten & $\mathrm{CaF}_{2}$ & 8.0 & 40 & 0.5 & $1750 \mathrm{~s}$ & $\begin{array}{l}\mathrm{MeOH}: 10 \text { mbar reservoir; } \\
60 \text { mbar total Ne deposition }\end{array}$ \\
\hline \multicolumn{10}{|c|}{ “OH libration overtone" } \\
\hline & 15/06/09 & MCT & Globar & $\mathrm{KBr}$ & 4.0 & 40 & 0.5 & $874 \mathrm{~s}$ & $\begin{array}{l}\mathrm{MeOH}: 10 \text { mbar reservoir; } \\
60 \text { mbar total Ne deposition }\end{array}$ \\
\hline \multicolumn{10}{|c|}{ "OH libration fundamental" } \\
\hline (Ref. 103) & $12 / 11 / 09$ & Bolometer & Globar & Mylar, $6 \mu \mathrm{m}$ & 12.5 & 10 & 1 & $800 \mathrm{~s}$ & $\begin{array}{l}\mathrm{MeOH}: 3.9 \text { mbar reservoir; } \\
50 \text { mbar total Ne deposition }\end{array}$ \\
\hline
\end{tabular}

Table A.4: Measurement parameters for methanol-ethene spectra (Figure 3.2, top).

\begin{tabular}{|c|c|c|c|c|c|c|c|c|c|c|c|}
\hline & Date/ID & Detector & Preamp. & Source & Filter & Optics & Apt. & Scanner & Res. & Scans & Sample \\
\hline \multicolumn{12}{|c|}{ Top (overtone region) } \\
\hline Strong trace & $\begin{array}{l}14 / 04 / 11- \\
14 / 04 / 15\end{array}$ & InGaAs (new) & $2.0 \mathrm{k} \Omega, 1.0 \mathrm{nF}$ & W150 & 16 & $\mathrm{CaF}_{2}$ & 4.0 & 80 & 2 & 1200 & $\begin{array}{l}\mathrm{MeOH}:-15^{\circ} \mathrm{C} @ 1.6 \mathrm{bar} \text {, cont.; } \\
\text { Ethene: } 10 \% \text { in He@1.8 bar, cont.; } \\
p_{\mathrm{s}}=0.75 \mathrm{bar}\end{array}$ \\
\hline Thin black tr. & $\begin{array}{l}14 / 06 / 04- \\
14 / 07 / 10\end{array}$ & InGaAs (new) & $2.0 \mathrm{k} \Omega, 1.0 \mathrm{nF}$ & W150 & 16 & $\mathrm{CaF}_{2}$ & 4.0 & 80 & 2 & 1050 & $\begin{array}{l}\text { Ethene: } 10 \% \text { in } \mathrm{He} @ 1.8 \text { bar, cont.; } \\
\text { He@1.6 bar, cont.; } \\
p_{\mathrm{s}}=0.75 \text { bar }\end{array}$ \\
\hline Grey trace & \multicolumn{11}{|c|}{ methanol overtone spectrum, as in Fig. 2.6} \\
\hline \multicolumn{12}{|c|}{ Bottom (fundamental region) } \\
\hline Black trace & $\begin{array}{l}14 / 01 / 31, \mathrm{~b} \\
02 / 06 / 14, \mathrm{c}\end{array}$ & InSb SW & $2.7 \mathrm{k} \Omega$ & $\mathrm{W} 150$ & $13 \mathrm{a}$ & $\mathrm{CaF}_{2}$ & 3.5 & 80 & 2 & 150 & $\begin{array}{l}\mathrm{MeOH}:-25^{\circ} \mathrm{C} @ 1.6 \mathrm{bar}, 0.10 / 1.11 \mathrm{~s} \text {; } \\
\text { Ethene: } 2 \% \text { in He@1.8 bar, } 0.95 / 0.94 \mathrm{~s} \text {; } \\
p_{\mathrm{s}}=0.75 \mathrm{bar}\end{array}$ \\
\hline Grey trace & $\begin{array}{l}14 / 06 / 17- \\
14 / 06 / 19\end{array}$ & InSb SW & $3.1 \mathrm{k} \Omega$ & $\mathrm{W} 150$ & $13 \mathrm{a}$ & $\mathrm{CaF}_{2}$ & 3.5 & 80 & 2 & 170 & $\begin{array}{l}\text { MeOH: }-15^{\circ} \mathrm{C} @ 1.6 \text { bar, cont.; } \\
\text { Ethene: } 10 \% \text { in He@1.8 bar, cont.; } \\
p_{\mathrm{s}}=0.75 \mathrm{bar}\end{array}$ \\
\hline
\end{tabular}


Table A.5: Measurement parameters for methanol-ethene concentration series (Figure 3.2, bottom).

\begin{tabular}{|c|c|c|c|c|c|c|c|c|c|c|c|}
\hline & Date/ID & Detector & Preamp. & Source & Filter & Optics & Apt. & Scanner & Res. & Scans & Sample \\
\hline Black trace & \multicolumn{11}{|c|}{ as in fundamental spectrum (top part of Fig. 3.2, lower panel) } \\
\hline$" \times 0.79 "$ & $14 / 06 / 03, a+e$ & InSb SW & $2.7 \mathrm{k} \Omega$ & W150 & $13 \mathrm{a}$ & $\mathrm{CaF}_{2}$ & 3.5 & 80 & 2 & 100 & $\begin{array}{l}\mathrm{MeOH}:-25^{\circ} \mathrm{C} @ 1.6 \mathrm{bar}, 0.56 / 1.13 \mathrm{~s} \text {; } \\
\text { Ethene: } 2 \% \text { in He@1.8 bar, } 0.95 / 0.94 \mathrm{~s} ; \\
p_{\mathrm{s}}=0.75 \mathrm{bar}\end{array}$ \\
\hline “×0.81" & $14 / 06 / 03, b+d$ & InSb SW & $2.7 \mathrm{k} \Omega$ & W150 & $13 \mathrm{a}$ & $\mathrm{CaF}_{2}$ & 3.5 & 80 & 2 & 100 & $\begin{array}{l}\text { MeOH: }-25^{\circ} \mathrm{C} @ 1.6 \text { bar, } 1.00 / 1.13 \mathrm{~s} \text {; } \\
\text { Ethene: } 2 \% \text { in He@1.8 bar, } 0.54 / 0.94 \mathrm{~s} ; \\
p_{\mathrm{s}}=0.75 \text { bar }\end{array}$ \\
\hline$" \times 1.5 "$ & $14 / 06 / 03, \mathrm{c}$ & InSb SW & $2.7 \mathrm{k} \Omega$ & W150 & $13 a$ & $\mathrm{CaF}_{2}$ & 3.5 & 80 & 2 & 100 & $\begin{array}{l}\text { MeOH: }-25^{\circ} \mathrm{C} @ 1.6 \mathrm{bar}, 1.00 / 1.13 \mathrm{~s} \text {; } \\
\text { Ethene: } 2 \% \text { in } \mathrm{He} @ 1.8 \text { bar, } 0.10 / 0.94 \mathrm{~s} ; \\
\mathrm{He} @ 1.6 \text { bar, } 0.49 / 0.48 \mathrm{~s} ; \\
p_{\mathrm{S}}=0.75 \mathrm{bar}\end{array}$ \\
\hline
\end{tabular}

Table A.6: Measurement parameters for methanol-anisole spectra (Figure 3.5).

\begin{tabular}{|c|c|c|c|c|c|c|c|c|c|c|c|}
\hline & Date/ID & Detector & Preamp. & Source & Filter & Optics & Apt. & Scanner & Res. & Scans & Sample \\
\hline \multicolumn{12}{|c|}{ Top (OH overtone region) } \\
\hline Black trace & $\begin{array}{l}14 / 11 / 25, a+b \\
14 / 11 / 26, a+b\end{array}$ & InGaAs (new) & $2.0 \mathrm{k} \Omega, 1.0 \mathrm{nF}$ & W150 & 16 & $\mathrm{CaF}_{2}$ & 4.0 & 80 & 2 & 1200 & $\begin{array}{l}\mathrm{MeOH}:-20^{\circ} \mathrm{C} @ 1.6 \mathrm{bar}, 0.51 / 0.55 \mathrm{~s} \text {; } \\
\text { Anisole: } 20^{\circ} \mathrm{C} @ 1.8 \text { bar, } 0.91 / 0.10 \mathrm{~s} ; \\
p_{\mathrm{s}}=0.75 \text { bar }\end{array}$ \\
\hline Grey trace & \multicolumn{11}{|c|}{ methanol overtone spectrum, as in Fig. 2.6} \\
\hline \multicolumn{12}{|c|}{ Middle (OH fundamental region) } \\
\hline Black trace & $\begin{array}{l}\text { 14/12/09, a; } \\
15 / 01 / 16, \text { a-c; } \\
15 / 01 / 19, \text { c-g+k }\end{array}$ & $\mathrm{InSb} \mathrm{SW}$ & $2.7 \mathrm{k} \Omega$ & $\mathrm{W} 150$ & $13 \mathrm{a}$ & $\mathrm{CaF}_{2}$ & 3.5 & 80 & 2 & 210 & $\begin{array}{l}\text { MeOH: }-20^{\circ} \mathrm{C} @ 1.6 \mathrm{bar}, 0.51 / 0.55 \mathrm{~s} \text {; } \\
\text { Anisole: } 20^{\circ} \mathrm{C} @ 1.8 \mathrm{bar}, 0.91 / 0.10 \mathrm{~s} \text {; } \\
p_{\mathrm{s}}=0.75 \mathrm{bar}\end{array}$ \\
\hline Grey trace & methanol fundar & ntal spectrum, & in Fig. 2.6 & & & & & & & & \\
\hline \multicolumn{12}{|c|}{ Bottom (OD fundamental region) } \\
\hline & $15 / 02 / 03$, a & InSb SW & $2.7 \mathrm{k} \Omega$ & W150 & $13 \mathrm{a}$ & $\mathrm{CaF}_{2}$ & 3.5 & 80 & 1.5 & 150 & $\begin{array}{l}\text { MeOD: }-20^{\circ} \mathrm{C} @ 1.6 \text { bar, } 0.52 / 0.55 \mathrm{~s} \text {; } \\
\text { Anisole: } 20^{\circ} \mathrm{C} @ 1.8 \text { bar, } 0.94 / 0.10 \mathrm{~s} ; \\
p_{\mathrm{S}}=0.75 \mathrm{bar}\end{array}$ \\
\hline
\end{tabular}




\section{Appendix B}

\section{Numeric MatLAB Routines}

\section{B.1 Outline and Source Code Excerpts}

A set of custom MATLAB routines was written to calculate numerical timeindependent solutions to the Schrödinger equation of the rotational/vibrational problems presented in this work. Below, a basic description of the program flow is provided, and central code sections are reproduced. The given code examples include the following physical constants in their respective SI units: $h$ and hbar as Planck's (reduced) constant; c as the speed of light in vacuum; and NA as Avogadro's constant. Further, the following unit conversions are used: kgtou from kilograms to atomic mass units; Jtocm from Joules to reciprocal centimeters; and mtoA from meters to Ångstrom. Built-in MATLAB commands and functions are capitalized throughout.

First, the displacement coordinate interval $\mathrm{x}$ is mapped out by a regular grid of sufficiently fine resolution Dx. Along this coordinate, a basis set is constructed by first defining a single "seeding" function, user-specified as the ground-state harmonic vibrational wavefunction $(v=0)$ of an oscillator of a given mass $\mu$ (mu, in $\mathrm{u}$ ) and wavenumber $\omega$ (omega, in $\left.\mathrm{cm}^{-1}\right)$. Drawing from the formulation chosen by Hollas in Reference $21^{*}$, this first wavefunction psi is defined by:

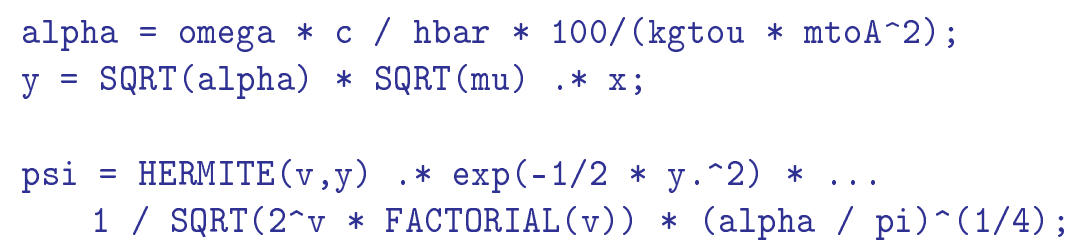

\footnotetext{
*Note that in comparison to Eqn. (1.70) in Ref. 21, the formula used herein includes an additional factor of $\alpha^{1 / 4}$ in the normalization constant. This is necessary due to subsequent integration over the Cartesian coordinate $x$ instead of the reduced coordinate $y$.
} 
This "seeding" function is then assigned to the first row of a basis set array chi $(1,:)$. The remainder of the basis set is constructed by replicating and circularly shifting the seeding function along the coordinate $\mathrm{x}$ with a userdefined step size step until the entire definition range has been spanned. In essence, the $i$-th basis function in the basis set array chi is produced by:

$$
\operatorname{chi}(i,:)=\operatorname{CIRCSHIFT}(\operatorname{ch} i(1,:),(i-1) * \text { step, } 2) \text {; }
$$

Through the use of the circshift command, the shifted vectors "wrap around" their edges, ensuring that the basis function amplitudes are evenly distributed along the full coordinate interval.

The resulting basis set is orthogonalized by means of diagonalizing its overlap matrix S:

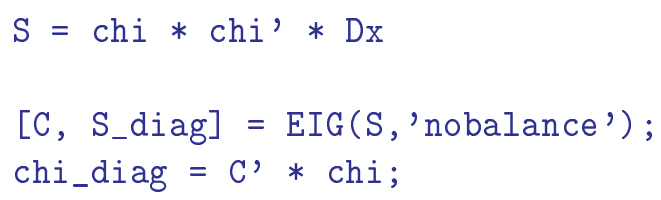

The functions belonging to eigenvalues below a certain threshold (default $10^{-5}$ ) are discarded. This improves numerical stability and eliminates negative overlap elements, which would produce complex-valued functions. The remaining orthogonal basis functions are normalized, and the overlap matrix is re-computed:

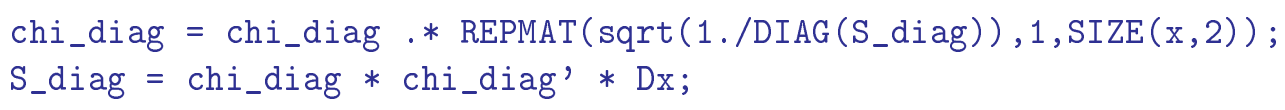

For diagnostics purposes, the largest residual off-diagonal element and the root-mean-square error of all off-diagonal elements are calculated.

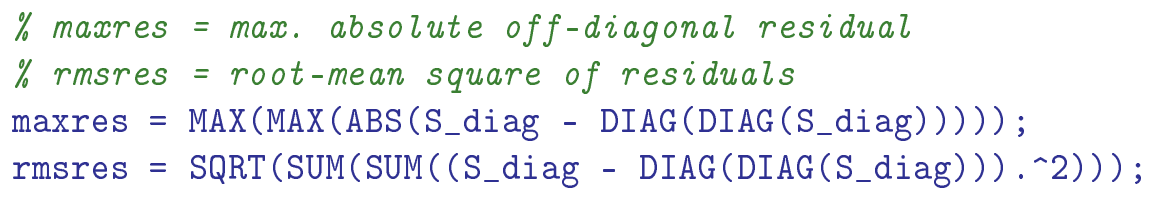

If maxres is larger than a hard-coded value $\left(10^{-10}\right)$, orthonormalization is re-attempted with the new basis set until it falls below this threshold. Typically, the two diagnostics measures are on the order of $10^{-14}$ to $10^{-15}$ after the first orthonormalization attempt if the basis set is well-constructed (i.e., the Gaussian functions are not too narrow for the numeric grid, and spaced not too far apart). The array chi is overwritten with the finalized basis set of dimension dim.

After preparing the basis set, the Hamiltonian matrix can be calculated, for which a numeric potential energy vector $\mathrm{V}$ and a reduced mass $\mathrm{mu}^{\dagger}$ of the

\footnotetext{
${ }^{\dagger}$ Not to be confused with the parameter for the basis set definition above.
} 
vibrational problem have to be supplied. For the kinetic energy operator, numerical second derivatives are calculated from finite differences in the basis functions through circular shifts of the basis function array chi along the $x$ coordinate, again wrapping around at the margins of the numeric vectors.

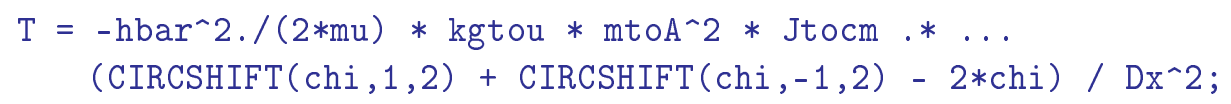

While differentiation could in principle also be carried out by a more straightforward DIFF (chi, $x, 2)$ call, the CIRCSHIFT approach is a more correct formalism for periodic problems such as the torsion/rotation motion in the methanol monomer, since DIFF naturally clips a vector to a smaller size. The results published in Reference 16 were obtained without this circularshift differentiation, but the impact of the code modification on the calculated eigenstates was found to be non-noticeable in these linear problems, at least if the coordinate interval is chosen large enough so that the amplitudes of all (relevant) wavefunctions vanish at its margins.

Construction of the potential energy operator is done in a straightforward way by expanding the numeric potential energy vector $v$ to match the dimension dim of the basis set.

$$
\mathrm{U}=\operatorname{REPMAT}(\mathrm{V}, \operatorname{dim}, 1) \cdot * \operatorname{ch} i \text {; }
$$

To finalize the elements of the Hamiltonian matrix, a temporary vector $\mathrm{H}_{-}$temp is calculated by co-adding the $\mathrm{T}$ and $\mathrm{U}$ matrices, forming the elementwise product from the left with the array chi, and numerically integrating along the coordinate $\mathrm{x}$. Through successively shifting the basis set array chi upwards in its vertical dimension, different basis functions are combined on either side of the corresponding bra-ket terms, yielding the off-diagonal Hamiltonian elements. The Hamiltonian matrix $\mathrm{H}$ is iteratively built up from expanding the temporary vectors $H_{-}$temp to diagonal matrix forms and adding them to the appropriate off-diagonals of $\mathrm{H}$.

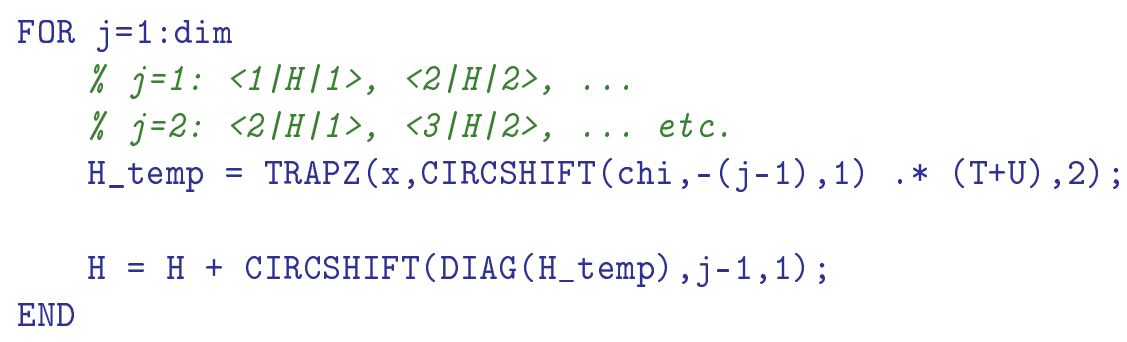

A slight non-symmetry in the finalized Hamiltonian matrix is generally observed, most likely due to remaining numerical inaccuracies. To diagnose the impact of this symmetry breaking, the eigenvalues are calculated twice: first with the original matrix $\mathrm{H}$, then after averaging all equivalent 
off-diagonal elements $H_{i j}$ and $H_{j i}$. The eigenvalues and eigenvectors of $\mathrm{H}$ are sorted in ascending order and stored.

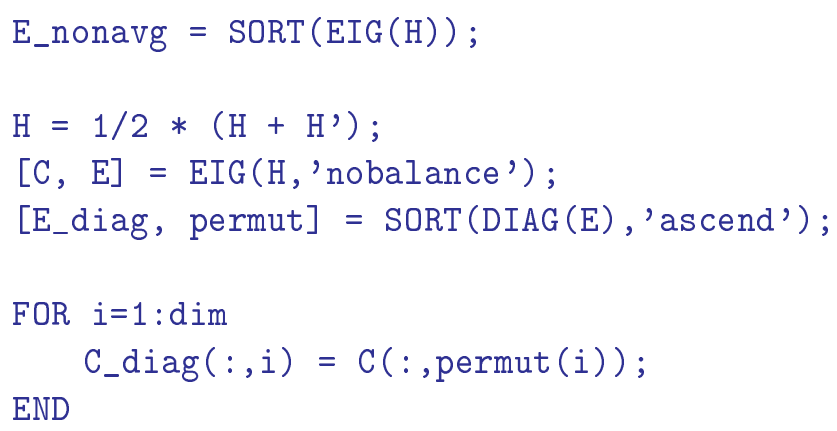

If any of the resulting eigenvalues (or the $n$ lowest ones, per optional user request) are found to differ by more than a pre-set parameter $\left(10^{-3} \mathrm{~cm}^{-1}\right)$ between the symmetrized and non-symmetrized result sets, a warning is issued; this can typically be rectified by either extending the coordinate space, increasing the numeric resolution, or adjusting the available parameters of the distributed Gaussian functions (i.e., their width and spacing).

Construction of the wavefunctions is straightforward, using the eigenvectors of the symmetrized Hamiltonian:

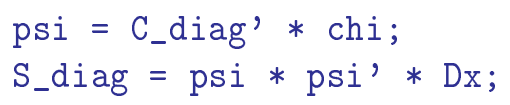

Again, the overlap matrix is diagnosed by means of maxres and rmsres as before during construction of the basis set, triggering a warning if any offdiagonal overlap element surmounts a threshold. The calculations are finished by printing the $\mathrm{S}$ diagnostics and energy levels to the screen.

\section{B.2 Parameters}

\section{B.2.1 Methanol Monomer Torsion}

In the torsional monomer calculations in Section 2.1, the coordinate $\mathrm{x}$ obtains the character of an angular displacement, and the reduced mass of the vibrational/rotational problem is replaced by an effective moment of inertia. The nomenclature of both quantities is retained here for consistency. In this formulation, mu can be determined from the parameters $F=\hbar /(4 \pi c \cdot \mathrm{mu}$ ) (see Section 2.1), which are given by Rueda et al. in Reference 108. The parameters for the potential functions as per Equation (2.3), and the values of $F$ and $\mathrm{mu}$ are given in Table B.1. Small offsets of the potential functions, $V_{\mathrm{s}}$, were deduced from minimizing the root-mean-square errors (RMSE) of the 
Table B.1: Hamiltonian and potential parameters for the methanol monomer torsion calculations (Eqs. (2.2) and (2.3)) used within this work, from Ref. 108. All data except for $\mathrm{mu}$ in $\mathrm{cm}^{-1}$.

\begin{tabular}{rrrrrrr}
\hline \multicolumn{1}{r}{$V_{3}$} & $V_{6}$ & $V_{\mathrm{s}}$ & $\mathrm{RMSE}$ & $F$ & $\mathrm{mu} / \mathrm{u}$ \\
\hline $\mathrm{v}_{\mathrm{S}}=0$ & 373.59 & -1.60 & -0.2 & 0.68 & 27.65 & 0.610 \\
1 & 410.20 & -1.09 & 3767.6 & 0.57 & 26.83 & 0.628 \\
2 & 446.41 & -2.85 & 7186.6 & 0.66 & 25.79 & 0.654 \\
\hline
\end{tabular}

lowest torsional levels up to $\mathrm{v}_{\mathrm{t}}=2$ with the experimental literature data. Naturally, the coordinate $\mathrm{x}$ was defined over the full circular range between $\pm \pi$ :

$$
\mathrm{x}=[-\mathrm{pi}: \mathrm{pi} / 5000:+\mathrm{pi}]
$$

The basis set was constructed as detailed above with the parameters $\mathrm{mu}=$ $1 \mathrm{u}$, omega $=400 \mathrm{~cm}^{-1}$, and a step size of 5 .

The chosen range and gridding in $\mathrm{x}$ determines all numeric vectors along this coordinate to be of length 10001. For the localized problem, the two potential wells at $\pm \pi / 3$ were replaced with the maximum value of the potential function to build the plateau potentials:

$$
\begin{aligned}
& \mathrm{V}(1: 3334)=\operatorname{MAX}(\mathrm{V}) ; \\
& \mathrm{V}(6668: 10001)=\operatorname{MAX}(\mathrm{V}) ;
\end{aligned}
$$

The energy levels in these potentials were then calculated with the same parameters and basis sets as before.

\section{B.2.2 OH Stretching Vibrations}

For the $\mathrm{OH}$ stretching vibration problems in the methanol monomer, the methanol dimer donor, and methanol-ethene, the normal modes and reduced masses were obtained from a standard vibrational analysis for each structure at the LCCSD(T0*)-F12a(int)/VDZ-F12 level of theory. Potential energy curves along these vibrational coordinates were built from a number of single-point energies at displacements up to $Q=-0.3$ and $+0.9 \AA$, fitted with a modified Morse potential as per Equation (2.7),

$$
V(Q)=C\left[1-\exp \left(-\sum_{i=1}^{5} b_{i} Q^{i}\right)\right]^{2} .
$$


Table B.2: Hamiltonian and potential parameters for the 1-D OH stretching calculations of the methanol monomer ("M"), and the methanol dimer ("MM") and methanol-ethene ("ME") donors (Eq. (2.7)). The parameters $b_{i}$ are given in units of $\AA^{-i}$.

\begin{tabular}{lrrrrrrr}
\hline & $C / \mathrm{cm}^{-1}$ & $b_{1}$ & $b_{2}$ & $b_{3}$ & $b_{4}$ & $b_{5}$ & $\mathrm{mu} / \mathrm{u}$ \\
\hline $\mathrm{M}$ & 46907.9 & 2.2426 & -0.2873 & 0.3649 & -0.2575 & -0.1303 & 1.0667 \\
$\mathrm{MM}$ & 1635843.2 & 0.3676 & -0.4500 & 0.3209 & -0.2468 & 0.1087 & 1.0674 \\
$\mathrm{ME}$ & 78825.4 & 1.7106 & -0.6880 & 0.1737 & -0.2458 & 0.1830 & 1.0664 \\
\hline
\end{tabular}

Some multistabilities were found in these fits, but since there is no direct physical meaning attached to the fitted parameters, they were still judged to be satisfactory on grounds of the overall 1 to $2 \mathrm{~cm}^{-1}$ residual root-meansquare errors (RMSE). The results for $C$ and $b_{1}$ through $b_{5}$ are listed in Table B.2, together with the values of mu from normal-mode analyses. For all systems, the coordinate $\mathrm{x}$ was defined in $0.0001 \AA$ steps between -0.5 and $+0.9 \AA$ :

$$
\mathrm{x}=[-0.5: 0.0001: 0.9] ;
$$

The basis set was constructed with parameters omega $=10000 \mathrm{~cm}^{-1}$ and step $=25$, using the same mu values as for the respective vibrational problems themselves. 


\section{Appendix C}

\section{MOLPRO Inputs and LMOMO Calculations}

For all MOLPRO calculations, increased convergence criteria were used for the integral accuracy in the SCF routine and global energy calculations, using the ACCU, 16 directive for the Hartree-Fock program, and a global GTHRESH, ENERGY=1E-10 definition. The OPT=TIGHT geometry convergence criteria of the GAUSSIAN 09 package were mimicked in MOLPRO by adding

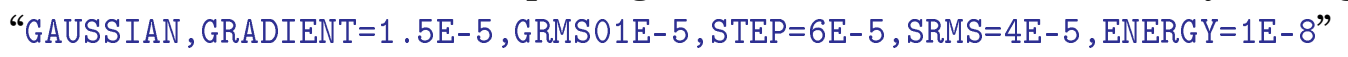
to the OPTG routine call.

If local electron correlation is employed in Coupled Cluster approaches, the customary perturbative triples treatment in $\operatorname{CCSD}(\mathrm{T})$ must in principle be carried out iteratively to be exact. However, the errors that arise from simply calculating the triples in a single iteration are typically negligible, which is designated "(T0)" and was used per default for all LCCSD(T) calculations presented throughout this work. ${ }^{71}$ To aid the robustness of the automated domain constructions, the two most diffuse basis functions were deleted from each atomic center by using the PIPEK, DELETE=2 command. Typical threshold values for the NPA domain-selection criterion (governed by the NPASEL parameter) were then between 0.03 and 0.05 . For calculations on anisole, the MERGEDOM=1 command was used to merge the non-unique localized domains in the aromatic $\pi$ system.

\section{C.1 Explicit Correlation}

Due to a programming bug in version 2012.1, explicitly correlated LCCSD(T)-F12 calculations do not honor the request for scaling the perturbative triples (SCALE_TRIP=1 keyword). Thus, a series of energy defini- 
tions was added manually in all calculations to step-wise build the desired LCCSD(T0*)-F12a energy:

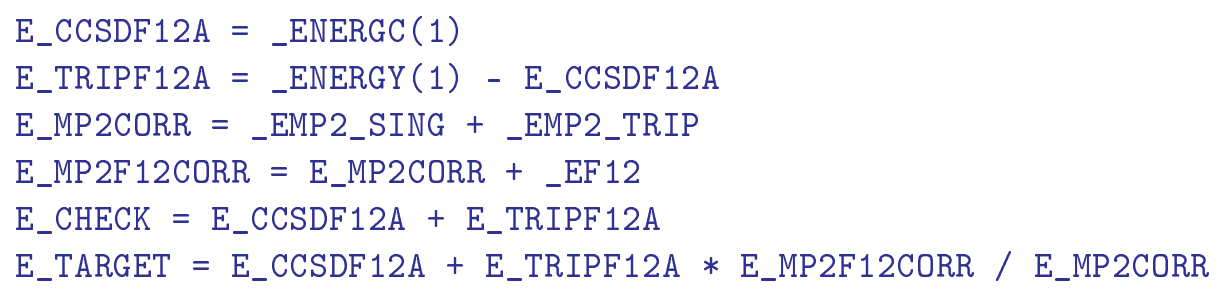

These definitions specify:

- E_CCSDF12A: full LCCSD-F12a "base" energy, including the CABScorrected SCF reference as described in Section 1.3.1;

- E_TRIPF12A: unscaled triples contributions, extracted from the LCCSD(T)-F12a energy by subtracting E_CCSDF12A;

- E_MP2CORR: LMP2 correlation energy as calculated from the LMP2 singles and triples contributions;

- E_MP2F12CORR: LMP2-F12 correlation energy, obtained from adding the effect of explicit correlation to the correlation energy E_MP2CORR;

- E_CHECK: full LCCSD(T0)-F12a energy (including SCF reference), used as a consistency check against the output provided by the program;

- E_TARGET: final LCCSD $\left(\mathrm{T}^{*}\right)-\mathrm{F} 12 \mathrm{a}$ energy, using the ratio of E_MP2F12CORR and E_MP2CORR to scale the E_TRIPF12A triples contributions.

\section{C.2 Spin-Component Scaling}

Using Grimme's Spin-Component Scaling (SCS) ${ }^{125}$ in MP2 calculations is possible in MOLPRO by means of the SCSGRD=1 keyword. Unfortunately, the program code appears to recognize this command only in the local, but not the canonical formalism. A workaround for enabling canonical SCS-MP2 calculations is provided by calling the local LMP2 program and extending the domains to encompass the entire molecule, thereby fully undoing the localization approach:

$\{\mathrm{DF}-\mathrm{LMP} 2, \mathrm{BASIS}=\mathrm{avtz}, \mathrm{SCSGRD}=1$

LOCAL , THRBP $=1.0\}$ 


\section{C.3 LMOMO Calculations}

LMOMO calculation in MOLPRO are requested by including a "REGION" keyword in the input block of the local calculation command. An exemplary input for the methanol dimer would take the form:

REGION , CCSD (T) , DEFAULT=MP 2, H6 , TYPE=INCLUSIVE

REGION , CCSD (T) , DEFAULT=MP2, 08, TYPE=EXCLUSIVE

The INCLUSIVE and EXCLUSIVE cards control whether all domains containing the given atomic centers are to be treated at specified given level of theory, or only those domains which are exclusively located on these centers. In the above example, the first line elevates the electron pair comprising the donor $\mathrm{OH}$ bond to the $\mathrm{CCSD}(\mathrm{T})$ level, since the corresponding domain contains the specified $\mathrm{H} 6$ atom. The effect of the second line is to also include the two lone pairs of the acceptor 08 atom in the $\operatorname{CCSD}(\mathrm{T})$ region, but not the adjacent $\mathrm{CO}$ and $\mathrm{OH}$ bonds (since they contain more than just this atomic center).

Details about the LMOMO calculations on the methanol dimer (Section 2.3) are given below in tabulated form. The relevant atomic centers are labeled 02 and $\mathrm{H} 6$ for the donor $\mathrm{OH}$ group, and 08 and $\mathrm{H} 12$ for the acceptor $\mathrm{OH}$ group. The methanol-ethene LCCSD(T0)(int):LMP2 calculation included in Table 3.1 was carried out with an INCLUSIVE treatment of the donor $\mathrm{O}$ and both acceptor $\mathrm{C}$ atoms. This scheme again correlates the $\mathrm{C}-\mathrm{O} / \mathrm{O}-\mathrm{H}$ bonds and $\mathrm{O}$ lone pairs in the methanol donor, as well as the entire ethene acceptor and all intermolecular pairs, at the $\operatorname{CCSD}(\mathrm{T})$ level. 
Table C.1: LMOMO calculations of the methanol dimer presented in Fig. 2.8 (top) and Tab. 2.5 (bottom). The treatment of electron pairs is indicated either at the CCSD(T0) ("CC") or MP2 level, with "(int)" referring to all intermolecular pairs.

\begin{tabular}{|c|c|c|c|c|c|c|}
\hline & & \multicolumn{5}{|c|}{ Figure 2.8} \\
\hline & & "a" & $" b "$ & "c" & “d” & “e” \\
\hline INCLUSIVE & & - & H6 & $\mathrm{H} 6,08$ & 02,08 & 02,08 \\
\hline EXCLUSIVE & & - & - & - & - & - \\
\hline \multirow[t]{3}{*}{ Donor: } & $\mathrm{O}-\mathrm{H}$ bond & MP2 & $\mathrm{CC}$ & $\mathrm{CC}$ & $\mathrm{CC}$ & $\mathrm{CC}$ \\
\hline & O lone pairs & MP2 & MP2 & MP2 & $\mathrm{CC}$ & $\mathrm{CC}$ \\
\hline & $\mathrm{C}-\mathrm{O}$ bond & MP2 & MP2 & MP2 & MP2 & MP2 \\
\hline \multirow[t]{3}{*}{ Acceptor: } & $\mathrm{O}-\mathrm{H}$ bond & MP2 & MP2 & $\mathrm{CC}$ & $\mathrm{CC}$ & $\mathrm{CC}$ \\
\hline & O lone pairs & MP2 & MP2 & $\mathrm{CC}$ & $\mathrm{CC}$ & $\mathrm{CC}$ \\
\hline & C-O bond & MP2 & MP2 & MP2 & MP2 & MP2 \\
\hline \multirow[t]{3}{*}{ (int) } & & MP2 & MP2 & MP2 & MP2 & $\mathrm{CC}$ \\
\hline & & \multicolumn{5}{|c|}{ Table 2.5} \\
\hline & & \multicolumn{2}{|c|}{ "LCCSD(T0)" } & \multicolumn{2}{|c|}{ “LMOMO” } & "LMOMO + C-O" \\
\hline \multicolumn{2}{|l|}{ INCLUSIVE } & \multicolumn{2}{|l|}{-} & \multicolumn{2}{|c|}{$\mathrm{H} 6, \mathrm{H} 12$} & 02,08 \\
\hline \multicolumn{2}{|l|}{ EXCLUSIVE } & \multicolumn{2}{|l|}{-} & \multicolumn{2}{|c|}{02,08} & - \\
\hline \multirow[t]{3}{*}{ Donor: } & $\mathrm{O}-\mathrm{H}$ bond & \multicolumn{2}{|l|}{$\mathrm{CC}$} & \multicolumn{2}{|l|}{$\mathrm{CC}$} & $\mathrm{CC}$ \\
\hline & O lone pairs & \multicolumn{2}{|l|}{$\mathrm{CC}$} & \multicolumn{2}{|l|}{$\mathrm{CC}$} & $\mathrm{CC}$ \\
\hline & C-O bond & \multicolumn{2}{|l|}{$\mathrm{CC}$} & \multicolumn{2}{|c|}{ MP2 } & $\mathrm{CC}$ \\
\hline \multirow[t]{3}{*}{ Acceptor: } & $\mathrm{O}-\mathrm{H}$ bond & $\mathrm{CC}$ & & \multicolumn{2}{|l|}{$\mathrm{CC}$} & $\mathrm{CC}$ \\
\hline & O lone pairs & $\mathrm{CC}$ & & \multicolumn{2}{|l|}{$\mathrm{CC}$} & $\mathrm{CC}$ \\
\hline & C-O bond & $\mathrm{CC}$ & & \multicolumn{2}{|c|}{ MP2 } & $\mathrm{CC}$ \\
\hline (int) & \multicolumn{6}{|c|}{ as specified } \\
\hline
\end{tabular}




\section{Appendix D}

\section{VPT2 Stability Tests}

As mentioned in Section 1.3.3, several numerical instabilities were discovered in the VPT2 calculations in GAUSSIAN 09. The first one exclusively relates to DFT calculations, which are sensitive to the underlying input structures; the second one also impacts $a b$ initio methods and occurs when the input structures are re-read from a checkpoint file instead of directly taken from a preceding geometry optimization. To demonstrate and assess these instabilities, a number of nominally equivalent calculations was conducted in Reference 17 for the methanol monomer, methanol homodimer, and methanol-ethene. The results are repeated below, including an additional MP2 calculation "II" for methanol-ethene.

In the following tables, the anharmonic calculations are divided into four categories, which should in principle all yield the same results: (I) An original geometry optimization and anharmonic calculation; (II) using the optimized structure from the checkpoint file of the first calculation (GEOM=CHECKPOINT), re-optimized using the OPT=TIGHT setting; (III) starting at a different input structure, using OPT=TIGHT; and (IV) using the structures stored in the checkpoint file of calculation (I) without re-optimization. All DFT calculations were done with the INT=ULTRAFINE grid setting. The data reveal distinct variations in the DFT results for all structures, which are larger at the B3LYP-D3 that at the B2PLYP-D3 level. In addition to the stretching and libration modes, the dimer data also includes selected data on the second-lowest harmonic vibration (subscripts "2") that represents the hindered torsion of the acceptor methanol molecule, since it provides a striking measure for the robustness-or lack thereof-in these calculations. In methanol-ethene, the torsion of the ethene acceptor around the $\mathrm{OH} \cdots \pi$ hydrogen bond is included for the same purpose. 
Table D.1: VPT2 stability tests for the methanol monomer.

\begin{tabular}{r|rrr|rrrr|rrrrr}
\hline & MP2 & & & \multicolumn{3}{|c|}{ B2PLYP-D3 } & & & \multicolumn{2}{|c}{ B3LP-D3 } & & \\
& I & II & IV & I & II & III & IV & I & II & III & IV \\
\hline$\omega_{\mathrm{s}}$ & 3882 & 3882 & 3882 & 3858 & 3858 & 3858 & 3858 & 3829 & 3829 & 3829 & 3829 \\
$\tilde{v}_{\mathrm{s}}$ & 3706 & 3706 & 3706 & 3674 & 3674 & 3675 & 3674 & 3645 & 3645 & 3647 & 3645 \\
$\omega_{\mathrm{s}}$ & 309 & 309 & 309 & 307 & 307 & 307 & 307 & 306 & 306 & 306 & 306 \\
$\tilde{v}_{\mathrm{t}}$ & 257 & 256 & 256 & 241 & 243 & 243 & 242 & 233 & 236 & 234 & 232 \\
& & & & & & & & & & & \\
$\sum x_{\mathrm{s}, i}$ & -21 & -21 & -21 & -24 & -24 & -22 & -24 & -22 & -22 & -17 & -22 \\
$\sum^{\prime} x_{\mathrm{s}, j}$ & -30 & -30 & -30 & -28 & -28 & -28 & -28 & -26 & -25 & -26 & -26 \\
& & & & & & & & & & & \\
$x_{\mathrm{s}, \mathrm{s}}$ & -83 & -83 & -83 & -86 & -86 & -86 & -86 & -87 & -87 & -87 & -87 \\
$x_{\mathrm{s}, \mathrm{t}}$ & +9 & +9 & +9 & +4 & +4 & +5 & +4 & +3 & +4 & +8 & +3 \\
$x_{\mathrm{t}, \mathrm{t}}$ & -30 & -31 & -31 & -35 & -34 & -34 & -35 & -37 & -36 & -37 & -37 \\
$\sum^{\prime} x_{\mathrm{t}, j}$ & +9 & +8 & +9 & +3 & +5 & +5 & +4 & -2 & 0 & -3 & -2 \\
\hline
\end{tabular}


Table D.2: VPT2 stability tests for the methanol dimer. Subscripts "2" denote the harmonically second-lowest vibration which serves as a particularly sensitive accuracy indicator. The last line further includes the summed couplings of the librational mode to the seven lowest vibrations.

\begin{tabular}{r|rrr|rrrr|rrrr}
\hline & MP2 & & & \multicolumn{3}{|c|}{ B2PLYP-D3 } & & & B3LYP-D3 & & \\
& I & II & IV & I & II & III & IV & I & II & III & IV \\
\hline$\omega_{\mathrm{s}}$ & 3740 & 3740 & 3740 & 3722 & 3722 & 3722 & 3722 & 3687 & 3687 & 3687 & 3687 \\
$\tilde{v}_{\mathrm{s}}$ & 3571 & 3571 & 3571 & 3554 & 3557 & 3559 & 3557 & 3520 & 3523 & 3507 & 3514 \\
$\omega_{1}$ & 699 & 699 & 699 & 697 & 697 & 697 & 697 & 700 & 700 & 700 & 700 \\
$\tilde{v}_{1}$ & 592 & 592 & 594 & 589 & 594 & 613 & 600 & 586 & 596 & 556 & 575 \\
$\omega_{2}$ & 56 & 56 & 56 & 60 & 60 & 60 & 60 & 53 & 53 & 53 & 53 \\
$\tilde{v}_{2}$ & 36 & 36 & 16 & 11 & 108 & 6 & 23 & 2 & $10 \mathrm{i}$ & $12 \mathrm{i}$ & $10 \mathrm{i}$ \\
$(2 \tilde{v})_{2}$ & 43 & 43 & $2 \mathrm{i}$ & $8 \mathrm{i}$ & 207 & $20 \mathrm{i}$ & 17 & $30 \mathrm{i}$ & $60 \mathrm{i}$ & $55 \mathrm{i}$ & $53 \mathrm{i}$ \\
$\tilde{v}_{\mathrm{s}, 1}$ & 4222 & 4222 & 4225 & 4200 & 4209 & 4230 & 4215 & 4164 & 4178 & 4120 & 4147 \\
& & & & & & & & & & & \\
$\sum x_{\mathrm{s}, i}$ & +68 & +68 & +68 & +73 & +79 & +83 & +79 & +83 & +90 & +58 & +72 \\
$\sum^{\prime} x_{\mathrm{s}, j}$ & +9 & +9 & +9 & +15 & +22 & +26 & +22 & +25 & +32 & +0 & +14 \\
& & & & & & & & & & & \\
$x_{\mathrm{s}, \mathrm{s}}$ & -102 & -102 & -102 & -102 & -102 & -102 & -102 & -104 & -104 & -104 & -104 \\
$x_{\mathrm{s}, 1}$ & +59 & +59 & +59 & +58 & +58 & +58 & +58 & +58 & +58 & +58 & +58 \\
$x_{1,1}$ & -46 & -46 & -46 & -43 & -43 & -43 & -43 & -45 & -45 & -46 & -46 \\
$\sum x_{1,1 \ldots 7}$ & -71 & -71 & -66 & -80 & -71 & -40 & -61 & -85 & -68 & -134 & -103 \\
\hline
\end{tabular}


Table D.3: VPT2 stability tests for the mixed methanol-ethene dimer. Subscripts " 1 " denote the torsional vibration of the ethene molecule around the $\mathrm{OH} \cdots \pi$ hydrogen bond.

\begin{tabular}{r|rrr|rr|rr}
\hline & MP2 & & & \multicolumn{3}{|c|}{ B2PLYP-D3 } & \multicolumn{2}{|c}{ B3LYP-D3 } \\
& I & II & IV & I & III & I & III \\
\hline$\omega_{\mathrm{s}}$ & 3823 & 3823 & 3823 & 3805 & 3805 & 3776 & 3776 \\
$\tilde{v}_{\mathrm{s}}$ & 3652 & 3652 & 3652 & 3633 & 3622 & 3601 & 3604 \\
$\omega_{1}$ & 448 & 448 & 448 & 431 & 431 & 425 & 425 \\
$\tilde{v}_{1}$ & 372 & 375 & 372 & 383 & 295 & 324 & 346 \\
$\omega_{1}$ & 15 & 15 & 15 & 19 & 19 & 12 & 12 \\
$\tilde{v}_{1}$ & 22 & 18 & 22 & 14 & $45 \mathrm{i}$ & $206 \mathrm{i}$ & $16 \mathrm{i}$ \\
& & & & & & & \\
$\sum x_{\mathrm{s}, i}$ & +12 & +12 & +12 & +20 & -2 & +19 & +23 \\
$\sum^{\prime} x_{\mathrm{s}, j}$ & -4 & -4 & -4 & +6 & -15 & +1 & +5 \\
& & & & & & & \\
$x_{\mathrm{s}, \mathrm{s}}$ & -88 & -88 & -88 & -91 & -91 & -92 & -92 \\
$x_{\mathrm{s}, 1}$ & +17 & +17 & +17 & +14 & +13 & +18 & +18 \\
\hline
\end{tabular}




\section{Bibliography}

[1] T. Häber, U. Schmitt, and M. A. Suhm. FTIR-spectroscopy of molecular clusters in pulsed supersonic slit-jet expansions. Phys. Chem. Chem. Phys., 1:5573-5582, 1999.

[2] R. E. Smalley, L. Wharton, and D. H. Levy. Molecular optical spectroscopy with supersonic beams and jets. Acc. Chem. Res., 10:139-145, 1977.

[3] M. Herman, R. Georges, M. Hepp, and D. Hurtmans. High resolution Fourier transform spectroscopy of jet-cooled molecules. Int. Rev. Phys. Chem., 19:277-325, 2000.

[4] E. Arunan, G. R. Desiraju, R. A. Klein, J. Sadlej, S. Scheiner, I. Alkorta, D. C. Clary, R. H. Crabtree, J. J. Dannenberg, P. Hobza, H. G. Kjaergaard, A. C. Legon, B. Mennucci, and D. J. Nesbitt. Defining the hydrogen bond: An account (IUPAC Technical Report). Pure Appl. Chem., 83:1619-1636, 2011.

[5] E. Arunan, G. R. Desiraju, R. A. Klein, J. Sadlej, S. Scheiner, I. Alkorta, D. C. Clary, R. H. Crabtree, J. J. Dannenberg, P. Hobza, H. G. Kjaergaard, A. C. Legon, B. Mennucci, and D. J. Nesbitt. Definition of the hydrogen bond (IUPAC Recommendations 2011). Pure Appl. Chem., 83:1637-1641, 2011.

[6] R. M. Badger and S. H. Bauer. Spectroscopic Studies of the Hydrogen Bond. II. The Shift of the O-H Vibrational Frequency in the Formation of the Hydrogen Bond. J. Chem. Phys., 5:839-851, 1937.

[7] A. V. Iogansen. Direct proportionality of the hydrogen bonding energy and the intensification of the stretching $v(\mathrm{XH})$ vibration in infrared spectra. Spectrochim. Acta A, 55:1585-1612, 1999. 
[8] C. Emmeluth, V. Dyczmons, and M. A. Suhm. Tuning the hydrogen bond donor/acceptor isomerism in jet-cooled mixed dimers of aliphatic alcohols. J. Phys. Chem. A, 110:2906-2915, 2006.

[9] E. T. Nibbering, J. Dreyer, O. Kühn, J. Bredenbeck, P. Hamm, and T. Elsaesser. Vibrational dynamics of hydrogen bonds. In O. Kühn and L. Wöste (editors), Analysis and control of ultrafast photoinduced reactions, volume 87 of Springer series in chemical physics, pages 619687. Springer, Berlin and New York, 2007.

[10] J.-J. Péron. The anharmonicity of the $\mathrm{OH}$ stretching vibration of hydrogen bonded methanol in binary systems. J. Chem. Phys., 65:31533157, 1976.

[11] F. J. Lovas and H. Hartwig. The Microwave Spectrum of the Methanol Dimer for $K=0$ and 1 States. J. Mol. Spectrosc., 185:98-109, 1997.

[12] J. P. Perchard and Z. Mielke. Anharmonicity and hydrogen bonding. I. A near-infrared study of methanol trapped in nitrogen and argon matrices. Chem. Phys., 264:221-234, 2001.

[13] R. Wugt Larsen, P. Zielke, and M. A. Suhm. Hydrogen-bonded OH stretching modes of methanol clusters: A combined IR and Raman isotopomer study. J. Chem. Phys., 126:194307, 2007.

[14] F. Kollipost, K. Papendorf, Y.-F. Lee, Y.-P. Lee, and M. A. Suhm. Alcohol dimers - how much diagonal $\mathrm{OH}$ anharmonicity? Phys. Chem. Chem. Phys., 16:15948-15956, 2014.

[15] M. Heger, M. A. Suhm, and R. A. Mata. Communication: Towards the binding energy and vibrational red shift of the simplest organic hydrogen bond: harmonic constraints for methanol dimer. J. Chem. Phys., 141:101105, 2014.

[16] M. Heger, R. A. Mata, and M. A. Suhm. Soft hydrogen bonds to alkenes: The methanol-ethene prototype under experimental and theoretical scrutiny. Chem. Sci., 6:3738-3745, 2015.

[17] M. Heger, J. Andersen, M. A. Suhm, and R. Wugt Larsen. The donor $\mathrm{OH}$ stretching-libration dynamics of hydrogen-bonded methanol dimers in cryogenic matrices. Phys. Chem. Chem. Phys., 18:37393745, 2016. 
[18] L. G. Bonner. The Vibrational Spectrum of Water Vapor. Phys. Rev., 46:458-464, 1934.

[19] G. Herzberg. Molecular spectra and molecular structure. R.E. Krieger Pub. Co, Malabar, Fla., 2nd edition, 1989-1991.

[20] J. L. Dunham. Intensities of Vibration-Rotation Bands with Special Reference to Those of HCl. Phys. Rev., 35:1347-1354, 1930.

[21] J. M. Hollas. Modern spectroscopy. Wiley, Chichester, 4th edition, 2004.

[22] B. L. Crawford and H. L. Dinsmore. Vibrational Intensities. I. Theory of Diatomic Infra-Red Bands. J. Chem. Phys., 18:983-987, 1950.

[23] B. L. Crawford and H. L. Dinsmore. Errata: Vibrational Intensities. I. Theory of Diatomic Infra-Red Bands. J. Chem. Phys., 18:1682-1683, 1950.

[24] E. B. Wilson, J. C. Decius, and P. C. Cross. Molecular vibrations: The theory of infrared and Raman vibrational spectra. Dover Publications, New York, 1980.

[25] C. Sándorfy. Hydrogen bonding: How much anharmonicity? J. Mol. Struct., 790:50-54, 2006.

[26] T. Di Paolo, C. Bourdéron, and C. Sandorfy. Model Calculations on the Influence of Mechanical and Electrical Anharmonicity on Infrared Intensities: Relation to Hydrogen Bonding. Can. J. Chem., 50:31613166, 1972.

[27] R. C. Herman and K. E. Shuler. Vibrational Intensities in Diatomic Infrared Transitions. The Vibrational Matrix Elements for CO. J. Chem. Phys., 22:481-490, 1954.

[28] H. G. Kjaergaard, G. R. Low, T. W. Robinson, and D. L. Howard. Calculated $\mathrm{OH}-S t r e t c h i n g$ Vibrational Transitions in the Water-Nitrogen and Water-Oxygen Complexes. J. Phys. Chem. A, 106:8955-8962, 2002.

[29] T. Scharge, D. Luckhaus, and M. A. Suhm. Observation and quantification of the hydrogen bond effect on $\mathrm{O}-\mathrm{H}$ overtone intensities in an alcohol dimer. Chem. Phys., 346:167-175, 2008. 
[30] A. Kantrowitz and J. Grey. A High Intensity Source for the Molecular Beam. Part I. Theoretical. Rev. Sci. Instrum., 22:328-332, 1951.

[31] M. D. Morse. Supersonic Beam Sources. In F. B. Dunning and R. G. Hulet (editors), Atomic, Molecular, and Optical Physics: Atoms and Molecules, volume 29, Part B of Experimental Methods in the Physical Sciences, pages 21-47. Academic Press, San Diego, Calif., 1996.

[32] J. Arnó and J. W. Bevan. Infrared spectroscopy in supersonic free jets and molecular beams. In J. M. Hollas and D. Phillips (editors), Jet spectroscopy and molecular dynamics, pages 29-73. Springer, Dordrecht, 1995.

[33] O. F. Hagena. Cluster Formation in Expanding Supersonic Jets: Effect of Pressure, Temperature, Nozzle Size, and Test Gas. J. Chem. Phys., 56:1793-1802, 1972.

[34] O. F. Hagena. Nucleation and growth of clusters in expanding nozzle flows. Surf. Sci., 106:101-116, 1981.

[35] W. Schöllkopf and J. P. Toennies. Nondestructive Mass Selection of Small van der Waals Clusters. Science, 266:1345-1348, 1994.

[36] R. E. Smalley. Laser spectroscopy of supersonic molecular beams: Application to the $\mathrm{NO}_{2}$ spectrum. J. Chem. Phys., 61:4363-4364, 1974.

[37] T. N. Wassermann and M. A. Suhm. Ethanol monomers and dimers revisited: a Raman study of conformational preferences and argon nanocoating effects. J. Phys. Chem. A, 114:8223-8233, 2010.

[38] M. Heger, K. E. Otto, R. A. Mata, and M. A. Suhm. Bracketing subtle conformational energy differences between self-solvated and stretched trifluoropropanol. Phys. Chem. Chem. Phys., 17:9899-9909, 2015.

[39] N. O. B. Lüttschwager and M. A. Suhm. Stretching and folding of 2-nanometer hydrocarbon rods. Soft Matter, 10:4885-4901, 2014.

[40] D. J. Nesbitt. High-Resolution, Direct Infrared Laser Absorption Spectroscopy in Slit Supersonic Jets: Intermolecular Forces and Unimolecular Vibrational Dynamics in Clusters. Annu. Rev. Phys. Chem., 45:367-399, 1994.

[41] K. Schwing and M. Gerhards. Combined Infrared/Ultraviolet Spectroscopy in Molecular Beam Experiments. Bunsen-Magazin, 16:116131, 2014. 
[42] I. León, R. Montero, A. Longarte, and J. A. Fernández. IR mass-resolved spectroscopy of complexes without chromophore: cyclohexanol.(H2O), $\mathrm{n}=1-3$ and cyclohexanol dimer. J. Chem. Phys., 139:174312, 2013.

[43] M. A. Suhm and F. Kollipost. Femtisecond single-mole infrared spectroscopy of molecular clusters. Phys. Chem. Chem. Phys., 15:1070210721, 2013.

[44] T. Häber, U. Schmitt, C. Emmeluth, and M. A. Suhm. Ragout-jet FTIR spectroscopy of cluster isomerism and cluster dynamics: from carboxylic acid dimers to $\mathrm{N}_{2} \mathrm{O}$ nanoparticles. Faraday Disc., 118:331359, 2001.

[45] J. B. Anderson. Separation of gas mixtures in free jets. AIChE J., 13:1188-1192, 1967.

[46] J. B. Anderson and P. Davidovits. Isotope separation in a "seeded beam". Science, 187:642-644, 1975.

[47] A. Miffre, M. Jacquey, M. Büchner, G. Trénec, and J. Vigué. Parallel temperatures in supersonic beams: ultracooling of light atoms seeded in a heavier carrier gas. J. Chem. Phys., 122:94308, 2005.

[48] N. Borho. Chirale Erkennung in Molekülclustern: Maßgeschneiderte Aggregation von $\alpha$-Hydroxyestern. Ph.D. thesis. Cuvillier, Göttingen, 2004.

[49] R. Wugt Larsen and M. A. Suhm. The benefits of alternation and alkylation: large amplitude hydrogen bond librational modes of alcohol trimers and tetramers. Phys. Chem. Chem. Phys., 12:8152-8157, 2010.

[50] F. Kollipost, R. Wugt Larsen, A. V. Domanskaya, M. Nörenberg, and M. A. Suhm. Communication: The highest frequency hydrogen bond vibration and an experimental value for the dissociation energy of formic acid dimer. J. Chem. Phys., 136:151101, 2012.

[51] S. Hesse. Schwache Wechselwirkungen zwischen organischen Molekülen: Strukturelle Vielfalt und ihre schwingungsspektroskopischen Auswirkungen. Ph.D. thesis. Cuvillier, Göttingen, 2010.

[52] M. Heger, J. Altnöder, A. Poblotzki, and M. A. Suhm. To $\pi$ or not to $\pi$ - how does methanol dock onto anisole? Phys. Chem. Chem. Phys., 17:13045-13052, 2015. 
[53] J. Ceponkus, P. Uvdal, and B. Nelander. Intermolecular vibrations of different isotopologs of the water dimer: Experiments and density functional theory calculations. J. Chem. Phys., 129:194306, 2008.

[54] J. Andersen, J. Heimdal, and R. Wugt Larsen. The influence of largeamplitude librational motion on the hydrogen bond energy for alcoholwater complexes. Phys. Chem. Chem. Phys., 17:23761-23769, 2015.

[55] O. Vahtras, J. Almlöf, and M. W. Feyereisen. Integral approximations for LCAO-SCF calculations. Chem. Phys. Lett., 213:514-518, 1993.

[56] F. Weigend. A fully direct RI-HF algorithm: Implementation, optimised auxiliary basis sets, demonstration of accuracy and efficiency. Phys. Chem. Chem. Phys., 4:4285-4291, 2002.

[57] A. P. Rendell and T. J. Lee. Coupled-cluster theory employing approximate integrals: An approach to avoid the input/output and storage bottlenecks. J. Chem. Phys., 101:400-408, 1994.

[58] H.-J. Werner, F. R. Manby, and P. J. Knowles. Fast linear scaling second-order Møller-Plesset perturbation theory (MP2) using local and density fitting approximations. J. Chem. Phys., 118:8149-8160, 2003.

[59] W. Klopper, F. R. Manby, S. Ten-No, and E. F. Valeev. R12 methods in explicitly correlated molecular electronic structure theory. Int. Rev. Phys. Chem., 25:427-468, 2006.

[60] T. Korona, D. Kats, M. Schütz, T. B. Adler, Y. Liu, and H.-J. Werner. Local Approximations for an Efficient and Accurate Treatment of Electron Correlation and Electron Excitations in Molecules. In R. Zaleśny, M. G. Papadopoulos, P. G. Mezey, and J. Leszczynski (editors), Linearscaling techniques in computational chemistry and physics: Methods and applications, volume 13 of Challenges and advances in computational chemistry and physics, pages 345-407. Springer, Dordrecht and London, 2011.

[61] P. Pulay. Localizability of dynamic electron correlation. Chem. Phys. Lett., 100:151-154, 1983.

[62] S. Saebø and P. Pulay. Local Treatment of Electron Correlation. Annu. Rev. Phys. Chem., 44:213-236, 1993. 
[63] S. Saebø, W. Tong, and P. Pulay. Efficient elimination of basis set superposition errors by the local correlation method: Accurate ab initio studies of the water dimer. J. Chem. Phys., 98:2170-2175, 1993.

[64] S. F. Boys and F. Bernardi. The calculation of small molecular interactions by the differences of separate total energies. Some procedures with reduced errors. Mol. Phys., 19:553-566, 1970.

[65] J. Kaminsky, R. A. Mata, H.-J. Werner, and F. Jensen. The accuracy of local MP2 methods for conformational energies. Mol. Phys., 106:1899_ 1906, 2008.

[66] G. Rauhut, A. El Azhary, F. Eckert, U. Schumann, and H.-J. Werner. Impact of local approximations on MP2 vibrational frequencies. Spectrochim. Acta A, 55:647-658, 1999.

[67] T. Hrenar, G. Rauhut, and H.-J. Werner. Impact of local and density fitting approximations on harmonic vibrational frequencies. J. Phys. Chem. A, 110:2060-2064, 2006.

[68] T. Hrenar, H.-J. Werner, and G. Rauhut. Accurate calculation of anharmonic vibrational frequencies of medium sized molecules using local coupled cluster methods. J. Chem. Phys., 126:134108, 2007.

[69] J. Pipek and P. G. Mezey. A fast intrinsic localization procedure applicable for ab initio and semiempirical linear combination of atomic orbital wave functions. J. Chem. Phys., 90:4916-4926, 1989.

[70] J. W. Boughton and P. Pulay. Comparison of the Boys and Pipek-Mezey localizations in the local correlation approach and automatic virtual basis selection. J. Comput. Chem., 14:736-740, 1993.

[71] H.-J. Werner and K. Pflüger. On the Selection of Domains and Orbital Pairs in Local Correlation Treatments. In D. C. Spellmeyer (editor), Annual Reports in Computational Chemistry, volume 2, pages 53-80. Elsevier, Amsterdam, Oxford, Boston, 2006.

[72] R. A. Mata and H.-J. Werner. Calculation of smooth potential energy surfaces using local electron correlation methods. J. Chem. Phys., 125:184110, 2006.

[73] R. A. Mata, H.-J. Werner, and M. Schütz. Correlation regions within a localized molecular orbital approach. J. Chem. Phys., 128:144106, 2008. 
[74] T. B. Adler and H.-J. Werner. An explicitly correlated local coupled cluster method for calculations of large molecules close to the basis set limit. J. Chem. Phys., 135:144117, 2011.

[75] M. Andrejić, U. Ryde, R. A. Mata, and P. Söderhjelm. Coupledcluster interaction energies for 200-atom host-guest systems. ChemPhysChem, 15:3270-3281, 2014.

[76] F. Neese. The ORCA program system. WIREs Comput. Mol. Sci., $2: 73-78,2012$.

[77] F. Neese, A. Hansen, and D. G. Liakos. Efficient and accurate approximations to the local coupled cluster singles doubles method using a truncated pair natural orbital basis. J. Chem. Phys., 131:64103, 2009.

[78] M. Schwilk, D. Usvyat, and H.-J. Werner. Communication: Improved pair approximations in local coupled-cluster methods. J. Chem. Phys., 142:121102, 2015.

[79] C. Riplinger, B. Sandhoefer, A. Hansen, and F. Neese. Natural triple excitations in local coupled cluster calculations with pair natural orbitals. J. Chem. Phys., 139:134101, 2013.

[80] P. Pinski, C. Riplinger, E. F. Valeev, and F. Neese. Sparse maps-A systematic infrastructure for reduced-scaling electronic structure methods. I. An efficient and simple linear scaling local MP2 method that uses an intermediate basis of pair natural orbitals. J. Chem. Phys., 143:34108, 2015.

[81] T. Kato. On the eigenfunctions of many-particle systems in quantum mechanics. Comm. Pure Appl. Math., 10:151-177, 1957.

[82] H.-J. Werner, G. Knizia, T. B. Adler, and O. Marchetti. Benchmark Studies for Explicitly Correlated Perturbation- and Coupled Cluster Theories. In F. M. Dolg (editor), Progress in Physical Chemistry Volume 3, pages 203-221. Oldenbourg Wissenschaftsverlag $\mathrm{GmbH}$, München, 2010.

[83] W. Kutzelnigg and W. Klopper. Wave functions with terms linear in the interelectronic coordinates to take care of the correlation cusp. I. General theory. J. Chem. Phys., 94:1985-2001, 1991.

[84] S. Ten-No. Initiation of explicitly correlated Slater-type geminal theory. Chem. Phys. Lett., 398:56-61, 2004. 
[85] E. F. Valeev. Improving on the resolution of the identity in linear R12 ab initio theories. Chem. Phys. Lett., 395:190-195, 2004.

[86] K. E. Yousaf and K. A. Peterson. Optimized auxiliary basis sets for explicitly correlated methods. J. Chem. Phys., 129:184108, 2008.

[87] K. A. Peterson, T. B. Adler, and H.-J. Werner. Systematically convergent basis sets for explicitly correlated wavefunctions: the atoms $\mathrm{H}$, He, B-Ne, and Al-Ar. J. Chem. Phys., 128:84102, 2008.

[88] T. B. Adler and H.-J. Werner. Local explicitly correlated coupledcluster methods: efficient removal of the basis set incompleteness and domain errors. J. Chem. Phys., 130:241101, 2009.

[89] T. B. Adler, G. Knizia, and H.-J. Werner. A simple and efficient CCSD(T)-F12 approximation. J. Chem. Phys., 127:221106, 2007.

[90] G. Knizia, T. B. Adler, and H.-J. Werner. Simplified CCSD(T)-F12 methods: theory and benchmarks. J. Chem. Phys., 130:54104, 2009.

[91] J. M. Bowman. The self-consistent-field approach to polyatomic vibrations. Acc. Chem. Res., 19:202-208, 1986.

[92] R. B. Gerber and J. R. Jung. The Vibrational Self-Consistent Field Approach and Extensions: Method and Applications to Spectroscopiy of Large Molecules and Clusters. In P. Jensen and P. R. Bunker (editors), Computational molecular spectroscopy, pages 365-390. Wiley, Chichester and New York, 2000.

[93] J. M. Bowman, S. Carter, and N. C. Handy. Progress in the quantum description of vibrational motion of polyatomic molecules. In C. E. Dykstra, G. Frenking, K. S. Kim, and G. E. Scuseria (editors), Theory and Applications of Computational Chemistry: The first forty years, pages 251-267. Elsevier, Amsterdam and Boston, 2005.

[94] O. Christiansen. Vibrational coupled cluster theory. J. Chem. Phys., 120:2149-2159, 2004.

[95] V. Barone. Anharmonic vibrational properties by a fully automated second-order perturbative approach. J. Chem. Phys., 122:14108, 2005.

[96] V. Barone, M. Biczysko, J. Bloino, M. Borkowska-Panek, I. Carnimeo, and P. Panek. Toward anharmonic computations of vibrational spectra for large molecular systems. Int. J. Quantum Chem., 112:2185-2200, 2012. 
[97] C. Adamo, A. J. Austin, V. Bakken, V. Barone, M. Bearpark, J. Bloino, E. Brothers, J. C. Burant, R. Cammi, M. Caricato, J. R. Cheeseman, J. Cioslowski, M. Cossi, J. B. Cross, A. D. Daniels, J. J. Dannenberg, S. Dapprich, M. Ehara, . Farkas, J. B. Foresman, D. J. Fox, M. J. Frisch, R. Fukuda, R. Gomperts, M. Hada, J. Hasegawa, J. J. Heyd, Y. Honda, H. P. Hratchian, M. Ishida, S. S. Iyengar, A. F. Izmaylov, J. Jaramillo, O. Kitao, M. Klene, J. E. Knox, R. Kobayashi, K. N. Kudin, X. Li, R. L. Martin, B. Mennucci, J. M. Millam, J. A. Montgomery, Jr., K. Morokuma, H. Nakai, T. Nakajima, H. Nakatsuji, J. Normand, J. W. Ochterski, F. Ogliaro, J. V. Ortiz, J. E. Peralta, G. A. Petersson, C. Pomelli, K. Raghavachari, N. Rega, A. Rendell, M. A. Robb, P. Salvador, G. Scalmani, H. B. Schlegel, G. E. Scuseria, J. L. Sonnenberg, V. N. Staroverov, R. E. Stratmann, J. Tomasi, K. Toyota, G. W. Trucks, G. A. Voth, T. Vreven, O. Yazyev, V. G. Zakrzewski, and G. Zheng. Gaussian 09 Revision D.01, Gaussian Inc. Wallingford CT 2009.

[98] H.-J. Werner, P. J. Knowles, G. Knizia, F. R. Manby, M. Schütz, P. Celani, T. Korona, R. Lindh, A. Mitrushenkov, G. Rauhut, K. R. Shamasundar, T. B. Adler, R. D. Amos, A. Bernhardsson, A. Berning, D. L. Cooper, M. J. O. Deegan, A. J. Dobbyn, F. Eckert, E. Goll, C. Hampel, A. Hesselmann, G. Hetzer, T. Hrenar, G. Jansen, C. Köppl, Y. Liu, A. W. Lloyd, R. A. Mata, A. J. May, S. J. McNicholas, W. Meyer, M. E. Mura, A. Nicklass, D. P. O’Neill, P. Palmieri, D. Peng, K. Pflüger, R. Pitzer, M. Reiher, T. Shiozaki, H. Stoll, A. J. Stone, R. Tarroni, T. Thorsteinsson, and M. Wang. MOLPRO, version 2012.1, a package of ab initio programs, Cardiff, UK, 2012.

[99] J. E. Del Bene. Proton affinities of ammonia, water, and hydrogen fluoride and their anions: a quest for the basis-set limit using the Dunning augmented correlation-consistent basis sets. J. Phys. Chem., 97:107-110, 1993.

[100] S. Grimme, J. Antony, S. Ehrlich, and H. Krieg. A consistent and accurate ab initio parametrization of density functional dispersion correction (DFT-D) for the 94 elements H-Pu. J. Chem. Phys., 132:154104, 2010.

[101] F. Weigend, A. Köhn, and C. Hättig. Efficient use of the correlation consistent basis sets in resolution of the identity MP2 calculations. $J$. Chem. Phys., 116:3175-3183, 2002. 
[102] Gaussian 09 Revision E.01 Release Notes. tt a ia $t$ t , accessed 16

Feb 2016. Specifically, the document states: "Frequencies using GD3BJ dispersion are now correct. Frequencies for other variants of Grimme D3 dispersion were correct in D.01 unless atoms having coordination numbers of 8 or more were involved".

[103] F. Kollipost, J. Andersen, D. W. Mahler, J. Heimdal, M. Heger, M. A. Suhm, and R. Wugt Larsen. The effect of hydrogen bonding on torsional dynamics: a combined far-infrared jet and matrix isolation study of methanol dimer. J. Chem. Phys., 141:174314, 2014.

[104] V. Dyczmons. Dimers of Ethanol. J. Phys. Chem. A, 108:2080-2086, 2004.

[105] C. Emmeluth, V. Dyczmons, T. Kinzel, P. Botschwina, M. A. Suhm, and M. Yáñez. Combined jet relaxation and quantum chemical study of the pairing preferences of ethanol. Phys. Chem. Chem. Phys., 7:991-997, 2005.

[106] F. Huisken, A. Kulcke, C. Laush, and J. M. Lisy. Dissociation of small methanol clusters after excitation of the $\mathrm{O}-\mathrm{H}$ stretch vibration at 2.7 u. J. Chem. Phys., 95:3924-3929, 1991.

[107] R. A. Provencal, J. B. Paul, K. Roth, C. Chapo, R. N. Casaes, R. J. Saykally, G. S. Tschumper, and H. F. Schaefer. Infrared cavity ringdown spectroscopy of methanol clusters: Single donor hydrogen bonding. J. Chem. Phys., 110:4258-4267, 1999.

[108] D. Rueda, O. V. Boyarkin, T. R. Rizzo, I. Mukhopadhyay, and D. S. Perry. Torsion-rotation analysis of $\mathrm{OH}$ stretch overtone-torsion combination bands in methanol. J. Chem. Phys., 116:91-100, 2002.

[109] J. S. Koehler and D. M. Dennison. Hindered Rotation in Methyl Alcohol. Phys. Rev., 57:1006-1021, 1940.

[110] J. Castillo-Chará and E. L. Sibert. Full dimensional theoretical study of the torsion-vibration eigenstates and torsional splittings of $\mathrm{CH}_{3} \mathrm{OH}$. J. Chem. Phys., 119:11671, 2003.

[111] D. Rueda, O. V. Boyarkin, T. R. Rizzo, A. Chirokolava, and D. S. Perry. Vibrational overtone spectroscopy of jet-cooled methanol from 5000 to $14000 \mathrm{~cm}^{-1}$. J. Chem. Phys., 122:44314, 2005. 
[112] I. Kleiner, G. T. Fraser, J. T. Hougen, and A. S. Pine. Molecular-beam optothermal spectrum of the $\mathrm{OH}$ stretching band of methanol. J. Mol. Spectrosc., 147:155-172, 1991.

[113] R. H. Hunt, W. N. Shelton, F. A. Flaherty, and W. B. Cook. Torsion-Rotation Energy Levels and the Hindering Potential Barrier for the Excited Vibrational State of the OH-Stretch Fundamental Band $v_{1}$ of Methanol. J. Mol. Spectrosc., 192:277-293, 1998.

[114] J. P. Perchard. The torsion-vibration spectrum of methanol trapped in neon matrix. Chem. Phys., 332:86-94, 2007.

[115] J. O. Henningsen. Improved molecular constants and empirical corrections for the torsional ground state of the $\mathrm{C}-\mathrm{O}$ stretch fundamental of $\mathrm{CH}_{3} \mathrm{OH}$. J. Mol. Spectrosc., 85:282-300, 1981.

[116] H. M. Pickett, E. A. Cohen, D. E. Brinza, and M. M. Schaefer. The submillimeter wavelength spectrum of methanol. J. Mol. Spectrosc., 89:542-547, 1981.

[117] G. Moruzzi, F. Strumia, J. C. S. Moraes, R. M. Lees, I. Mukhopadhyay, J. W. Johns, B. P. Winnewisser, and M. Winnewisser. The spectrum of $\mathrm{CH}_{3} \mathrm{OH}$ between 200 and $350 \mathrm{~cm}^{-1}$ : Torsional transitions and evidence for state mixings. J. Mol. Spectrosc., 153:511-577, 1992.

[118] O. V. Boyarkin, T. R. Rizzo, and D. S. Perry. Intramolecular energy transfer in highly vibrationally excited methanol. III. Rotational and torsional analysis. J. Chem. Phys., 110:11359, 1999.

[119] L. H. Xu and J. T. Hougen. Global Fit of Torsional-Rotational Transitions in the Ground and First Excited Torsional States of Methanol. J. Mol. Spectrosc., 173:540-551, 1995.

[120] M. E. Jacox. The spectroscopy of molecular reaction intermediates trapped in the solid rare gases. Chem. Soc. Rev., 31:108-115, 2002.

[121] J. P. Perchard, F. Romain, and Y. Bouteiller. Determination of vibrational parameters of methanol from matrix-isolation infrared spectroscopy and ab initio calculations. Part 1 - Spectral analysis in the domain 11000-200 $\mathrm{cm}^{-1}$. Chem. Phys., 343:35-46, 2008.

[122] L. Wan, L. Wu, A.-W. Liu, and S.-M. Hu. Neon matrix isolation spectroscopy of $\mathrm{CO}_{2}$ isotopologues. J. Mol. Spectrosc., 257:217-219, 2009. 
[123] E. E. Fileti, M. A. Castro, and S. Canuto. Calculations of vibrational frequencies, Raman activities and degrees of depolarization for complexes involving water, methanol and ethanol. Chem. Phys. Lett., 452:54-58, 2008.

[124] A. Bleiber and J. Sauer. The vibrational frequency of the donor $\mathrm{OH}$ group in the H-bonded dimers of water, methanol and silanol. Ab initio calculations including anharmonicities. Chem. Phys. Lett., 238:243$252,1995$.

[125] S. Grimme. Improved second-order Møller-Plesset perturbation theory by separate scaling of parallel- and antiparallel-spin pair correlation energies. J. Chem. Phys., 118:9095, 2003.

[126] R. F. Fink. Spin-component-scaled Møller-Plesset (SCS-MP) perturbation theory: a generalization of the MP approach with improved properties. J. Chem. Phys., 133:174113, 2010.

[127] K. E. Riley and P. Hobza. Assessment of the MP2 method, along with several basis sets, for the computation of interaction energies of biologically relevant hydrogen bonded and dispersion bound complexes. J. Phys. Chem. A, 111:8257-8263, 2007.

[128] R. A. Bachorz, F. A. Bischoff, S. Höfener, W. Klopper, P. Ottiger, R. Leist, J. A. Frey, and S. Leutwyler. Scope and limitations of the SCS-MP2 method for stacking and hydrogen bonding interactions. Phys. Chem. Chem. Phys., 10:2758-2766, 2008.

[129] R. N. Pribble, A. W. Garrett, K. Haber, and T. S. Zwier. Resonant ion-dip infrared spectroscopy of benzene- $\mathrm{H}_{2} \mathrm{O}$ and benzene-HOD. J. Chem. Phys., 103:531, 1995.

[130] R. N. Pribble, F. C. Hagemeister, and T. S. Zwier. Resonant ion-dip infrared spectroscopy of benzene-(methanol $)_{\mathrm{m}}$ clusters with $\mathrm{m}=1-6$. J. Chem. Phys., 106:2145, 1997.

[131] M. Mons, E. G. Robertson, and J. P. Simons. Intra- and Intermolecular $\pi$-Type Hydrogen Bonding in Aryl Alcohols: UV and IR-UV Ion Dip Spectroscopy. J. Phys. Chem. A, 104:1430-1437, 2000.

[132] A. Fujii, T. Ebata, and N. Mikami. An Infrared Study of $\pi$ Hydrogen Bonds in Micro-solvated Phenol: $\mathrm{OH}$ Stretching Vibrations of Phenol-X (X $=\mathrm{C}_{6} \mathrm{H}_{6}, \mathrm{C}_{2} \mathrm{H}_{4}$, and $\left.\mathrm{C}_{2} \mathrm{H}_{2}\right)$ Clusters in the Neutral and Cationic Ground States. J. Phys. Chem. A, 106:8554-8560, 2002. 
[133] E. C. Stanca-Kaposta, D. P. Gamblin, J. Screen, B. Liu, L. C. Snoek, B. G. Davis, and J. P. Simons. Carbohydrate molecular recognition: a spectroscopic investigation of carbohydrate-aromatic interactions. Phys. Chem. Chem. Phys., 9:4444-4451, 2007.

[134] A. W. Baker and A. T. Shulgin. Intramolecular Hydrogen Bonds to $\pi$-Electrons and Other Weakly Basic Groups. J. Am. Chem. Soc., 80:5358-5363, 1958.

[135] M. Tyblewski and A. Bauder. Microwave spectrum of bicyclo[2,2,1]hepta-2,5-dien-7-ol and spectroscopic evidence for an intramolecular $(\mathrm{OH} \cdots \pi)$ hydrogen bond. J. Mol. Struct., 102:267-277, 1983.

[136] P. Rademacher, L. Khelashvili, and K. Kowski. Spectroscopic and theoretical studies on intramolecular $\mathrm{OH}-\pi$ hydrogen bonding in 4substituted 2-allylphenols. Org. Biomol. Chem., 3:2620-2625, 2005.

[137] E. J. Ocola, A. A. Al-Saadi, C. Mlynek, H. Hopf, and J. Laane. Intramolecular $\pi$-type hydrogen bonding and conformations of 3 cyclopenten-1-ol. 2. Infrared and Raman spectral studies at high temperatures. J. Phys. Chem. A, 114:7457-7461, 2010.

[138] L. Andrews, G. L. Johnson, and B. J. Kelsall. Fourier transform infrared matrix spectra of substituted alkene-hydrogen fluoride complexes. J. Am. Chem. Soc., 104:6180-6186, 1982.

[139] J. A. Shea and W. H. Flygare. The rotational spectrum and molecular structure of the ethylene-HF complex. J. Chem. Phys., 76:4857-4864, 1982.

[140] K. G. Tokhadze and N. A. Tkhorzhevskaya. Infrared spectra of weak hydrogen-bonded complexes in cryogenic solutions. J. Mol. Struct., 270:351-368, 1992.

[141] L. H. Bjerkeseth, J. M. Bakke, and E. Uggerud. Inter- and intramolecular $\mathrm{O}-\mathrm{H} \cdots \pi$ hydrogen bonding in the methanol-ethene complex and syn-7-norbornenol, probed by IR, 1H NMR and quantum chemistry. $J$. Mol. Struct., 567-568:319-338, 2001.

[142] D. B. DuPré and M. C. Yappert. Cooperative Hydrogen- and $\pi \mathrm{H}-$ Bonded Interactions Involving Water and the Ethylenic Double Bond. J. Phys. Chem. A, 106:567-574, 2002. 
[143] E. Arunan, P. K. Mandal, M. Goswami, and B. Raghavendra. Rotational Spectra of Weakly Bound Complexes Containing $\mathrm{H}_{2} \mathrm{O} / \mathrm{H}_{2} \mathrm{~S}$ : Hydrogen Bonding vs van der Waals Interaction. Proc. Indian Natn. Sci. Acad. A, 71:377-389, 2005.

[144] A. Engdahl and B. Nelander. A matrix-isolation study of the ethylene-water interaction. Chem. Phys. Lett., 113:49-55, 1985.

[145] A. M. Andrews and R. L. Kuczkowski. Microwave spectra of $\mathrm{C}_{2} \mathrm{H}_{4} \cdot \mathrm{H}_{2} \mathrm{O}$ and isotopomers. J. Chem. Phys., 98:791, 1993.

[146] R. Medel, M. Heger, and M. A. Suhm. Molecular Docking via Olefinic $\mathrm{OH} \cdots \pi$ Interactions: A Bulky Alkene Model System and Its Cooperativity. J. Phys. Chem. A, 119:1723-1730, 2015.

[147] H. C. Gottschalk, J. Altnöder, M. Heger, and M. A. Suhm. Control over the Hydrogen-Bond Docking Site in Anisole by Ring Methylation. Angew. Chem. Int. Ed., 55:1921-1924, 2016.

[148] D. Zimmermann, T. Häber, H. Schaal, and M. A. Suhm. Hydrogen bonded rings, chains and lassos: The case of t-butyl alcohol clusters. Mol. Phys., 99:413-425, 2001. 

